\title{
AN INTOUATUE
}

\section{SOLATION DEMUE}

FOB ASEISWL DESIH

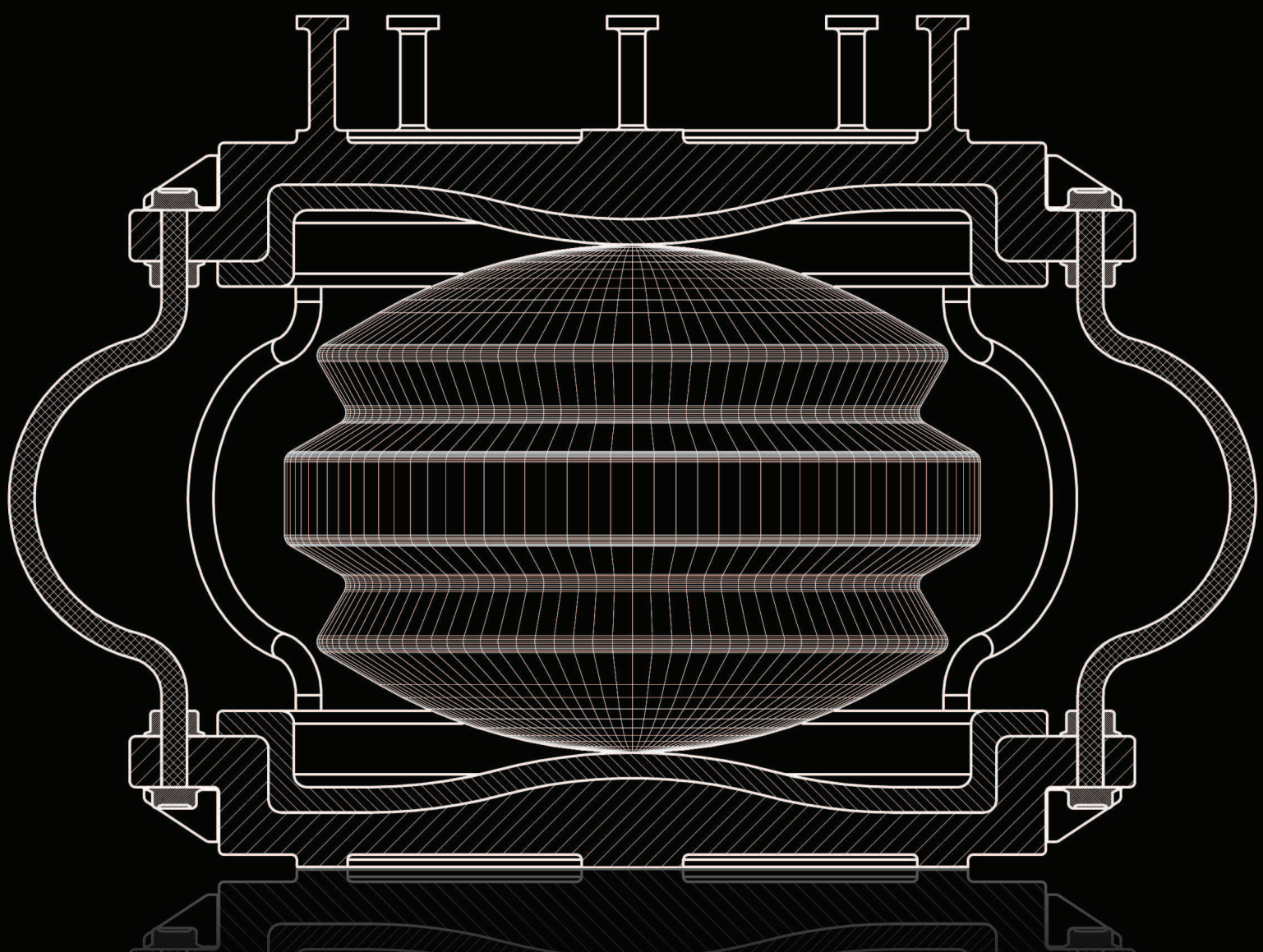

Mohammed Ismail Abdel-Kareem Moustafa

Doctoral Thesis

Barcelona, October 2009 
This page intentionally left blank. 


\section{(i:}

Technical University of Catalonia

School of Civil Engineering

Department of Applied Mathematics III

Control, Dynamics and Applications Group, CoDAlab

\section{Doctoral Thesis}

\section{An Innovative Isolation Device for Aseismic Design}

Doctoral Program:

Seismic Engineering and Structural Dynamics

Department of Soil Engineering, Mapping And

GEOPHYSICS

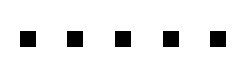

Author

Mohammed Ismail Abdel-Kareem Moustafa

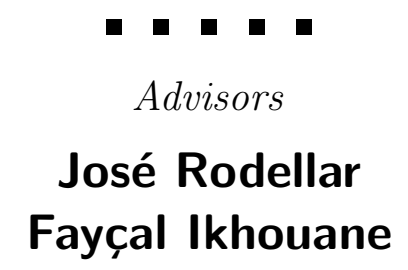

Barcelona September 27, 2009 



\section{(9:}

Universitat Politècnica de Catalunya

Escola Tècnica Superior d'Enginyers de Camins,

Canals i Ports de Barcelona

Departament de Matemàtica Aplicada III

Grup de Control, DinÀmica i Aplicacions, CoDAlab

\section{Tesi Doctoral}

\section{An Innovative Isolation Device for Aseismic Design}

Programa De Doctorat:

ENGinyeria Sísmica I Dinàmica Estructural

Departament D'Enginyeria Del Terreny, CARTogràficA I GEOFISICA

Autor

Mohammed Ismail Abdel-Kareem Moustafa

Asesors

José Rodellar

Fayçal Ikhouane

Barcelona September 27, 2009 



\section{Resumen}

Basado en la idea de reducir la demanda sísmica en lugar de aumentar la capacidad resistente de las estructuras, el aislamiento sísmico es un método simple para mitigar o reducir los posibles daños producidos por los terremotos. La correcta aplicación de esta tecnología conduce a un mejor comportamiento de las estructuras, que sigue siendo esencialmente elástico durante los terremotos de gran magnitud. El núcleo de esta tecnología es el aislador. La mayoría de los aisladores sísmicos disponibles en la actualidad siguen teniendo limitaciones prácticas que impiden que funcionen según lo previsto e imponen restricciones a su uso efectivo y al nivel de protección proporcionado.

En esta Tesis, se presenta un aislador sísmico avanzado llamado roll-n-cage (RNC). Se propone investigar su eficiencia a través de simulación numérica, en un intento de crear un sistema de aislamiento sísmico práctico, efectivo y económico, que tiene por objeto resolver los principales inconvenientes de los actuales sistemas de aislamiento sísmico, manteniendo sus principales ventajas. Este aislador incorpora aislamiento, disipación de energía, amortiguamiento y capacidad de fuerza recuperadora en una sola unidad. Además, ofrece una resistencia al viento significativa y una amplia gama de flexibilidad horizontal, por lo que es adecuado para proteger las estructuras de masa ligera, moderada y grande, así como para proteger equipos sensibles, hardware y / o antigüedades alojados en edificios. Por otra parte, las cuestiones relativas a la viabilidad, los costes de construcción y la disponibilidad de materiales, reducción o prevención de las respuestas de torsión y la resistencia a la elevación son abordados a fondo durante el diseño del aislador RNC.

El aislador RNC propuesto es descrito en profundidad y sus principios de funcionamiento son presentados en detalle. La caracterización mecánica del dispositivo se ha llevado a cabo por medio de un código computacional sofisticado que simula la respuesta de los dispositivos como si estuvieran sujetos a 
una máquina de pruebas reales. A través de este esquema, se consigue analizar numéricamente el comportamiento del aislador RNC bajo el efecto simultáneo de cargas horizontales y verticales, como se da típicamente en situaciones prácticas. Además, se presenta una descripción matemática de las principales características asociadas a la rodadura de los aisladores RNC. Asimismo se obtiene un modelo matemático para describir en una forma razonable y manejable la relación fuerza desplazamiento exhibida por el aislador de RNC.

Para evaluar la viabilidad del aislador RNC y para comprobar su capacidad para proteger los sistemas estructurales y no estructurales de los riesgos sísmicos, el dispositivo se implementa numéricamente en una variedad de estructuras con masas ligeras y grandes, además de en equipos sensibles alojados en los pisos superiores de dichas estructuras. Para extraer conclusiones de carácter relativamente general sobre el funcionamiento del aislador RNC, se estudia una amplia gama de terremotos y de características y propiedades de los aisladores y de las estructuras. Los resultados numéricos revelan que el aislador RNC propuesto puede reducir la respuesta sísmica frente a un amplio rango de excitaciones sísmicas, mientras que exhibe un rendimiento robusto para una gran variedad de estructuras.

La Tesis incluye como apéndice un estudio en profundidad sobre el modelo de histéresis de Bouc-Wen. El estudio contiene una revisión de los primeros y últimos avances y aplicaciones de este modelo, que es ampliamente utilizado en la descripción de fenómenos de histéresis en las estructuras. 


\section{Abstract}

Based on the concept of reducing seismic demand rather than increasing the earthquake resistant capacity of structures, seismic isolation is a surprisingly simple approach to mitigate or reduce earthquake damage potential. Proper application of this complex technology leads to better performing structures that will remain essentially elastic during large earthquakes. The core of this technology is the isolator. Most currently available seismic isolators still have practical limitations causing them not to function as anticipated and impose restrictions to their proper use and to the provided protection level.

In this dissertation, an advanced rolling-based seismic isolator, named rolln-cage (RNC) isolator, is proposed and investigated via numerical simulation as an attempt to create a practical, effective, and economic seismic isolation system that aims to fix the main drawbacks of the current seismic isolation systems while keeping their main advantages. This isolator incorporates isolation, energy dissipation, buffer and restoring force mechanisms in a single unit. Further, it offers a significant wind resistance and a great range of horizontal flexibility making it ideal to protect light, moderate and heavy mass structures as well as precious housed motion-sensitive equipment, hardware and/or antiquities. Moreover, issues related to practicality, construction costs and material availability, reducing or preventing torsional responses and uplift resistance are thoroughly addressed during the RNC bearing design.

The proposed RNC isolator is deeply described and its principles of operation are extensively highlighted. The mechanical characterization of the device has been carried out by means of a sophisticated computer code in a machine-like environment, which accurately simulates the response of the device subjected to a real testing machine. Through this machine-like environment, a general scheme is followed to numerically examine the behavior of the RNC isolator under simultaneous horizontal and vertical loads as in typical 
practical situations. Further, a mathematical description of the main features associated to rolling of the RNC isolator is presented. An input-output mathematical model is obtained to describe in a reasonable and manageable form the force-displacement relationship exhibited by the RNC isolator.

To assess the feasibility of the RNC isolator and to check its ability to protect structural and nonstructural systems from seismic hazards, it is numerically implemented to a variety of structures having light to heavy masses, in addition to motion-sensitive equipment housed in upper building floors. Further, and to draw relatively general conclusions about the performance of the $\mathrm{RNC}$ isolator, a wide range of ground motions, isolator characteristics and structural properties is considered. The numerical results reveal that the proposed RNC isolation bearing can mitigate the seismic responses under a variety of ground motion excitations while exhibiting robust performance for a wide range of structures.

The dissertation is appended with an in-depth survey, that contains a review of the past, recent developments and implementations of the versatile BoucWen model of smooth hysteresis, which is used extensively in modeling the hysteresis phenomenon in the dynamically excited nonlinear structures. This survey is the first of its kind about the model since its origination more than 30 years ago. The objective is to present some of the popular approaches that have utilized and/or developed that model to capture the hysteretic behavior offered by a variety of nonlinear systems. Then, the evaluation of their results and contributions (if any) is carried out to highlight their assets and limitations and to identify future directions in this research area. 


\section{Acknowledgements}

First and foremost, I would like to express my deepest thanks and gratitude to my advisors, Prof. José Rodellar and Prof. Fayçal Ikhouane for their support and guidance throughout the research. I am very grateful to the smiling professor José Rodellar for his tireless guidance, extreme support, insightful discussions and patient conduct. He has been very interested, active, open and smart. I would also like to extend my appreciation to Prof. Fayçal Ikhouane who smartly introduced me to the Bouc-Wen hysteretic model. I never forget their earnest and great efforts that enabled me to win a $\mathrm{PhD}$ grant and to study in the Technical University of Catalonia (UPC) in Barcelona, Spain.

My sincere appreciation is extended to all those who gave me the possibility to complete this thesis, especially the support of the Generalitat de Catalunya through the FI fellowship program by the Catalan Agency for Management of University and Research Grants (AGAUR). The support of the patent office in the Technical University of Catalonia (UPC) is also deeply appreciated.

I want to thank the Department of Applied Mathematics III and the Department of Soil Engineering, Mapping and Geophysics for giving me permission to commence this thesis in the Earthquake Engineering and Structural Dynamics doctoral program. I was also lucky to be able to associate myself with the talented and hard working members of the group of Control, Dynamics and Applications (CoDAlab) that never save effort nor support to help its members.

Finally, I would like to express my deepest appreciation to each member of my family. Especially I would like to give my special thanks to my wife. Without her unlimited encouragement, patience and love, since I knew her, I could never have a chance to finish my PhD in Spain. 
This page intentionally left blank. 


\section{Dedication}

To whom I owe my life, my parents.

To whom I always owe gratefulness, Samah, my wife.

To the eyes I have, Randa and Rana, my daughters.

To all brothers. 
This page intentionally left blank. 


\section{Contents}

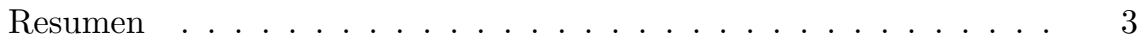

Abstract . . . . . . . . . . . ...... 5

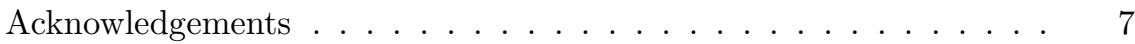

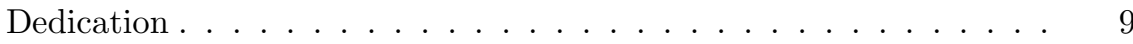

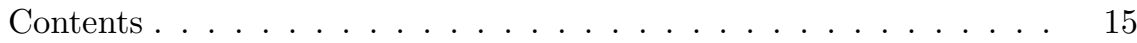

List of Tables . . . . . . . . . . . . . . . . . . . . . . . . 18

List of Figures . . . . . . . . . . . . . . . . . . . . . . . . . . . . . . . . . . . . . . . . 21

Nomenclature .................... 26

1 Introduction $\quad \mathbf{2 7}$

1.1 Motivations to seismic isolation . . . . . . . . . . . . . 27

1.2 Dissertation motivations . . . . . . . . . . . . . . . . . . . . . . . . . . 29

1.3 Dissertation objectives . . . . . . . . . . . . . . . . . . 30

1.4 Dissertation scope . . . . . . . . . . . . . . . . . . . . . . . . . . . . . . . . 32

1.5 Dissertation outline . . . . . . . . . . . . . . . . 32

2 Literature review $\quad 33$

2.1 Introduction . . . . . . . . . . . . . . . . 33

2.2 Philosophy behind seismic isolation . . . . . . . . . . . . . 33

2.2.1 Fundamental period perspective . . . . . . . . . . 34

2.2.2 Base shear perspective . . . . . . . . . . . . 36

2.2.3 Energy perspective . . . . . . . . . . . . . 37

2.3 Historical development of isolation systems . . . . . . . . . . . . 38

2.4 Seismic isolation devices . . . . . . . . . . . . . . 41

2.4.1 Elastomeric-based isolation systems . . . . . . . . . . 41

2.4.2 Sliding-based isolation systems . . . . . . . . . . . . 43

2.4.3 Spring-based isolation systems . . . . . . . . . . . . 47

2.4.4 Rolling-based isolation systems . . . . . . . . . . 47 
2.4.5 Synthetic liners and artificial soil layers . . . . . . . . 51

2.4.6 Rocking isolation system . . . . . . . . . . . . . 51

2.4.7 Sleeved-pile isolation system . . . . . . . . . . . . . 52

2.4.8 Other isolation systems . . . . . . . . . . 52

3 A new device for seismic isolation $\quad \mathbf{5 5}$

3.1 Motivations for a new isolator . . . . . . . . . . . 55

3.2 Concepts of the proposed isolator . . . . . . . . . . . 57

3.3 Technical field . . . . . . . . . . . . . . . . 58

3.4 Main forms ...................... . . . . 58

3.5 Main components . . . . . . . . . . . . . . . 59

3.6 Principles of operation . . . . . . . . . . . . . . 61

3.7 Component-mechanism relationship . . . . . . . . . . . 69

3.7.1 Rolling mechanism . . . . . . . . . . . . . . 69

3.7.2 Damping mechanism . . . . . . . . . . . . . 71

3.7.3 Bearing mechanism . . . . . . . . . . . . . . 72

3.7.4 Recentering mechanism . . . . . . . . . . . . 73

3.7.5 Buffer mechanism . . . . . . . . . . . . . . 74

3.7.6 Uplift-restraining mechanism . . . . . . . . . . . . 75

3.7.7 Initial stiffness mechanism . . . . . . . . . . . . . 75

3.8 Conclusions . . . . . . . . . . . . . . . . . 76

4 Modeling and characterization $\quad \mathbf{7 7}$

4.1 Introduction . . . . . . . . . . . . . . . . . . . . . 77

4.2 Mechanical characterization . . . . . . . . . . . . . . . . 78

4.2.1 Types of tests . . . . . . . . . . . . . . . 79

4.2 .2 Nonlinear modeling . . . . . . . . . . . . . . . 79

4.2.3 Mechanical characteristics . . . . . . . . . . . . . . 82

4.3 Mechanics of the elliptical rolling body . . . . . . . . . . . . 84

4.3.1 Motion of the elliptical rolling body . . . . . . . . . 85

4.3.2 Restoring mechanism of the elliptical rolling body . . . 87

4.4 Resistance to minor vibrations . . . . . . . . . . . . . . 89

4.5 Hysteretic modeling of the energy dissipation mechanism . . . . 92

4.5.1 The Bouc-Wen model . . . . . . . . . . . . . . 92

4.5.2 The standard Bouc-Wen model form . . . . . . . . . . 93

4.5.3 Parameters estimation of the standard form . . . . . . . 93

4.5.4 The normalized Bouc-Wen model form . . . . . . . . . . 93

4.5.5 Parameters estimation of the normalized form . . . . . . 94

4.5.6 Verification of the Bouc-Wen model . . . . . . . . . . . 94

4.6 Conclusions . . . . . . . . . . . . . . . . . . . . 94

5 Isolation of heavy mass buildings $\quad \mathbf{9 7}$

5.1 Introduction . . . . . . . . . . . . . . . . . . . 97

5.2 The used RNC isolator type . . . . . . . . . . . . . . . . . . . . . 97

5.3 Mechanical characteristics . . . . . . . . . . . . . . . . 98 
5.4 Hysteretic modeling . . . . . . . . . . . . . . . . . 99

5.4.1 Bouc-Wen model parameters estimation . . . . . . . . . 99

5.4.2 Seismic verification of the Bouc-Wen model . . . . . . . 102

5.5 Implementation . . . . . . . . . . . . . . . . . . . 103

5.5.1 Equations of motion . . . . . . . . . . . . . 104

5.5.2 Performance measures . . . . . . . . . . . . . 106

5.5 .3 Simulation tool . . . . . . . . . . . . . 106

5.5.4 Numerical study . . . . . . . . . . . . . . . . . . 107

5.6 Effect of isolator characteristics . . . . . . . . . . . . . 107

5.7 Recentering, damping and time response . . . . . . . . . . . . 109

5.8 Influence of superstructure flexibility . . . . . . . . . . . . . . 111

5.9 Behavior under long-period earthquakes . . . . . . . . . . . . . 114

5.10 Influence of earthquake characteristics . . . . . . . . . . . . . . 114

5.11 Conclusions . . . . . . . . . . . . . . . . . 117

6 Isolation of light-to-moderate mass buildings $\quad \mathbf{1 2 1}$

6.1 Introduction . . . . . . . . . . . . . . . . . . 121

6.2 The used RNC isolator type . . . . . . . . . . . . . . . . . 122

6.3 Mechanical characteristics . . . . . . . . . . . . . . 122

6.4 Hysteretic modeling . . . . . . . . . . . . . . . . 123

6.4.1 Bouc-Wen model parameters estimation . . . . . . . . 123

6.4.2 Seismic verification of the Bouc-Wen model . . . . . . . 124

6.5 Implementation . . . . . . . . . . . . . . . . . . . . . . . . . 125

6.5.1 Equations of motion . . . . . . . . . . . . 126

6.5.2 Performance measures . . . . . . . . . . . . . . . 127

6.5.3 Simulation tool . . . . . . . . . . . . . . 128

6.5.4 Numerical study . . . . . . . . . . . . . . . . . . 128

6.6 Frequency response analysis . . . . . . . . . . . . . . . . . . . . . 128

6.7 Time history analysis . . . . . . . . . . . . . . . . . . . 129

6.8 Influence of superstructure flexibility . . . . . . . . . . . . . 130

6.9 Influence of isolator and earthquake characteristics . . . . . . . 132

6.10 Conclusions . . . . . . . . . . . . . . . 135

7 Equipment isolation: isolated raised floor approach $\quad 141$

7.1 Introduction . . . . . . . . . . . . . . . . . . . . . 141

7.2 The used RNC isolator type . . . . . . . . . . . . . . . . . 143

7.3 Mechanical characteristics . . . . . . . . . . . . . . . . . 143

7.4 Hysteretic modeling . . . . . . . . . . . . . . . . . . . . . 143

7.4.1 Bouc-Wen model parameters estimation . . . . . . . . . 144

7.4.2 Seismic verification of the Bouc-Wen model . . . . . . . 144

7.5 Implementation . . . . . . . . . . . . . . . . . . . . . . 145

7.5.1 Equations of motion . . . . . . . . . . . . . . 148

7.5.2 Performance measures . . . . . . . . . . . . . . . 149

7.5.3 Simulation tool . . . . . . . . . . . . . . . . . . 149

7.5.4 Displacement demand against isolator height . . . . . . 149 
7.6 Numerical assessment . . . . . . . . . . . . . . . . . . 150

7.6.1 Actual earthquakes . . . . . . . . . . . . 151

7.6.2 Harmonic excitations . . . . . . . . . . . . . . 155

7.7 Conclusions . . . . . . . . . . . . . . . 155

8 Equipment isolation: isolated structure approach $\quad \mathbf{1 6 3}$

8.1 Introduction . . . . . . . . . . . . . . . . . 163

8.2 The used RNC isolator type . . . . . . . . . . . . . . . . . 164

8.3 Mechanical characteristics . . . . . . . . . . . . . . . . 165

8.4 Hysteretic modeling . . . . . . . . . . . . . . . . 165

8.4.1 Bouc-Wen model parameters estimation . . . . . . . . . 166

8.4.2 Seismic verification of the Bouc-Wen model . . . . . . 166

8.5 Implementation . . . . . . . . . . . . . . . . . . . . 167

8.5.1 Equations of motion . . . . . . . . . . . . 169

8.5.2 Structure equipment interaction . . . . . . . . . 170

8.5.3 Performance measures . . . . . . . . . . . . . . . 172

8.5.4 Simulation tool ................ . . . . 173

8.6 Response of structure-equipment systems . . . . . . . . . . . 173

8.6.1 Harmonic excitations . . . . . . . . . . . . . . . . . 175

8.6.2 Actual earthquakes . . . . . . . . . . . . . 177

8.7 Conclusions . . . . . . . . . . . . . . . . . . . . 181

9 Conclusions and future work $\quad \mathbf{1 8 5}$

9.1 Contributions . . . . . . . . . . . . . . . . 185

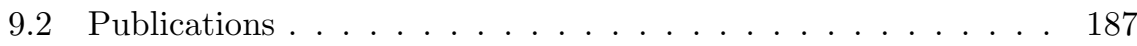

9.2.1 Patents ..................... 187

9.2 .2 Journal papers . . . . . . . . . . . . . . . . . . . . 187

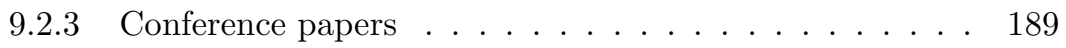

9.3 Future work . . . . . . . . . . . . . . . . 189

$\begin{array}{lr}\text { A The Bouc-Wen model, a survey } & 191\end{array}$

A.1 Introduction . . . . . . . . . . . . . . . . . . . . . . 191

A.2 Physical and mathematical consistency of the model . . . . . . 194

A.2.1 Physical consistency of the Bouc-Wen model . . . . . . 195

A.2.2 Mathematical consistency of the Bouc-Wen model . . . 200

A.3 Description of the hysteresis loop . . . . . . . . . . . . . . . 201

A.4 Variation of hysteresis loop with model parameters . . . . . . . 204

A.4.1 Parameters-loop relationship, analytical study . . . . . . 205

A.4.2 Parameters-loop relationship, numerical study . . . . . 206

A.5 Interpretation of the model parameters . . . . . . . . . . . . 208

A.5.1 Interpretation of the standard model parameters . . . . 208

A.5.2 Interpretation of the normalized model parameters . . . 209

A.6 The Bouc-Wen model parameter identification . . . . . . . . . 209

A.6.1 Least-squares based identification . . . . . . . . . . . . 209

A.6.2 Kalman filter based identification . . . . . . . . . . . 212 
A.6.3 Genetic algorithm based identification . . . . . . . . . . 214

A.6.4 Gauss-Newton iterative based identification . . . . . . . 216

A.6.5 Bootstrap filter based identification . . . . . . . . . . . 217

A.6.6 Identification using periodic signals . . . . . . . . . . . . 217

A.6.7 Simplex method based identification . . . . . . . . . . 220

A.6.8 Support vector regression based identification . . . . . . 220

A.6.9 Constrained nonlinear optimization based identification 220

A.6.10 Non-parametric identification . . . . . . . . . . . . 221

A.7 Modeling using the Bouc-Wen model . . . . . . . . . . . . . . . 221

A.7.1 Magnetorheological dampers . . . . . . . . . . . 221

A.7.2 Structural elements . . . . . . . . . . . . . . . . 224

A.7.3 Base isolation devices . . . . . . . . . . . . . . . . . 225

A.7.4 Mechanical systems . . . . . . . . . . . . . 226

A.7.5 Piezoelectric actuators . . . . . . . . . . . . 227

A.7.6 Soil behavior . . . . . . . . . . . . . . . . . 227

A.7.7 Energy dissipation systems . . . . . . . . . . . . . 228

A.8 Conclusion . . . . . . . . . . . . . . . . . . 229

B Used softwares $\quad 231$

$\begin{array}{ll}\text { Bibliography } & 232\end{array}$

$\begin{array}{lr}\text { Index } & 266\end{array}$ 
This page intentionally left blank. 


\section{List of Tables}

3.1 Isolation devices advantages and disadvantages . . . . . . . 56

4.1 Contact pairs properties of the modeled RNC isolator . . . . . 82

5.1 Characteristics of different RNC-a and RNC-b isolator sets. . . 99

5.2 Identified model parameters for RNC-a and RNC-b isolators . 100

5.3 Discrepancy between measured and predicted outputs . . . . . 104

5.4 Modal properties of example structures . . . . . . . . . . . . . 118

5.5 Influence of earthquake intensity on RNC-a isolator . . . . . . 119

5.6 Influence of earthquake frequency contents on RNC-a isolator . 119

5.7 Results summary using 36 earthquakes, RNC-a and RNC-b . . 120

6.1 Different $\mathrm{RNC}-\mathrm{c}$ isolator design sets . . . . . . . . . . . . . . . 123

6.2 Modal properties of fixed-base and isolated light structures . . 127

6.3 Effect of superstructure flexibility, isolation set II . . . . . . . . 136

6.4 Effects of isolator properties and earthquake intensity, set I . . 137

6.5 Effects of isolator properties and earthquake intensity, set II . . 138

6.6 Effects of isolator properties and earthquake intensity, set III . 139

6.7 Results summary under 36 earthquakes, RNC-c . . . . . . . . . 140

7.1 Modal properties of example structures without equipment . . 157

7.2 Response summary under 36 seismic excitations . . . . . . . . . 158

7.3 Displacement demands against the RNC isolator heights . . . . 159

7.4 Harmonic excitation frequency vs equipment responses, $\frac{1}{2} K_{\mathrm{s}}$. . 160

7.5 Harmonic excitation frequency vs equipment responses, $K_{\mathrm{s}} \quad$. . 161

7.6 Harmonic excitation frequency vs equipment responses, $2 K_{\mathrm{s}}$. . 162 
8.1 Characteristics of different $\mathrm{RNC}-\mathrm{c}$ isolator sets . . . . . . . . . 165

8.2 Modal properties of housing structures without equipment . . . 182

8.3 Influence of structural period and equipment tuning . . . . . . 183

8.4 Response summary under 36 seismic excitations . . . . . . . . . 184

A.1 Classification of BIBO stable Bouc-Wen models . . . . . . . . . 196 A.2 Maximal hysteretic output vs the normalized model parameters 207 A.3 Hysteretic zero $\bar{x}^{\circ}$ vs the normalized model parameters . . . . . 207

A.4 Hysteretic output $\bar{w}(\bar{x})$ vs the normalized model parameters . . 230 


\section{List of Figures}

2.1 Single story base-isolated structure . . . . . . . . . . . . 35

2.2 Acceleration and deformation spectra . . . . . . . . . . 37

3.1 RNC isolator for light to moderate mass systems type c, $\mathrm{RNC}-\mathrm{c} \quad 61$

3.2 RNC isolator for heavy mass systems type a, RNC-a . . . . . . 62

3.3 RNC isolator for heavy mass systems type b, RNC-b . . . . . . 63

3.4 Unidirectional RNC isolator, type c . . . . . . . . . . . . 64

3.5 Details of the RNC type c, RNC-c . . . . . . . . . . . . . 65

3.6 Details of the RNC type a, RNC-a . . . . . . . . . . . . 66

3.7 Details of the RNC type b, RNC-b . . . . . . . . . . . . . 67

3.8 Connection between metallic dampers and bearing plates . . . 68

3.9 Rolling, damping and zero uplift mechanisms . . . . . . . . . . 69

3.10 Buffer mechanism . . . . . . . . . . . . . . . . . . . 70

3.11 Gravity-based recentering mechanism . . . . . . . . . . . . 71

3.12 The main design bearing area of RNC-a and RNC-b . . . . . . 72

4.1 ANSYS model, non-deformed shape, RNC-c . . . . . . . . . . 83

4.2 ANSYS model, non-deformed + deformed shapes, RNC-c . . . 84

4.3 ANSYS model, non-deformed shape, RNC-a . . . . . . . . 85

4.4 ANSYS model, non-deformed + deformed shapes, RNC-a . . . 86

4.5 ANSYS model, non-deformed shape, RNC-b . . . . . . . . . . 87

4.6 ANSYS model, non-deformed + deformed shapes, RNC-b . . . 88

4.7 ANSYS measured response and its bilinear approximation . . . 89

4.8 Mechanics of the elliptical rolling body . . . . . . . . . . . . . . 90

4.9 Free-body-diagram of the elliptical rolling body . . . . . . . . . 91

5.1 Actual ANSYS output vs predicted Bouc-Wen model output . 101 
5.2 Simplified ANSYS output, bilinear and Bouc-Wen model outputs 101

5.3 Actual ANSYS output force and its bilinear approximation . . 102

5.4 Bouc-Wen model validation for RNC-a and RNC-b isolators . 103

5.5 Case-study heavy-mass structure isolated by the RNC-a isolator 105

5.6 Frequency response of different designs of RNC-a isolator . . . 108

5.7 Frequency response of different designs of RNC-b isolator . . . 109

5.8 Harmonic response time history, RNC type b, set II . . . . . . 110

5.9 Time history of top floor acceleration under Kobe earthquake . 111

5.10 Time history of bearing displacement under Kobe earthquake . 112

5.11 Accel. and disp. response spectra under three earthquakes . . . 113

5.12 Response spectra under long-period Mexico City earthquake . . 115

5.13 Normalized Fourier amplitude of Kobe, Northridge and Parkfield 116

6.1 Measured vs calculated restoring force for RNC-c . . . . . . . . 124

6.2 Model validation for $\mathrm{RNC}-\mathrm{c}$ in light building isolation . . . . . 125

6.3 Case study light mass building structure . . . . . . . . . . . . . 126

6.4 Frequency response of different designs of $\mathrm{RNC}-\mathrm{c}$ isolator . . . 130

6.5 Acceleration time histories using different $\mathrm{RNC}-\mathrm{c}$ isolator sets . 131

6.6 Behavior of RNC-c isolator under a long-period earthquake . . 134

7.1 Measured vs calculated restoring of the reduced scale $\mathrm{RNC}-\mathrm{c}$. 144

7.2 Model validation for the reduced scale $\mathrm{RNC}-\mathrm{c}$ isolator . . . . . 145

7.3 Idealized building, raised-floor and equipment model . . . . . . 146

7.4 Cases of study . . . . . . . . . . . . . . . . . 147

7.5 Acceleration response spectra for the ground and the 7 th floors 150

7.6 Absolute acceleration time history, El-Centro earthquake . . . . 152

7.7 Relative displacement time history, El-Centro earthquake . . . 153

7.8 Absolute acceleration time history, Mexico-city earthquake . . . 154

7.9 Isolated vs non-isolated equipment response . . . . . . . . . . . 154

8.1 Measured vs calculated restoring force, harmonic excitation . . 166

8.2 Measured vs calculated restoring force, seismic excitation . . . 167

8.3 Idealized structure-equipment system . . . . . . . . . . . . . . 168

8.4 Model of an isolated structure-equipment system . . . . . . . . 170

8.5 Acceleration response spectra for ground and 7 th floors . . . . 174

8.6 Structural fundamental frequency vs equipment acceleration . . 175

8.7 Harmonic equipment displacement time history . . . . . . . . . 176

8.8 Harmonic acceleration time history of equipment . . . . . . . . 177

8.9 Acceleration time history of equipment . . . . . . . . . . . . . 178

8.10 Equipment acceleration time history, long-period earthquake . 180

A.1 Graph force versus displacement for a hysteresis function . . . 192

A.2 Evolution of the Bouc-Wen model literature . . . . . . . . . . . 194

A.3 The $T$-periodic input signals . . . . . . . . . . . . . . . . . . . . 202

A.4 Methodologies of the analysis of the variation of $\bar{w}(\bar{x}) \quad \ldots$. . . 206 
a The larger radius of an ellipse

$b \quad$ The smaller radius of an ellipse

$A \quad$ A parameter of Bouc-Wen model

$A_{\text {loop }} \quad$ The hysteresis loop area

$c_{01}$ A material constant that characterizes its deviatoric deformation

$c_{10} \quad$ A material constant that characterizes its deviatoric deformation

$c_{1} \quad$ The damping of the first floor

$c_{\text {eff }} \quad$ The effective damping coefficient

$c_{\mathrm{e}} \quad$ The damping of equipment

$k_{\text {eff }} \quad$ The effective damping coefficient

C The damping matrix

$\mathbf{C}_{\mathrm{s}} \quad$ The damping matrix of the superstructure

$C_{1} \quad$ The base-isolated natural frequency of vibration coefficient or yield stress

$C_{2} \quad$ The base-isolated natural period of vibration coefficient or tangent modulus

d The material incompressibility parameter

$d_{\max }$ The peak positive isolator shear displacement 
$d_{\text {min }} \quad$ The peak negative isolator shear displacement

D The yield displacement

$e \quad$ The base of natural logarithm or eccentricity of an ellipse

E The energy quantity

$E_{\mathrm{sb}} \quad$ The strain energy of the isolator

$E_{\mathrm{sp}} \quad$ The strain energy potential

$E_{\mathrm{ss}} \quad$ The strain energy of the structure

$E_{\mathrm{s}} \quad$ The total strain energy of the system (isolator plus structure)

$f \quad$ The structural natural frequency

$f_{\mathrm{e}} \quad$ The frequency ratio of excitation to equipment

$f_{\mathrm{s}} \quad$ The frequency ratio of excitation to structure

$F_{\mathrm{b}} \quad$ The hysteretic restoring force of Bouc-Wen model

$F_{\mathrm{LR}} \quad$ The limiting lateral force due to rolling

$F_{\mathrm{LY}} \quad$ The limiting lateral force due to yielding

$F_{\mathrm{L}} \quad$ The total limiting lateral force

$F_{\max }$ The peak positive isolator shear force

$F_{\text {min }} \quad$ The peak negative isolator shear force

$g \quad$ The ground acceleration

$x_{\mathrm{g}} \quad$ The ground displacement

$i \quad$ The complex numbers notation

$\bar{I}_{1} \quad$ The first deviatoric strain invariant

$\bar{I}_{2} \quad$ The second deviatoric strain invariant

$J_{\mathrm{r}} \quad$ The rotational moment of inertia

$k \quad$ A parameter of the Bouc-Wen model

$k_{1} \quad$ The stiffness of the first floor

$k_{\mathrm{b}} \quad$ The isolator post-yield stiffness

$k_{\text {eff }} \quad$ The effective stiffness of a bearing 
$k_{\mathrm{e}} \quad$ The stiffness of equipment or elastic isolator stiffness

K The stiffness matrix

$\mathbf{K}_{\mathrm{s}} \quad$ The stiffness matrix of the superstructure

$K \quad$ The initial bulk modulus

$m_{\mathrm{b}} \quad$ The base mass

$m_{\mathrm{r}} \quad$ The raised-floor mass

M The mass matrix

$\mathbf{M}_{\mathrm{s}} \quad$ The mass matrix of the superstructure

$M_{\mathrm{r}} \quad$ The recentering couple

$n \quad$ A parameter of Bouc-Wen model

$p \quad$ Half a vertical distance

$P_{\text {des }} \quad$ The design vertical load

Q The isolator characteristic strength

$\mathbf{r} \quad$ The vector of influence coefficients

$S_{\mathrm{a}} \quad$ The spectral absolute acceleration

$S_{\mathrm{d}} \quad$ The spectral relative displacement

$t \quad$ The time

$T \quad$ The duration, or vibration period

$T_{\mathrm{nb}} \quad$ The base-isolated natural vibration period

$T_{\mathrm{n}} \quad$ The natural vibration period

$V_{\max } \quad$ The maximum base shear

$w \quad$ An auxiliary variable of Bouc-Wen model

W The structural weight

$\ddot{x}_{\mathrm{b}} \quad$ The base mass acceleration

$\ddot{x}_{\mathrm{e}} \quad$ The relative acceleration at the CG of equipment

$\ddot{x}_{\mathrm{f}} \quad$ The mounting floor acceleration

$\ddot{x}_{\mathrm{g}} \quad$ The ground acceleration 
$\ddot{x}_{\mathrm{r}} \quad$ The raised-floor acceleration

$\dot{x}_{\mathrm{e}} \quad$ The relative velocity at the CG of equipment

$\ddot{\mathbf{x}}_{\mathrm{S}} \quad$ The relative acceleration vector of superstructure

$\dot{\mathbf{x}}_{\mathrm{s}} \quad$ The relative velocity vector of superstructure

$\mathbf{x}_{\mathrm{S}} \quad$ The relative displacement vector of superstructure

$x_{1} \quad$ The base mass relative displacement

$x_{2} \quad$ The main mass relative displacement

$x_{\mathrm{b}} \quad$ The base displacement

$x_{\mathrm{e}} \quad$ The relative displacement at the CG of equipment

$x_{\mathrm{r}} \quad$ The horizontal displacement of the rolling body

$X_{\mathrm{g}} \quad$ The amplitude of harmonic ground excitation

$y_{1} \quad$ The base mass total displacement

$y_{2} \quad$ The main mass total displacement

$y_{\mathrm{r}} \quad$ The vertical displacement of the rolling body

$z \quad$ An auxiliary variable of Bouc-Wen model

$\beta_{\text {eff }} \quad$ The effective damping ratio of a bearing

$\eta \quad$ The number of isolators

$\beta \quad$ A parameter of Bouc-Wen model

$\gamma \quad$ A parameter of Bouc-Wen model

$\kappa_{x} \quad$ A parameter of Bouc-Wen model

$\kappa_{w} \quad$ A parameter of Bouc-Wen model

$\mu \quad$ The initial shear modulus, or friction coefficient

$\nu \quad$ The poisson ratio

$\omega \quad$ The circular frequency in $\mathrm{rad} / \mathrm{sec}$

$\omega_{\mathrm{nb}} \quad$ The circular base-isolated natural frequency in $\mathrm{rad} / \mathrm{sec}$

$\omega_{\mathrm{n}} \quad$ The circular natural frequency in $\mathrm{rad} / \mathrm{sec}$

$\rho \quad$ A parameter of Bouc-Wen model 
$\sigma \quad$ A parameter of Bouc-Wen model

$\theta \quad$ The eccentric angle of an ellipse

$\theta_{\mathrm{r}} \quad$ The rotation of the elliptical body

$\varepsilon \quad$ The relative error

$\varepsilon_{\mathrm{b}} \quad$ The mass ratio of base to structure

$\varepsilon_{\mathrm{e}} \quad$ The mass ratio of equipment to structure

$\xi \quad$ The damping ratio

1 Subscript that refers to the $L_{1}$ norm, or first floor, or fundamental mode of vibration

$\infty \quad$ Subscript that refers to the $L_{\infty}$ norm

b Subscript that refers to the base mass, or the Bouc-Wen model restoring force

e Subscript that refers to the equipment, or the excitation

f Subscript that refers to the floor

g Subscript that refers to the ground

i Subscript that refers to the variable number, or the input energy

$\mathrm{k} \quad$ Subscript that refers to the kinetic energy

m Subscript that refers to the measured restoring force from ANSYS

$\mathrm{N} \quad$ Subscript that refers to the number of degrees of freedom

p Subscript that refers to the potential energy

r Subscript that refers to the raised floor

s Subscript that refers to the structure, or the strain energy

y Subscript that refers to the metallic yield damper

$\xi \quad$ Subscript that refers to the structural damping

ANSYS ANSYS finite element computer code

BIBO Bounded input bounded output

CG Center of gravity

DE Dissipated energy 
DOF Degree of freedom

EDF Electric de France

EERC Earthquake engineering research center

FKN Contact stiffness factor

FKT Default tangent stiffness factor

FNA Fast nonlinear analysis

FP Friction pendulum

FPS Friction pendulum system

FTOLN Penetration tolerance factor

HDR High damping rubber bearing

HE Hysteretic energy

IE Input energy

KE Kinetic energy

LRB Lead rubber bearing

MFPS Multiple friction pendulum system

MU Maximum initial friction coefficient

PGA Peak ground acceleration of an earthquake

PINB Default pinball region factor

R-FBI Resilient-friction base isolation base isolation

RNC-a Roll-n-cage isolator, type a

RNC-b Roll-n-cage isolator, type b

$\mathrm{RNC}-\mathrm{c}$ Roll-n-cage isolator, type c

RNC Roll-n-cage isolator

SCF Sliding concave foundation

SE Strain energy

SLTOL Default elastic slip factor

TAUMAX Maximum friction stress

VE Viscous energy

max Special function of maximum value

sign Special function of maximum value 


\section{Introduction}

\subsection{Motivations to seismic isolation}

Earthquakes are potentially devastating natural events which threaten lives, destroy property, and disrupt life-sustaining services and societal functions. Hence, to ensure human safety and comfort, it is necessary to mitigate earthquake hazards in vulnerable communities through investigating how buildings and their nonstructural components, lifelines, and highway structures behave and are affected by earthquakes, how damage to these structures impacts society, and how these damages can be attenuated through innovative means.

Conventionally, seismic design of building structures is based on the concept of increasing the resistance capacity of the structures against earthquakes by employing, for example, the use of shear walls, braced frames, or momentresistant frames. However, these traditional methods often result in high floor accelerations for stiff buildings, or large interstory drifts for flexible buildings. Because of this, the building contents and nonstructural components may suffer significant damage during a major earthquake, even if the structure itself remains basically undamaged. This is not tolerable for buildings whose contents are more costly and valuable than the buildings themselves. High-precision production factories are one example of buildings that contain extremely costly and sensitive equipment. Additionally, hospitals, police and fire stations, and telecommunication centers are examples of facilities that contain valuable equipment and should remain operational immediately after an earthquake.

In order to minimize interstory drifts and to reduce floor accelerations, the concept of seismic isolation is increasingly being adopted as a nonconventional design approach of structures. Seismic isolation is a practical design strategy 
that has been used for seismic rehabilitation of existing buildings and in the design of a number of new buildings. The effect of seismic isolation can be achieved through installation of certain devices between the building and the supporting foundation, so as to separate or isolate the motion of the building from that of the ground making them basically uncoupled. The applicability of the concept of seismic isolation need not be restricted to the structure in its entirety. It can be applied as well to the isolation of sensitive equipment mounted inside a building from undesired floor vibrations through, for example, installation of an isolation system between the equipment base and the supporting floor.

Conceptually, isolation reduces the superstructure response by separating the structure from damaging seismic ground motions. Typical isolation systems reduce seismic forces transmitted to the superstructure by lengthening the period of the building and adding some amount of damping. Added damping is an inherent property of most isolators, but may also be provided by supplemental energy dissipation devices installed across the isolation interface. Under favorable conditions, the isolation system reduces drift in the superstructure by a factor of at least two and sometimes by as much as factor of five from that which would occur if the building were not isolated. Floor accelerations are also reduced in the structure, although the amount of reduction depends on the force-deflection characteristics of the isolators and may not be as significant as the reduction of drift. Reduction of drift in the superstructure protects structural components and elements as well as nonstructural components sensitive to drift-induced damage. Reduction of acceleration protects nonstructural components that are sensitive to acceleration-induced damage such as motion-sensitive equipment, machinery and hardware.

Another nonconventional design approach to improving earthquake response performance and damage control is that of supplemental energy dissipation systems. In these systems, mechanical devices are incorporated into the frame of the structure to dissipate energy throughout the height of the structure. In addition to increasing the energy dissipation capacity of a structure, some energy dissipation systems also increase the strength and stiffness. Such systems include: metallic-yielding, friction, and viscoelastic. Energy dissipation systems utilizing fluid viscous dampers will not generally increase the strength or stiffness of a structure unless the excitation frequency is high. In general, the addition of an energy dissipation system will result in a reduction in drift, therefore, reduction of damage, but this is accompanied with an increase in the total lateral force exerted on the structure due to increased strength and/or stiffness.

It is not possible with conventional structural systems nor supplemental energy dissipation systems to reduce simultaneously both inter-story drift and floor accelerations. However, the dynamics of seismic isolation allow the designer to do just that. Further, seismic isolation allows the reflection of a great 
deal of the total lateral force (base shear) transmitted into the structure from the ground due to earthquake shocks, which can not be attained using energy dissipation systems.

Seismic isolation and supplemental energy dissipation systems belong to the so called passive control systems, as the control of structural motions is not exercised through a logically driven external force, but rather through a specially designed interface at the structural base or within the structure. In contrast, the techniques of active structural control, which are still under research and development for the seismic resistance of structures, require the installation of some logically controlled external systems, such as actuators, to counteract the structural motions. One drawback with active control techniques is the relatively high cost of maintenance for the control system and actuators, which should remain functional at all times in order to respond to a major earthquake. There also exists another categories of techniques, called semiactive and hybrid control, that attempt to make use of the best of both passive control and active control devices.

\subsection{Dissertation motivations}

Seismic isolation is a mature technology that has been proven to be an effective means for protecting structures and attached equipment. There are a number of acceptable isolation systems, the construction of which is well understood. Nevertheless, the concept appears to have an irresistible attraction to inventors, and many new and different systems of isolators are proposed and patented each year. Many of these new systems will prove to be impractical and some might actually be lethal, but the number continues to increase year by year.

The existing passive seismic isolation systems can be categorized into four main groups [401, 333]: (1) elastic, (2) rolling-based, (3) elastomeric, and (4) sliding-based bearings. The elastic bearings provide adequate horizontal flexibility, but it is accompanied with vertical flexibility. This generates vertical acceleration component out from the horizontal acceleration. Further, they lack damping and efficient re-centering mechanisms. The rolling-based bearings provide incomparable horizontal flexibility, but they require damping, recentering mechanisms and sufficient bearing capacity for heavy mass structures. Moreover, they offer no wind resistance. The elastomeric bearings offer great vertical stiffness, for heavy masses, associated with sufficient horizontal flexibility. However, some design limitations must be fulfilled to avoid buckling or $p-\Delta$ failure of the bearing, which imposes restrictions on their use for a variety of structures especially those of light masses. Furthermore, an isolated structure by elastomeric bearings is susceptible to torsion under earthquakes. On the other hand, a sliding-based bearing, named the friction pendulum system (FPS), could overcome the majority of drawbacks stated before, but unfor- 
tunately on account of structure uplift, changeable coefficient of friction and fixed vibration period, which represents a severe practical difficulty for aseismic design [331].

The above isolation systems are based on well known and accepted physical principles, but no device is perfect as they are still having some functional drawbacks. This urges the efforts either to enhance the existing devices or to innovate others with the aim of attaining the maximum protection level of structural and nonstructural elements through seismic isolation. Unfortunately, most of the isolation systems reported in the literature are patented products. The same is also true with most newly invented products. Therefore, not all of them are readily available for procurement and direct enhancement. Thus, the intention may be directed toward creating more efficient isolation devices.

\subsection{Dissertation objectives}

So far, no one has solved the problems associated with ideal isolation systems that necessitate full structure-ground horizontal separation with no negative effects. This dissertation attempts to develop a novel practical and more efficient isolation system than the current ones. Such isolation system is referred to as roll-n-cage (RNC) isolator. It integrates, in a single unit, the best of the present day isolation devices, while discarding their main drawbacks. Briefly, the proposed isolator is designed to possess unique properties for a seismic isolator, including:

1. Multi- and unidirectional isolation.

2. Energy dissipation.

3. Uplift restraint.

4. Resistance to wind and minor vibrations.

5. Built-in buffer.

6. Inherent gravity-based recentering mechanism.

7. Suitable for light, moderate and heavy mass systems.

8. Resistance to flattening of contact surfaces.

9. Wide range of stiffness and damping.

10. Independent damping and bearing mechanisms.

11. Independent stiffness and bearing mechanisms.

12. Great system-base decoupling.

13. Non-fixed vibration period.

14. Expected reasonable construction cost.

Then, the dissertation follows a carefully designed strategy that allows full description, mechanical characterization, mathematical modeling and numerical assessment of the proposed isolator. Such strategy may be summarized in the following specific objectives: 
- Introducing the proposed isolation device highlighting the following points:

- Concepts of the proposed isolator.

- Technical field.

- Main forms.

- Main components.

- Principles of operation.

- Component-mechanism relationship.

- Modeling and characterization of the proposed isolation device through:

- Mechanical characterization, using a commercial finite-element computer code, in a machine-like environment, which accurately simulates the response of the device subjected to a real testing machine.

- Analytical description of the mechanics of the elliptical rolling body.

- Estimation of the resistance to minor vibrations.

- Hysteretic modeling of the energy dissipation mechanism.

- Verification of the obtained hysteretic model.

- Providing a design tool for the rolling body geometry.

- Application of the proposed isolation device to a variety of structural and nonstructural systems for the purpose of efficiency assessment. This includes:

- Isolation of heavy-mass buildings structures.

- Isolation of light- to moderate-mass buildings structures.

- Isolation of motion-sensitive equipment using two approaches:

1. Isolated raised-floor in a fixed-base housing structure.

2. Isolated housing structure equipped with fixed-base equipment.

- Performing thorough numerical studies using time history analysis, response spectrum analysis and frequency response analysis, which aim at a comprehensive assessment of the proposed isolator. These studies include:

- Influence of isolator characteristics.

- Evaluation of self-recentering mechanism and added damping.

- Influence of the superstructure flexibility.

- Behavior under long-period earthquakes.

- Influence of earthquake characteristics.

- Structure-equipment interaction and influence of different structureequipment tuning conditions. 


\subsection{Dissertation scope}

As far as the scope is concerned, only linear building structures and their inner motion-sensitive equipment are adopted for the numerical evaluation of the proposed isolation device efficiency. Only a single horizontal component of the earthquake ground motion is considered at a time. The effects of soilstructure interaction is not considered in this work. The most important response quantities, from structural engineering point-of-view, are chosen as performance measures to represent the comparison reference between base-isolated and fixed-base conditions. Such performance measures are:

- Structural absolute accelerations at the topmost point.

- Base or bearing relative-to-ground displacements.

- Story drift.

- Base shear.

- Equipment absolute accelerations.

- Equipment relative-to-floor displacement, only when the isolated raised floor approach is adopted.

\subsection{Dissertation outline}

This dissertation has been structured as follows. In Chapter 1, the problem of seismic-resistant structural design is presented. Motivations to seismic isolation are highlighted. The dissertation motivations and objectives are briefly stated. Chapter 2 introduces the philosophy behind seismic isolation systems and their historical development. Chapter 3 motivates the need to innovate an isolation bearing and presents a novel rolling-based isolation bearing named roll-n-cage (RNC) isolator. A detailed description of the RNC isolator is presented and its principles of operation are explained. Mechanical characterization and mathematical modeling of the RNC isolator are given in Chapter 4. The RNC isolator is numerically implemented to heavy mass building structures in Chapter 5 , and to light-to-moderate mass buildings in Chapter 6. The motion-sensitive equipment are protected from seismic hazards by means of the RNC isolator using two different approaches: the isolated raised floor housed in a fixed-base structure in Chapter 7 and the isolated housing structure that is equipped with fixed-base equipment in Chapter 8. The main research conclusions and recommendations for future work are summarized in Chapter 9. Further, all the different publications taken from this dissertation are also listed. In Appendix A, an extensive survey about the versatile Bouc-wen model of smooth hysteresis is presented. All the papers that the author used and is aware of have been cited in the Bibliography. 


\section{Literature review}

\section{$2.1 \quad$ Introduction}

Seismic isolation is a design strategy based on the premise that it is both possible and feasible to uncouple a structure from the ground and thereby protect it from the damaging effects of the earthquake motions. to achieve this result, while at the same time satisfying all of the in-service functional requirements, additional flexibility is introduced usually at the base of the structure. Additional damping is also provided so as to control the deflections which occur across the isolation interface.

\subsection{Philosophy behind seismic isolation}

Decoupling the structure from the horizontal components of the ground motion gives the structure a fundamental frequency that is much lower than its fixedbase frequency and the predominant frequencies of the ground motion. The first dynamic mode of the isolated structure involves deformation only in the isolation system, the structure above being to all intents and purposes rigid. The higher modes that produce deformation in the structure are orthogonal to the first mode, and consequently, to the ground motion. These higher modes do not participate in motion, so that the high energy in the ground motion at these higher frequencies can not be transmitted to the structure. The isolation system does not absorb the earthquake energy, but rather deflects it through the dynamics of the system; this effect does not depend on damping, but a certain level of damping is beneficial to suppress possible resonance at the isolation frequency. 


\subsubsection{Fundamental period perspective}

Insight into the benefits of using base isolators in structures can be gained by considering the special case of a single-storey linear undamped structure, which is separated from the ground by flexible bearings of lateral linear stiffness $k_{\mathrm{b}}$ as shown in Fig. 2.1(a). The bearings are connected together through a (base mass) rigid horizontal diaphragm of mass $m_{\mathrm{b}}$, which lies just above the bearings. The whole system is idealized as a 2DOFs spring-mass system as shown in Fig. 2.1(b). The governing equations of motion are

$$
\begin{aligned}
& m_{\mathrm{b}} \ddot{y}_{1}+k\left(y_{1}-y_{2}\right)+k_{\mathrm{b}}\left(y_{1}-x_{\mathrm{g}}\right)=0 \\
& m \ddot{y}_{2}+k\left(y_{2}-y_{1}\right)=0
\end{aligned}
$$

where $m$ is the main mass, $k$ is the stiffness of the structure above the isolator and $y_{1}$ and $y_{2}$ are the total displacements of the base and the main masses, respectively.

If the relative displacements between the masses and the supports are defined to be

$$
\begin{aligned}
& x_{1}=y_{1}-x_{\mathrm{g}} \\
& x_{2}=y_{2}-x_{\mathrm{g}}
\end{aligned}
$$

it then follows from substituting (2.2) into (2.1) that

$$
\begin{gathered}
m_{\mathrm{b}} \ddot{x}_{1}-k x_{2}+\left(k+k_{\mathrm{b}}\right) x_{1}=-m_{\mathrm{b}} \ddot{x}_{\mathrm{g}} \\
m \ddot{x}_{2}+k x_{2}-k x_{1}=-m \ddot{x}_{\mathrm{g}}
\end{gathered}
$$

Consider the special case where $m_{\mathrm{b}}$ is very small and so is assumed to be zero. Therefore $(2.3 \mathrm{a})$ becomes

$$
-k x_{2}+\left(k+k_{\mathrm{b}}\right) x_{1}=0
$$

Solving for $x_{1}$ in terms of $x_{2}$ in (2.4) gives

$$
x_{1}=\left(\frac{k}{k+k_{\mathrm{b}}}\right) x_{2}=\left(\frac{1}{1+\left(k_{\mathrm{b}} / k\right)}\right) x_{2}
$$

The displacement $x_{1}$ is the displacement of the base isolator relative to the ground. Equation (2.5) gives the value of $x_{1}$ in terms of $x_{2}$ and the ratio of the stiffness of the isolator to the structure. Note that if $k_{\mathrm{b}}$ goes toward infinity (i.e. very stiff bearing), then $x_{1}$ goes toward zero. Also, if $k_{\mathrm{b}}$ is equal $k$, then $x_{1}$ is equal to one half of $x_{2}$. The ideal, or perfect, isolation case is attained if $k_{\mathrm{b}}$ goes toward zero. In this case, $x_{1}=x_{2}$ which translates into zero story drift, perfect rigid-body vibration of the structure and full structure-ground separation in the horizontal direction. 
a

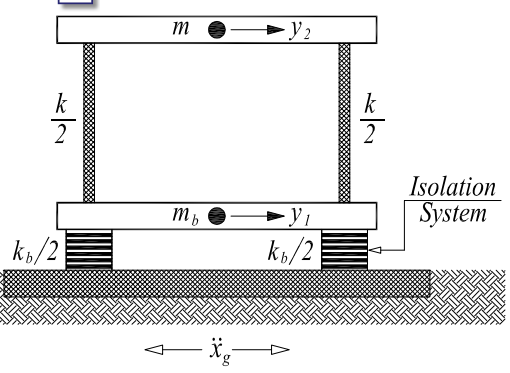

b

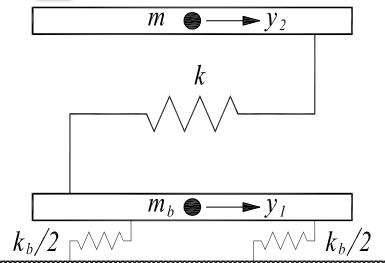

Figure 2.1: Single story base-isolated structure

Substituting (2.5) into (2.3b) gives the equation of motion for this springmass system as

$$
m \ddot{x}_{2}+\left[1-\left(\frac{1}{1+\left(k_{\mathrm{b}} / k\right)}\right)\right] k x_{2}=-m \ddot{x}_{\mathrm{g}}
$$

One very important effect of the presence of base isolators, seen in (2.6), is the modification of the natural frequency of vibration of the system. In this spring-mass system example, the natural frequency of vibration is

$$
\omega_{\mathrm{nb}}=\sqrt{\frac{k}{m}\left[1-\left(\frac{1}{1+\left(k_{\mathrm{b}} / k\right)}\right)\right]}=C_{1} \omega_{\mathrm{n}}
$$

where $\omega_{\mathrm{n}}=\sqrt{k / m}$, and $C_{1}$ is the base isolated natural frequency of vibration coefficient, defined as

$$
C_{1}=\sqrt{1-\left(\frac{1}{1+\left(k_{\mathrm{b}} / k\right)}\right)}
$$

The natural period of vibration is

$$
T_{\mathrm{nb}}=\frac{2 \pi}{\omega_{\mathrm{nb}}}=\frac{2 \pi}{\sqrt{\frac{k}{m}\left[1-\left(\frac{1}{1+\left(k_{\mathrm{b}} / k\right)}\right)\right]}}=C_{2} T_{\mathrm{n}}
$$

where $T_{\mathrm{n}}=2 \pi / \omega_{\mathrm{n}}$, and

$$
C_{2}=\frac{1}{\sqrt{1-\left(\frac{1}{1+\left(k_{\mathrm{b}} / k\right)}\right)}}=\frac{1}{C_{1}}
$$

Insight into the meaning of a rigid, or fixed, base structure can be gained from (2.7). If $k_{\mathrm{b}}$ is much greater than $k_{1}$, then the term in the denominator, 
that is, $1+\left(k_{\mathrm{b}} / k\right)$, of $(2.7)$ becomes large, and therefore $\omega_{\mathrm{nb}}$ approach the natural frequency of a rigid base system $\sqrt{k / m}$ and $T_{\mathrm{nb}}$ approaches the natural period of vibration of a rigid base system $2 \pi / \sqrt{k / m}$.

The situation of interest for a base isolated structure is the case where $k_{\mathrm{b}}$ is less than $k$. In the limit if $k_{\mathrm{b}}$ is very small, then $\omega_{\text {nb }}$ goes to zero, see (2.7), and the natural period of vibration of the structure $T_{\mathrm{nb}}$ goes to infinity, see (2.9) which corresponds to the fully isolated condition. Equation (2.5) can be rewritten to express the ratio $x_{1} / x_{2}$ as a function of the ratio $k_{\mathrm{b}} / k$. In this case, if $k_{\mathrm{b}} / k$ becomes large, then $x_{1} / x_{2}$ tends to zero. This is the fixed base condition.

\subsubsection{Base shear perspective}

The response of the spring-mass system, shown in Fig. 2.1, to an earthquake ground motion can be obtained using the response spectra analysis. It follows from Eq. (2.6) that the maximum relative displacement, $\left(x_{1}\right)_{\max }$, and maximum base shear, $V_{\max }$, are equal to:

$$
\begin{aligned}
\left(x_{1}\right)_{\max } & =S_{\mathrm{d}}\left(T_{\mathrm{nb}}, \zeta\right)=\left(T_{\mathrm{nb}} / 2 \pi\right)^{2} S_{a}\left(T_{\mathrm{nb}} \zeta\right) \\
V_{\max } & =m S_{\mathrm{a}}\left(T_{\mathrm{nb}}, \zeta\right)
\end{aligned}
$$

in which $S_{\mathrm{d}}$ is the spectral displacement and $S_{\mathrm{a}}$ is the pseudo spectral acceleration.

The pseudo acceleration response spectra, as shown schematically in Fig. $2.2(\mathrm{a})$, represent plots of the peak value of $S_{\mathrm{a}}$ with respect to the fundamental natural period of vibration $T_{\mathrm{nb}}$ of the structure, which can be obtained as a by-product of the deformation response spectra shown in Fig. 2.2(b) through the use of the relation in Eq. (2.11).

From Eq. (2.9), smaller value of $k_{\mathrm{b}}$ increases the value of $T_{\mathrm{nb}}$. Then, two important features can be observed from the response spectra given in Figs. $2.2(\mathrm{a})$ and 2.2(b). The first is the so-called period shift effect. As indicated by Fig. 2.2(a) and Eq. (2.12), substantial reduction in the pseudo acceleration or the base shear of a structure is possible, if the period of vibration of the structure is significantly lengthened through installation of base isolators. In general, the additional flexibility needed to lengthen the period of the structure will give rise to large relative displacements across the isolators, as indicated by Fig. 2.2(b). The second is the so-called energy dissipating effect. If additional damping is introduced into the structure, then the deformation of the structure can be significantly reduced (see Fig. 2.2(b)). Also, it can be seen that a smaller base shear force will be induced on a structure having larger damping (see Fig. 2.2(a)), and that a structure responds less sensitively to variations in ground motion characteristics, as indicated by the smoother response curves for structures with higher damping levels in both figures. 

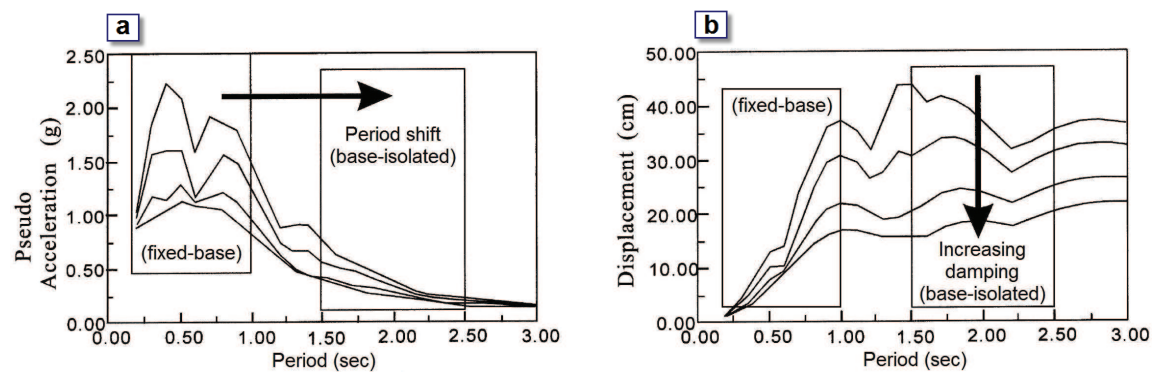

Figure 2.2: (a) Pseudo-acceleration spectra, (b) Deformation spectra

As revealed by the aforementioned two seismic response spectra, the philosophy behind the installation of base isolators is to lengthen the period of vibration of the protected structure, so as to reduce the base shear induced by the earthquake, while providing additional damping or reducing the relative displacements across the isolators themselves.

\subsubsection{Energy perspective}

When a structure is subjected to a strong earthquake, the system energy of the structure can be conceptually expressed as:

$$
K E+D E+S E=I E
$$

where $K E$ denotes the kinetic energy; $D E$ the dissipated energy, which equals the sum of $V E$ and $H E$, with $V E$ denoting the viscous energy and $H E$ the hysteretic energy; $S E$ is the strain energy and $I E$ the seismic input energy.

In Eq. (2.13), $K E$ and $S E$ are the portion of the energy of the structure that is recoverable, whereas $V E$ and $H E$ are the portion that is dissipative. For a fixed-base building structure, when $I E$ is not so large, the energy input to the structure will be dissipated in the form of $V E$. When a strong earthquake occurs, if all the input energy cannot be dissipated by the viscous damping of the structure, then the residual energy will be dissipated in the form of $H E$. If the structure has been designed to have sufficient ductility, then it may undergo plastic deformations in certain joints, members or specially added components, but the phenomenon of collapse must be avoided. This is the ductility concept of design for the traditional fixed-base structures.

The response of a base-isolated structural system can be viewed from a strain energy perspective. Consider the spring $k_{b}$ to be the base isolation substructure and $k$ to be the superstructure above the base isolator. The total strain energy in the sub- and superstructure is the total strain energy in the 
whole structure and can be written as

$$
\begin{aligned}
E_{\mathrm{sb}} & =\frac{1}{2} k_{\mathrm{b}} x_{1}^{2} \\
E_{\mathrm{ss}} & =\frac{1}{2} k\left(x_{2}-x_{1}\right)^{2}
\end{aligned}
$$

where $E_{\mathrm{sb}}$ is the strain energy in spring $k_{\mathrm{b}}$ (i.e. the base isolator) and $E_{\mathrm{ss}}$ is the strain energy in spring $k$ (i.e. the superstructure). The strain energy in the superstructure is a function of $\left(x_{2}-x_{1}\right)$. Therefore, the total strain energy of the system, $E_{\mathrm{s}}$ (i.e. base isolator plus superstructure), is

$$
E_{\mathrm{s}}=E_{\mathrm{sb}}+E_{\mathrm{ss}}=\frac{1}{2} k_{\mathrm{b}} x_{1}^{2}+\frac{1}{2} k\left(x_{2}-x_{1}\right)^{2}
$$

The difference in displacements between $x_{2}$ and $x_{1}$ can be written using (2.5), and it follows that

$$
x_{2}-x_{1}=\left(\frac{k+k_{\mathrm{b}}}{k}\right) x_{1}-x_{1}=\left(\frac{k_{\mathrm{b}}}{k}\right) x_{1}
$$

Therefore, substituting (2.17) into (2.15), the strain energy in the superstructure above the isolator is equal to

$$
E_{\mathrm{ss}}=\frac{1}{2} k\left(\frac{k_{\mathrm{b}}}{k}\right)^{2} x_{1}^{2}=\frac{1}{2} k_{\mathrm{b}}\left(\frac{k_{\mathrm{b}}}{k}\right) x_{1}^{2}
$$

It then follows from $(2.16)$ that

$$
E_{\mathrm{s}}=E_{\mathrm{sb}}+E_{\mathrm{ss}}=\frac{1}{2} k_{\mathrm{b}} x_{1}^{2}+\frac{1}{2} k_{\mathrm{b}}\left(\frac{k_{\mathrm{b}}}{k}\right) x_{1}^{2}
$$

Equations (2.14), (2.15), (2.18), and (2.19) show that as $k_{\mathrm{b}}$ decreases in magnitude, the strain energy in the $k_{\mathrm{b}}$ spring (base isolator substructure) increases in relation to the strain energy in the $k$ spring (superstructure). Therefore, introducing base isolators redirects the strain energy from the superstructure above the base isolators into the base isolators themselves.

\subsection{Historical development of isolation systems}

Seismic isolation is not a very new idea. More than a century ago, in 1885 , John Milne, a professor of engineering in Japan, built a small wooden house on balls in cast-iron plates with saucer-like edges on the heads of piles, to demonstrate that a structure could be isolated from earthquake shaking [181]. However, the building behavior under wind loads was not satisfactory. So, he reduced the balls diameter from 10 inch to $1 / 4$ inch. By this mean, the 
building became stable against wind loads and was evidently successful under actual earthquake action. In 1891, after Narobi earthquake, a Japanese person, Kawai, proposed a base isolated structure with timber logs placed in several layers in the longitudinal and transverse direction [220].

In 1906, Jacob Bechtold of Germany applied for a U.S. patent in which a seismic-resistant building is to be placed on rigid plate supported on spherical bodies of hard material [59]. In 1909, a medical doctor from England, Calentarients, had submitted a patent application to the British patent office for a method of building construction. In his method, a building is constructed on a layer of fine sand, mica, or talc that would allow the building to slide in an earthquake, thereby reducing the force transmitted to the building itself [243].

In 1929, Robert de Montalk of New Zealand filed a patent application for an invention comprising a means whereby a bed is placed and retained between the base of a building and its solid foundation. The bed was being composed of material which will absorb or minimize seismic shocks [116].

There are almost a hundred known proposals for a seismic isolation systems made prior to 1960, but as far as can be determined, none were ever built. The most probable reason is a lack of practicality and the fact that the engineering profession of the day had little or no confidence in their success [59]. One notable historetic structure, however, is Frank Lloyd Wright's Imperial Hotel in Tokyo, completed in 1921. This building was founded on a shallow layer of firm soil which in turn was supported by an underlying layer of mud. Cushioned from devastating ground motion, the hotel survived the 1923 Tokyo earthquake and later Wright wrote in his autography [465] of the "merciful provision" of 60 to 70 feet of soft mud below the upper 8 foot thick surface soil layer which supported the building. The imperial Hotel is evidence that base isolation works and seismic protection can be achieved by relatively simple means.

Since the 1920s there have been other "accidents" in which some structures have survived earthquakes while neighboring buildings have collapsed. Several unreinforced masonry buildings were only lightly damaged in the 1933 Long Beach earthquake because they were able to slide on their grade beams. At least one masonry house survived the 1976 Tangshan earthquake because it also slid on its foundation inadvertently. Reconnaissance reports also describe instances of slender structures surviving earthquakes because of their ability to rock or sidesway. Water tanks and statues, chandeliers and suspension bridges are some examples.

Attempts were made in the 1930s to protect the upper floors of multistory buildings by designing very flexible first-story columns. It was proposed that the first-story columns should be designed to yield during an earthquake to produce isolation and energy-absorbing actions. However, to produce enough damping, several inches of displacements is required, and a yielded column has greatly buckling loads, proving the concept to be impractical. To prevent the structure from moving too far, the first story is constructed underground and 
energy dissipators are installed at the top of this story [23]. To overcome the inherent dangers of soft supports at the base, many types of roller bearing systems have been proposed. The rollers and spherical bearings are very low in damping and have no inherent resistance to lateral loads, and therefore some other mechanisms that provide wind restraint and energy absorbing capacity are needed. A long duration between two successive earthquakes may result in the cold welding of bearings and plates, thus causing the system to become rigid after a time. Therefore, the application of the rolling supports was restricted to the isolation of special components of low or moderate weight [73].

Parallel to the development of the soft first-story approach, the flexibility of natural rubber was also seen to be another solution for increasing the flexibility of the system. The first use of a rubber isolation system to protect a structure from earthquakes was in 1969 for a three-story elementary school in Skopje, Republic of Macedonia. The building was constructed of reinforced concrete shear walls and supported by 54 large blocks of hard rubber. These rubber blocks were completely unreinforced, so the weight of the building causes them to bulge sideways. To improve the building stability under minor vibrations, glass blocks acting as seismic fuzes are intended to break when the seismic loading exceeds a certain threshold. Owing to having the same stiffness of the isolation system in all directions, the building bounces and rocks backwards and forwards [234]. These types of bearings are unsuitable for the earthquake protection of structures.

The subsequent development of laminated rubber bearings in 1970s has made seismic isolation a practical reality [374, 375, 433]. These bearings are very stiff in the vertical direction to carry the structural weight but are very flexible horizontally to enable the isolated structure to move laterally under strong ground motion. In the early 1980s, developments in rubber technology led to new rubber compounds which were termed high damping rubber (HDR) [117]. Later, a large number of isolation devices were developed including rollers, springs, friction slip plates, capable suspension, sleeved piles, and rocking foundations. Now seismic isolation has reached the stage of gaining acceptance and replacing the conventional construction, at least for important structures.

It is not just the invention of the elastomeric bearing which has made seismic isolation a practical reality. Three other parallel, but independent, developments have also contributed to its success. The first of these was the development of reliable software for the computer analysis of structures so as to predict their performance and determine design parameters. The second development was the use of shaking tables which are able to simulate the effects of real recorded earthquake ground motions on different types of structures. A third important development is in the skill of the engineering seismologist in estimating ground motions at a particular site. 


\subsection{Seismic isolation devices}

By now seismic isolation is used in many countries, and there are a number of acceptable isolation systems, whose mechanisms and characteristics are well understood. Nevertheless, the concept is irresistable to inventors, and many new and different systems or isolators are proposed and patented each year. Many of these new systems have been proven impractical and some might actually be lethal, but the number continues to increase year by year [333].

The successful seismic isolation of a particular structure is strongly dependent on the appropriate choice of the isolation system. In addition to providing adequate horizontal flexibility and appropriate damping, the isolation system should essentially have the capability of self-centering after deformation, high vertical stiffness to avoid rocking, and enough initial stiffness to avoid frequent vibration from wind and minor seismic events. Different types of isolators have been developed and proposed to achieve these properties, and some of them are discussed below.

\subsubsection{Elastomeric-based isolation systems}

Rubber bearings offer a simple method of seismic isolation and are relatively easy to manufacture. The bearings are made by vulcanization bonding of successive rubber and steel reinforcing thin sheets [245]. The bearings are very stiff in the vertical direction and flexible in the horizontal direction. High vertical stiffness is achieved through the laminated construction of the bearing using steel plates, where the horizontal flexibility is caused by rubber layers. The common elastomers used in elastomeric bearings are natural rubber, neoprene rubber, butyl rubber and nitrile rubber. The natural rubber is the most frequently recommended material because its main mechanical characteristics are superior to those of synthetic elastomers [422]. Laminated elastomeric bearings are available in three main classes: low-damping rubber bearings, high-damping rubber bearings and lead-plug rubber bearings.

\section{Low-damping rubber bearings}

In these bearings, the damping ratio provided by the elastomer is low, in the order of 2 to $4 \%$, and therefore they are unusual to be used without some other elements that provide suitable damping. The rubber material behavior in shear is quite linear up to shear strains above $100 \%$. The low-damping rubber bearings are simple to manufacture, easy to model and their mechanical response is unaffected by rate, temperature, or aging [333]. 


\section{Lead-plug rubber bearings}

These bearings were invented in New Zealand in 1975 [374, 375] and are similar to the laminated rubber bearings, but a central lead core is used to provide an additional means of energy dissipation. The energy absorbing capacity by the lead core reduces the lateral displacements of the isolator. The lead plug produces a substantial increase in damping from about $3 \%$ of critical damping in the natural rubber to about $15 \%$, and also increases the resistance to minor earthquakes or wind. The steel plates force the lead plug to deform in shear. Generally, the lead yields at a relatively low stress of about $10 \mathrm{MPa}$ in shear and behaves approximately as an elasto-plastic solid. The interrelated simultaneous process of recovery, recrystallization and grain growth is continuously restoring the mechanical properties of the lead. The lead has good fatigue properties during cyclic loading at plastic strains and is also readily available at high purity of $99.9 \%$ required for its predictable mechanical properties. The leadplug bearings behave essentially as hysteretic damper device and were widely studied by $[249,252,400]$.

\section{High-damping rubber bearings}

In the early 1980s, developments in rubber technology lead to new rubber compounds, which were termed "high damping rubber". These compounds produced bearings that had a high stiffness at low shear strains but a reduced stiffness at high strain levels. These bearings were originally developed in England in 1982 to eliminate the need for supplementary damping elements. The damping is increased to levels between 10 and $20 \%$ at $100 \%$ shear strains [333, 253].

\section{Other elastomeric bearings}

The use of steel shims in laminated rubber bearings provides necessary vertical stiffness, but at the same time makes these bearings heavy and expensive. In 2001, [247] proposed an economic seismic isolation system for developing country. In this system, the steel plates are replaced by fiber mesh. The fiberreinforced elastomeric bearing is expected to be significantly lighter and could be easier to be manufactured.

A Japanese rubber company has developed an elastomeric bearing that combines a standard low-damping rubber bearing with a large internal hole, into which a central plug of a very high-damping synthetic elastomer is placed. The diameter of the internal plug is about half that of the whole bearing. The shear modulus of the two elastomers is chosen to be very close. The effective damping of this combined isolation system is about 18 to $20 \%$ [53]. 


\subsubsection{Sliding-based isolation systems}

In this class of isolation systems, the superstructure is allowed to slide during major seismic events. The structure slides whenever the lateral force exceeds the friction force at the sliding interface. The horizontal friction force at the sliding surface offers resistance to minor seismic events or wind and dissipates energy.

\section{Pure sliding systems}

Purely sliding systems are the earliest and simplest isolation systems to be proposed. These systems have no inherent natural period and therefore are insensitive to variations in the frequency content of ground excitations. The acceleration at the base of the structure is limited to the coefficient of friction at the sliding interface. Therefore, by keeping this coefficient of friction low, the acceleration transmitted to the structure can be reduced. However, the friction coefficient cannot be reduced arbitrarily, as the sliding displacement may exceed acceptable values. The main drawbacks of the these systems are the absence of restoring force, cold welding, freezing, deterioration of sliding surfaces and they require regular inspection to maintain the coefficient of friction [401].

\section{Friction pendulum system}

The friction pendulum system (FPS) is a frictional isolation system that combines a sliding action and a restoring force by geometry $[511,512,12]$. In FPS, the isolation is achieved by means of an articulated slider on spherical, concave chrome surface. As the slider moves over the spherical surface, it causes the supported mass to rise and provide a restoring force of the system. The slider is faced with a bearing material, which when becomes in contact with the polished chrome surface, results in a maximum sliding friction coefficient of the order of 0.1 or less at high velocity of sliding and a minimum friction coefficient of the order of 0.05 or less for very low velocities of sliding. The dependency of coefficient of friction on velocity is a characteristic of Teflon-type materials [322]. The natural period of the FPS is fixed [331] and is controlled by selection of the radius of curvature of the concave surface. The enclosing cylinder of the isolator provides a lateral displacement restraint and protects the interior components from environmental contamination. The displacement restraint provided by the cylinder provides a safety measure in case of lateral forces exceeding the design values [263]. 


\section{Resilient-friction base isolation system}

The resilient-friction base isolation base isolation (R-FBI) bearing attempts to overcome the problem of the high friction coefficient of Teflon on stainless steel at high velocities by using many sliding interfaces in a single bearing $[325,329,330]$. Thus the velocity between the top and the bottom of the bearing is divided by a number of layers, so that the velocity at each face is small, maintaining a low friction coefficient [333]. This base isolator consists of concentric layers of Teflon-coated plates that are in friction contact with each other and contains a central core of rubber. It combines the beneficial effect of friction damping with that of resiliency of rubber. The rubber core distributes the sliding displacement and velocity along the height of the R-FBI bearing. They do not carry any vertical loads and are vulcanized to the sliding ring. The system provides isolation through the parallel action of friction, damping and restoring force [263].

\section{Electric-de-France base isolation system}

This system was developed in 1970s under the auspices of "Electric de France" (EDF) [160] and is standardized for nuclear power plants in region of high seismicity. The EDF base isolator essentially uses elastomeric bearing and friction plate in series. It consists of laminated (steel reinforced) neoprene pad topped by lead-bronze plate that is in friction contact with steel plate anchored to the base raft of the structure. The friction surfaces are designed to have a coefficient of friction of 0.2 during the service life of the base isolation system. An attractive feature of EDF isolator is that for lower amplitude ground excitation the lateral flexibility of neoprene pad provides base isolation and at high level of excitation sliding will occur which provides additional protection. This dual isolation technique was intended for small earthquakes where the deformations are concentrated only in the bearings. However, for larger earthquakes the bronze and steel plates are used to slide and dissipate seismic energy.

\section{Sliding resilient-friction base isolation system}

In 1991, [417] proposed the design of the sliding resilient-friction (S-RF) base isolator. This isolator combines the desirable features of the EDF and the RFBI systems. It was suggested to replace the elastomeric bearings of the EDF base isolation by the R-FBI units. It means that the friction plate replaces the upper surface of the R-FBI system in the modified design. As a result, the structure can slide on its foundation in a manner similar to that of EDF base isolation system. For low level of seismic excitation the system behaves as R-FBI system. The sliding at the top friction plate occurs only for a high level 
of ground acceleration that provides additional safety for unexpected severe ground motion.

\section{EERC base isolation system}

This system is a combination of elastomeric and sliding systems. It was developed and tested at the Earthquake Engineering Research Center (EERC). In this system the interior columns of the structure were carried on teflon on stainless steel sliding elements and the exterior columns on the low-damping natural rubber bearings. The elastomeric bearing provides restoring capability while the sliding elements provides energy dissipation [74].

\section{The TASS base isolation system}

The TASS system was developed by the TAISEI Corp. in Japan [244]. This system combines teflon-stainless steel elements to carry vertical load and laminated neoprene bearings which carries no load but is used to provide restoring forces. The pressure on the teflon sliding surface is around $10 \mathrm{MPa}$, and the coefficient of friction ranges from 0.05 at slow sliding speeds to around 0.15 at higher speeds. The main disadvantage of this system is that because the elastomeric bearings carry no vertical load they experience tension and the velocity sensitivity of the sliding surface makes modeling of the system quite difficult.

\section{Variable friction pendulum isolation system}

It is a modified form of the friction pendulum isolation system, in which the shape of the sliding surface is non-spherical to allow for variable vibration period [364]. The variation of the friction coefficient in this system is such that up to a certain value of displacement the frictional force increases and then it decreases with further increase in displacement [349]. This isolation system retains the advantages of both the pure friction isolation system and FPS, due to amplitude dependent time-period and softening mechanism of isolator restoring force. The geometry of the isolator can be chosen to achieve a progressive period shift at different response levels. The advantage of variable frequency of oscillation is particularly significant for sliding surfaces with low coefficients of friction and during high intensity earthquakes.

\section{Sliding concave foundation base isolation system}

It is a foundation system, which includes a lower part and an upper sliding raft. The lower part has a generally concave surface at the top. The upper sliding raft has a convex surface at the bottom that is adapted to rest on the 
concave surface of the lower part and allow for sliding rotational movement therebetween. The building or the object to be isolated is attached to the upper sliding raft [171]. A structure supported on this system behaves like a compound pendulum during seismic excitation. The isolation takes place due to sliding and the energy dissipation is because of friction while the restoring force is attributed to the foundation curvature. However, this system may allow permanent tilting of isolated objects.

\section{Multiple friction pendulum isolation system}

The multiple friction pendulum system (MFPS) was proposed by [428, 429] as an improvement of the FPS. The principal benefit of the MFPS bearing is its capacity to accommodate substantially larger displacements compared to a traditional FPS bearing of identical plan dimensions. The MFPS consists of two concave surfaces and a specially designed articulated slider, which is located between these two concave surfaces. Based on this special design of the articulated slider, pressures on the concave surfaces can be properly distributed during their movements. The MFPS isolator can be designed with unequal radii and unequal friction coefficients at the top and bottom concave surfaces. In this case, the MFPS bearing behaves much like a traditional FPS bearing with effective radius of curvature equal to the sum of the radii of curvature of the two concave surfaces (but modified for the height of the slider) and friction equal to the average of the coefficient of friction at each sliding interface. Additional investigations of the MFPS were carried out by [427, 143, 144, 145].

\section{Uplift-restraining friction pendulum base isolation system}

Based on the Friction Pendulum (FP) principle, the XY-FP isolation bearing was proposed by [377, 376, 378]. This device consists of two orthogonal concave beams interconnected through a sliding mechanism that permits tensile forces to develop in the bearing. Thus preventing potential structure-foundation separation when the bearing is subjected to tension during earthquakes.

\section{The RoGlider isolation system}

The RoGlider is a sliding bearing proposed by [373] and includes an elastic restoring force. The RoGlider either is a double acting unit with the restoring force provided by two rubber membranes or a single acting unit. The double acting RoGlider consists of two stainless steel plates with a PTFE ended puck sitting between the plates. Two rubber membranes are attached to the puck with each being joined to the top or bottom plates. When the top and bottom plates slide sideways with respect to each other, diagonally opposite parts of the membrane undergo tension or compression. The tension components provide 
the restoring force between the plates and the whole system exhibits hysteretic behavior. A typical RoGlider may have a maximum displacement of $\pm 600 \mathrm{~mm}$, a maximum vertical load of $1 \mathrm{MN}$, with an outside diameter around $900 \mathrm{~mm}$ and a coefficient of friction of $0.11 \%$ at $0.5 \mathrm{~ms}^{-1}$.

\subsubsection{Spring-based isolation systems}

These types of isolation systems are generally used when full three-dimensional isolation is required, as the case of large generators in power plants. These systems uses large helical steel springs that are flexible in both horizontal and vertical directions. The steel springs are completely without damping and they are used in conjunction with other damping devices. As in all three-dimensional systems, there is a very strong coupling between horizontal motion and rocking motion. Therefore, the spring isolation systems are not effective in reducing the accelerations in buildings due to the rocking motion [310].

\subsubsection{Rolling-based isolation systems}

The concept of rolling is an efficient way to achieve the necessary structurefoundation decoupling if compared to the sliding motion mechanism, as the force required to initiate rolling motion is significantly smaller than that required to start sliding. This is mainly attributed to the very small rolling friction if compared to the sliding one. In general, rolling friction is far less than sliding friction and the former is only about 1/40-1/60 of the later [472].

\section{Rolling rods isolation systems}

In 1993, [291] has proposed a new system of free circular rolling rods located between the base and the foundation. The most attractive feature of this type of isolator is their low value of rolling friction coefficient, which allows a very low earthquake force to be transmitted to the superstructure. However, such a system lacks re-centering and damping capabilities, resulting in residual and large peak displacements. To provide them with re-centering mechanism, [225] proposed that the shape of rolling rods should be elliptical rather then circular. The low value of the rolling friction coefficient ensures the transmission of a limited earthquake force into the superstructure and the eccentricity of the elliptical rolling rods provides a restoring force that reduces peak base displacements and brings the structure back to its original position. However, the system damping is still absent. 


\section{Rolling pendulum isolation systems}

Combining the advantage of free rolling rod isolators and FPS isolators, the base isolation by a ball system with restoring property was proposed by [517]. The resulting system is a self-recentering ball bearing having a fixed vibration period, as the FPS, which represents a severe practical difficulty. This bearing consists of a spherical rolling body between two opposite spherical grooves. A similar approach was presented by [30] where a mushroom-shaped (pendulumlike) basements were used under structures as a base isolation system. Each basement have a spherical arc that rests on the foundations in a tangential manner. From the arc midpoint there is a radial pinned arms that is connected to the structure column. The whole system acts like a spring under the structure. The main problems in using of this method are the lack of damping and stress concentration at the contact point between spherical base and foundation. Indeed, the idea of the rolling pendulum system is quite old, as it dates back to the US patent [426].

\section{Ball-N-Cone isolation systems}

A seismic isolation bearing consisting a steel ball sandwiched between two horizontal, steel load plates. The load plates have a shallow, concave, conical recess of vertical, collinear axis. The cone axis passes through the ball centerline at rest. The upper and lower load plates are bolted to the structure and base, respectively. Gravity restores the bearing's displacement in the lack of external forces. Lateral bearing forces are independent of displacements [254, 238, 237].

\section{Multi-step isolation systems}

A manufacture to protect a structure from destructive earthquakes as well as to secure its stability under the strongest possible wind, having a number of ball transfer units rigidly coupled to a supported superstructure; the same number of pedestal plates supporting the ball transfer units, each having an upper surface having a plurality of curved surfaces with a central depression following the shape of the ball. The force of gravity will keep the structure in its steady initial position when the balls are inset into corresponding depressions at any wind pressure and at slight earthquakes. With magnitude of earth movement exceeding a certain threshold the balls get out of central depressions thus providing a controlled separation of the superstructure from the foundation, and the upper surfaces of pedestal plates with properly designed geometry governs transfer of horizontal movement into the superstructure and prevents hazardous structural vibrations [390]. 


\section{Mutually eccentric rotators isolation systems}

A base isolator that has four base isolation units placed at four corners between a floor slab and a floor plate. Each base isolation unit has a middle plate, a lower support body which is rotatable in a first direction on the lower side of the middle plate, and an upper support body rotatable in a second direction on the upper side of the middle plate. The upper support body and the lower support body each have mutually eccentric rollers. The middle plates of the four base isolation units are separated from one another so that the plates can be independently displaced or moved [240].

\section{Directional rolling pendulum isolation systems}

They are bi-directional rolling pendulum seismic isolation systems for reducing seismic force acting on a structure by rolling pendulum movements. The system has a lower plate forming a rolling path in a first direction; an upper plate forming a rolling path in a second direction; and a roller assembly performing a pendulum motion by rolling and moving along the lower and upper plates wherein the roller assembly performs the pendulum motion when seismic load is applied, thereby reducing the seismic load of a structure [259].

\section{RoBall isolation systems}

It consists of two opposite saucer-like rubber containers with flat outer surfaces and curved inner ones [372]. It contains seven solid balls. Other designs of the RoBall suitable for larger displacements could include 13, 19, 25 or more solid balls in close packed arrays. The sides of the RoBall may be made thicker than the top and bottom surfaces, thereby contributing to a restoring force for small displacements, while for large displacements there is cyclic restoring with a force-displacement wavelength approximately twice the diameter of the RoBall. The rolling action of the RoBall means that the device itself has no displacement limit and so the maximum displacement is limited only by installation requirements. The dynamic behavior of the device is independent of both frequency and ambient temperature within ranges that are applicable to most practical installations. The effective friction coefficient is around 0.10.

\section{Other rolling-based isolation systems}

Ref. [110] proposed an isolator to prevent transmitted vibrations that includes a ring which fits within a shaft opening of a housing. A bearing is received within the isolator and a shaft is received within the bearing. The vibrations are decoupled from a vibration transmission path by the different material of 
the isolator. The isolator is preferably manufactured of a rigid laminate material dissimilar to the housing material. Another embodiment of the present invention integrates the isolator directly into the bearing assembly. By manufacturing the outer member of the bearing assembly of the laminated material, the outer member similarly breaks the transmission path of vibrations without the need of the additional member. Noise is thereby substantially prevented from reaching, and from being amplified by, the housing. This greatly reduces the resulting noise and vibration.

A seismic isolation bearing comprises a lower plate, an upper plate, and a cylindrical roller in rolling contact with an upwardly facing, bearing surface of the lower plate and a downwardly facing surface of the upper plate was proposed by [273]. The lower plate is fixable to a base, while the upper plate is fixable to a superstructure. One or both bearing surfaces are sloped to form a central trough at which the cylindrical roller resides under normal weight of the superstructure, and toward which the roller is biased when displacement between the plates occurs. A pair of sidewall members are fixed to the lower plate to withstand strong forces directed laterally with respect to the isolation axis along which rolling displacement occurs, and a pair of sliding guides carried one at each end of the roller provide dry frictional damping as they engage an inner wall surface of a corresponding sidewall member. A similar isolation bearing was presented in [490].

A rolling-based seismic isolation apparatus is structured by [348] such that a pair of seismic isolation mechanisms are formed each having a pair of upper and lower clamping members formed in the shape of a rectangular frame, wherein upper and lower roller guide surfaces, in each of which a longitudinal central portion is formed as a concavely arcuate curved surface and portions respectively extending therefrom are formed as convexly arcuate curved surfaces, are formed on the four sides of the upper and lower clamping members, and wherein rollers each having a circular cross section are respectively interposed between the upper and lower roller guide surfaces. The pair of seismic isolation mechanisms are installed by being superposed on top of each other such that the rolling directions of the rollers in the two seismic isolation mechanisms are perpendicular to each other. During the occurrence of an earthquake, the rollers are adapted to roll in contact with the upper and lower roller guide surfaces so as to absorb and dampen seismic waves in the longitudinal and transverse directions of the apparatus. Two similar isolation systems were proposed by $[169,345]$.

Ref. [493] proposed a vibration isolation unit in which a rolling element having a first curved surface that is a convex surface is interposed between a first and second members, and the rolling element is allowed rolling with respect to the first member in the first curved surface thereof. The rolling element has a second curved surface that faces the first curved surface and is formed of a curved surface center different from that of the first curved 
surface. As the rolling element, resulting from an additional force applied on the first and second curved surfaces, rolls with respect to the first member, the second member vibrates with a restoring force determined based on an average curvature radius of the first and second curved surfaces of the rolling element and a distance between curved surface centers of the first and second curved surfaces.

\subsubsection{Synthetic liners and artificial soil layers}

Placing smooth synthetic materials beneath the foundation of structures was proposed by [495] to provide a friction base-isolating layer. It was concluded that a high strength, non-woven geo-textile placed over an ultra molecular weight polyethylene was most suitable and gave a static and dynamic coefficient of friction of 0.10 and 0.07 , respectively. This coefficient was almost independent of sliding velocity and normal stress. This system has the advantage in that the sliding surface is placed below a concrete slab and the system is therefore less likely to slide excessively in wind storms. Similar systems were proposed by $[257,494,496]$.

Interposing an artificial soil layer between the superstructure and the foundation of the buildings was proposed by [128]. This soil layer has a low shearing resistance, which allows the building to slip under the action of strong seismic motions. However, the system constructability is dubious and the design is problematic. Moreover, the coefficient of friction of the system is 0.2 , which does not provide large force reductions. A similar base-isolation layer but only for heavy construction was suggested by [467]. [430] proposed a similar seismic isolation method particularly suitable for developing countries. This method makes use of rubber-soil mixtures as a thick layer beneath the structure to provide horizontal and vertical seismic isolation effect.

\subsubsection{Rocking isolation system}

Rocking mechanism can be an effective means of seismic isolation, despite the fact that it is rarely conceived a possible alternative [87]. Tall slender structures suffer tension at the foundation level under lateral loads. It is extremely expensive to provide tension capacity in building foundation for such purpose. As an alternative, it is possible to allow the building columns or bridge piers to step off the foundation [333]. This form of partial isolation reduces the seismic loads throughout the structure. The dynamics of the stepping structures have been extensively studied theoretically [500] and experimentally [252, 103]. 


\subsubsection{Sleeved-pile isolation system}

In situations where it is necessary to use deep pile foundations for buildings on very soft soil, it can be advantageous to use these piles to provide the horizontal flexibility needed for the isolation system. The piles are made flexible by enclosing them in tubes with suitable gap for clearance [46, 77].

\subsubsection{Other isolation systems}

In 2004, [10] proposed an isolation system that uses a commercially available air-spring of diameter $600 \mathrm{~mm}$ to act as a belt on a fabric bag filled with a chosen percentage of liquid and air. Although it was claimed that the results were satisfactory, this system is not robust and is unlikely to be acceptable.

Rocking pillars were used as an isolation system to support a superstructure by [241]. The pillar is from a steel tube filled with concrete with spherical caps. A damper between the pillars and caisson is required to limit movement and provide restraint to wind movement. The system motion is rolling-based and is self-recentering. The natural period with dampers was rather low for a base-isolated structure $(0.63 \mathrm{sec})$ and indicated limited isolation. Although this system has potential as bridge piers, it is expected that this type of construction be expensive in practice and to be beset by practical problems. Waterproofing the system would also be a problem. A similar isolation system was proposed by [359] as a low-cost solution for seismic protection of low-income people housing.

Based on employing the first soft story in a building as an isolation system, [321] proposed a system for the design of earthquake-resistant buildings. In this system, first story shear walls are fitted with flat Teflon sliders while the remaining first story columns are designed with reduced yielding stress. In this system a major part of the weight of the building is carried by the Teflon sliders, which makes the system behave almost like a plane friction isolation system, but with one more advantage that is the remaining first story columns may provide a restoring mechanism during small earthquakes. However, the partial structure-foundation separation provided by this system makes it only valid for low-intensity earthquakes.

A base-isolation device that takes advantage of the reduced acceleration experienced by masses moving on inclined planes and which systematically transforms kinetic energy into potential energy has been proposed by [304]. In its simplest form, the device consists of two wedges sliding on a horizontal plane in opposite directions and constrained from retreating by ratchets or bilinear dampers. The superstructure rests at the intersection of the two wedges. For a sufficiently large horizontal acceleration of the base, the structure starts to move up the inclined plane of one of the wedges, which remains fixed while the second wedge is slaved to follow the structure. As the direction of the base acceleration reverses, the process is reversed and the structure starts to climb 
on the second inclined plane while the first wedge follows. The overall result is that the horizontal acceleration of the structure is reduced with respect to that of the base and that kinetic energy associated with horizontal velocities is systematically transformed into potential energy.

A base isolation device was proposed, deeply examined and modeled by [71, $69,72]$. It consists of two disks, one vertical cylinder with an upper enlargement sustained by three horizontal cantilevers, and at least three inclined shape memory alloy (SMA) bars. The role of the SMA bars is to limit the relative motion between the base and the superstructure, to dissipate energy by their super-elastic constitutive law and to guarantee the re-centring of the device. A prototype was built and tested under sinusoidal waves of displacement of increasing frequency with different amplitudes. Further, application of the device to a highway bridge benchmark problem was presented by [70]. 
This page intentionally left blank. 


\section{A new device for seismic isolation}

\subsection{Motivations for a new isolator}

Nowadays, many new materials and devices continue to be proposed for use in base isolation. Based on the extent of control to be achieved over the seismic response, the choice of the isolation system varies and thereupon its design is done to suit the requirements of use of the structure. A practical seismic isolation system should meet the following four requirements:

1. Sufficient horizontal flexibility to increase the structural period.

2. Sufficient energy dissipation capacity to limit the isolator displacements to a practical level.

3. Adequate rigidity under general service loading.

4. Adequate vertical stiffness to support the isolated object weight.

Most commonly used seismic isolating systems can satisfy all the above requirements while having their own characteristics. However, no one type device is perfect as each device has its own drawbacks. Therefore, there is always a continuous need to enhance the existing isolation systems or even to innovate others to get the most benefits of seismic isolation while avoiding any unwanted negative effects. Table 3.1 summarizes the advantages and disadvantages of the most commonly used device types. These advantages and disadvantages are brief, general and may not be comprehensive. Further, the listed disadvantages may apply to a generic type, some manufactures may have specific procedures to alleviate the disadvantages. 
Table 3.1: Advantages and disadvantages of the most commonly used isolation systems

\begin{tabular}{|c|c|c|}
\hline Device & Advantages & Disadvantages \\
\hline Elastomeric & $\begin{array}{l}\text { - Low structural accelerations } \\
\text { - Relatively low cost }\end{array}$ & $\begin{array}{l}\text { - Large displacements } \\
\text { - Low damping } \\
\text { - No recentering mechanism } \\
\text { - Shear strain reduces capacity } \\
\text { - Minimum flexibility limit } \\
\text { - No resistance to service loads } \\
\text { - No buffer } \\
\text { - P- } \Delta \text { influence }\end{array}$ \\
\hline HDR & $\begin{array}{l}\text { - Moderate structural accel. } \\
\text { - Resistance to service loads } \\
\text { - Moderate to high damping }\end{array}$ & $\begin{array}{l}\text { - Strain dependent stiffness } \\
\text { - Strain dependent damping } \\
\text { - Complicated analysis } \\
\text { - Scragging-change properties } \\
\text { - Narrow range of stiffness } \\
\text { - Narrow range of damping } \\
\text { - No buffer } \\
\text { - P- } \Delta \text { influence }\end{array}$ \\
\hline LRB & $\begin{array}{l}\text { - Moderate structural accel. } \\
\text { - Resistance to service loads } \\
\text { - Wide range of stiffness } \\
\text { - Wide range of damping } \\
\text { - High damping levels }\end{array}$ & $\begin{array}{l}\text { - Cyclic change in properties } \\
\text { - Bearing area reduction } \\
\text { - P- } \Delta \text { influence } \\
\text { - Not for low-mass structures } \\
\text { - No buffer }\end{array}$ \\
\hline $\begin{array}{l}\text { Flat } \\
\text { Sliders }\end{array}$ & $\begin{array}{l}\text { - Simple in concept } \\
\text { - Resistance to service loads } \\
\text { - No strain hardening } \\
\text { - Low profile } \\
\text { - High damping levels } \\
\text { - Earthquake independent } \\
\text { - Structure independent }\end{array}$ & $\begin{array}{l}\text { - High structural accel. } \\
\text { - Changing friction coefficient } \\
\text { - High initial stiffness } \\
\text { - No recentering mechanism } \\
\text { - No buffer }\end{array}$ \\
\hline $\begin{array}{l}\text { Curved } \\
\text { Sliders }\end{array}$ & $\begin{array}{l}\text { - Low profile } \\
\text { - Resistance to service loads } \\
\text { - Relatively wide damping range } \\
\text { - Reduced structural torsion } \\
\text { - High damping levels }\end{array}$ & $\begin{array}{l}\text { - High structural accel. } \\
\text { - Changing friction coefficient } \\
\text { - High initial stiffness } \\
\text { - High cost } \\
\text { - Fixed vibration period } \\
\text { - Uplifted structure with motion } \\
\text { - Likely permanent eccentricity }\end{array}$ \\
\hline Rollers & $\begin{array}{l}\text { - Very low structural accel. } \\
\text { - Simple means and concept } \\
\text { - Great horizontal flexibility }\end{array}$ & $\begin{array}{l}\text { - No damping } \\
\text { - No buffer } \\
\text { - No recentering mechanism } \\
\text { - Not for heavy masses }\end{array}$ \\
\hline
\end{tabular}




\begin{tabular}{lll}
\hline Device & Advantages & Disadvantages \\
\hline \multirow{3}{*}{ Springs } & & - Flattening of contact surfaces \\
& - Provide 3D isolation & - No damping \\
& & - Produces vertical accelerations \\
& & - No buffer \\
Hysteretic & - Control displacements & - No recentering mechanism \\
Dampers & - Low cost & - Add force to system \\
& - Provide stiffness and damping & \\
& - Wide damping range & \\
& - Widely available & \\
\hline
\end{tabular}

Table 3.1: (continued)

Rough inspection of Table 3.1 confirms the fact that each of the seismic isolation systems mentioned above has specific dynamic properties and functions but no device is perfect. This motivates the efforts either to enhance the existing devices or to innovate others with the aim of attaining the maximum protection level of structures through seismic isolation. Unfortunately, most of the isolation systems reported in the literature are patented products (the same is also true with most newly invented products), not all of them are readily available for procurement and direct enhancement. Therefore, the intention may be directed toward creating more efficient isolation devices. This chapter presents an attempt to innovate a practical isolation device that aims to get the best of the present day isolation systems while avoiding their main drawbacks.

\subsection{Concepts of the proposed isolator}

The proposed isolation bearing is referred to as roll-n-cage (RNC) isolator. It is recently patented through the Spanish Office of Patents and Marks [211]. The starting point of designing such isolation system was to seek a motion mechanism that provides the maximum structure-foundation decoupling. The concept of rolling is an efficient way to achieve the necessary structure-foundation decoupling if compared to the sliding motion mechanism, as the force required to initiate rolling motion is significantly smaller than that required to start sliding. This is mainly attributed to the very small rolling friction if compared to the sliding one. In general, rolling friction is far less than sliding friction 
and the former is only about $1 / 40-1 / 60$ of the later [472]. Therefore, the proposed RNC isolator is a rolling-based isolation bearing. The most attractive feature of this isolator is its ability to allow a very low earthquake force to be transmitted to the superstructure.

Briefly, owing to its distinct configuration, the RNC isolator possesses unique properties for a seismic isolator, including:

1. Multi- and unidirectional isolation.

2. Energy dissipation.

3. Uplift restraint.

4. Resistance to wind and minor vibrations.

5. Built-in buffer.

6. Inherent gravity-based recentering mechanism.

7. Suitable for light, moderate and heavy mass systems.

8. Resistance to flattening of contact surfaces.

9. Wide range of stiffness and damping.

10. Independent damping and bearing mechanisms.

11. Independent stiffness and bearing mechanisms.

12. Great system-base decoupling.

13. Non-fixed vibration period.

14. Expected reasonable construction cost.

\subsection{Technical field}

The proposed RNC bearing is intended to mitigate or reduce the damage potential and casualties of structural and/or nonstructural systems due to dynamic shock loading, especially seismic loading. The structural systems can be buildings, bridges, water tanks . . . etc, such that the systems to be protected are valid for seismic isolation. The nonstructural systems can be any motion-sensitive equipment, hardware and/or antiquities.

\subsection{Main forms}

The proposed RNC isolator has two main forms, having the same core and spirit, that are distinguished according to the needed vertical load capacity. The first of these two forms is referred to as $\mathrm{RNC}-\mathrm{c}$ and is devoted to support light to moderate mass systems. The RNC-c is shown schematically in Fig. 3.1 , where the vertical loads are only supported by the rolling body of the $\mathrm{RNC}$ isolator. The second form of the RNC isolator is designed for heavy mass systems and is referred to as RNC-a and RNC-b as shown in Figs. 3.2 and 
3.3 , respectively. In these bearings ( $\mathrm{RNC}-\mathrm{a}, \mathrm{RNC}-\mathrm{b})$, the vertical load capacity is further improved by means of a hollow elastomeric cylinder, of designed thickness, around the rolling body of the RNC isolator to represent the main bearing mechanism, where the rolling body itself works as a secondary bearing mechanism. Moreover, the RNC isolator can be designed to provide both multidirectional horizontal isolation, as shown in Figs. 3.1-3.3, and unidirectional horizontal isolation, as illustrated by Fig. 3.4.

\subsection{Main components}

Detailed and expanded plots of the different RNC isolator forms are shown in Figs. 3.5-3.7. From these figures, the RNC isolator comprises the following components:

1. An upper rigid load plate (2) is secured to the superstructure, or to the object to be protected, through the upward facing rigid surface (2-5) and by means of the anchors (10).

2. A lower rigid load plate (3) is secured to the foundation, on which the superstructure or the object to be protected is supported, through the downward facing rigid surface (3-5) and by means of the anchors (10).

3. A quasi-elliptical stiff rolling body (1) is sandwiched between the two bearing plates $(2,3)$. The rolling body has two upper and lower rolling surfaces that face the downward surface of plate (2) and the upward surface of plate (3), respectively.

4. An upper plate (4) of less stiff material (a strong synthetic rubber material such as neoprene) of uniform thickness is inserted between the rolling body (1) and the upper stiff plate (2). The upper surface of (4) is perfectly stuck to the lower surface of plate (2) and is exactly shaped like that surface, which it shares with plate (2). The lower surface of (4) is kept in rolling contact with the upper surface of the rolling body (1).

5. A lower plate (5) of less stiff material (a strong synthetic rubber material such as neoprene), of uniform thickness equal to that of plate (4), is inserted between the rolling body (1) and the lower stiff plate (3). The lower surface of (5) is perfectly stuck to the upper surface of plate (3) and is exactly shaped like that surface, which it shares with plate (3). The upper surface of (5) is kept in rolling contact with the lower surface of the rolling body (1).

6. A hollow elastomeric cylinder (9), of designed in-plan thickness and height, that surrounds the rolling body (1), in the cases of RNC-a and RNC-b 
as shown in Figs. 3.6 and 3.7 respectively. It is made of reinforced elastomeric rubber while its upper and lower ends are perfectly attached to the upper and lower stiff plates $(2,3)$ through suitable grooves as shown in Figs. 3.6 and 3.7.

7. Metallic yield dampers (6a) in the cases of $\mathrm{RNC}-\mathrm{c}$ and $\mathrm{RNC}-\mathrm{a}$ shown in Figs. 3.5 and 3.6, respectively. They are arranged along the perimeter of the isolation bearing and are attached to the rigid bearing plates $(2,3)$ through connection (11) as demonstrated by Fig. 3.8, or via any equivalent rigid connection. The number and cross section of these yield dampers are designed based on the required resistance to minor shaking and the desired protection level. The dampers (6a) are shaped to allow for enough extension during rolling and to reduce the stress concentration at different bends. Accordingly, the used yield dampers (6a) are provided with outside triple curvature (6-1).

8. Lead bar dampers (6b), in the case of RNC-b as shown by Fig. 3.7, that penetrate the full height of the hollow elastomeric cylinder defined in Item 6 and replace the metallic yield dampers defined in Item 7 . The ends of the lead bars fit into suitable grooves in the upper and lower plates $(2,3)$ in Fig. 3.7.

9. The lower surface of the upper stiff plate (2) and the upper surface of the lower stiff plate (3) are provided with properly designed slopes to form an edge troughs, that exactly absorb the expected elevation of the supported object during rolling of the quasi-elliptical rolling body (1), as shown in Fig. 3.9.

10. The lower stiff plate (2) and the upper stiff plate (3) are provided with vertical side walls to help forming a maximum limit of rolling displacement as demonstrated by Fig. 3.10.

11. The stiff rolling body is provided with two right-angle grooves. Such grooves form a lock with the side walls, defined in Item 10, upon reaching the predetermined lateral displacement limit of the rolling body (1) as demonstrated by Fig. 3.10.

12. The upper and lower stiff plates $(2,3)$, in the cases of $\mathrm{RNC}-\mathrm{c}$ and $\mathrm{RNC}-\mathrm{a}$ in Figs. 3.5 and 3.6, are provided with edge stiffeners $(7,8)$, respectively. These stiffeners improve the rigidity of the outer edges of plates $(2,3)$, with less construction material, where the metallic yield dampers (Item 7) are connected.

13. The upper and lower spherical surfaces (1-3) of the stiff rolling body (1), that are in rolling contact with the surrounding less stiff plates $(4,5)$, are coarse regular surfaces to improve the sliding friction coefficient. 
14. The plane coarse surfaces (1-2) surround the coarse regular spherical surfaces (1-3) of the rolling body (1), in order to satisfy the required bearing area for vertical loads at the positions of maximum lateral displacement, Figs. 3.9(a,c).

15. The edge troughs of the upper plates $(2,4)$ and lower plates $(3,5)$ are provided with plane horizontal parts $(2-3,4-3)$ and $(3-3,5-3)$ respectively. These plane surfaces correspond to what defined in Item 14.

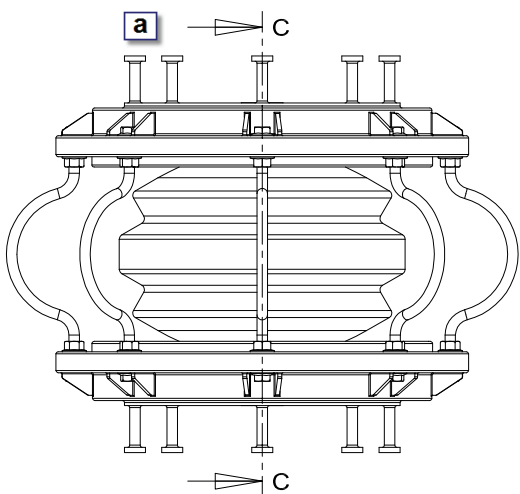

Front View, RNC Type (c)

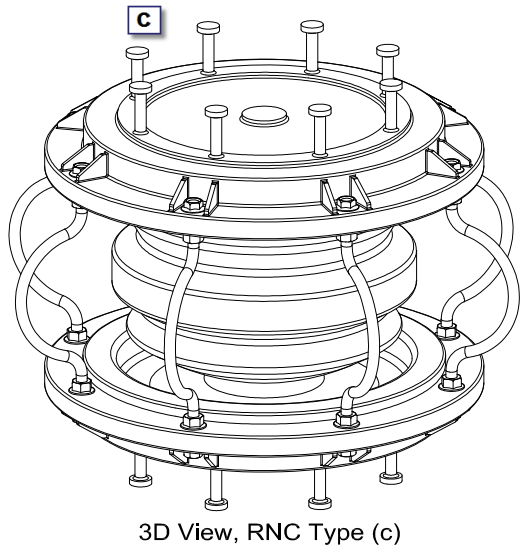

3D View, RNC Type (c)

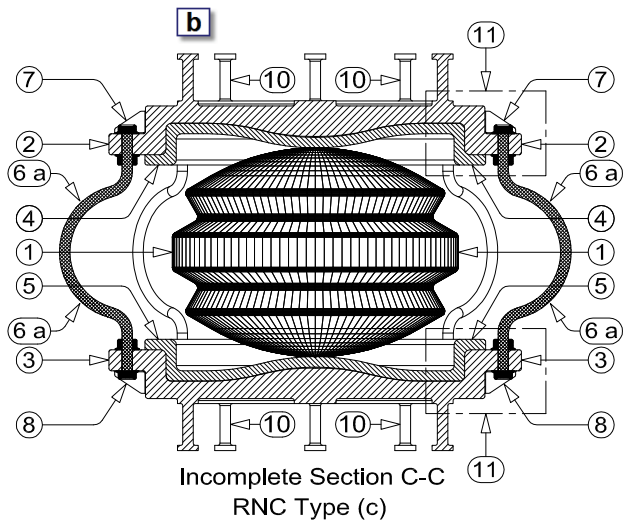

d

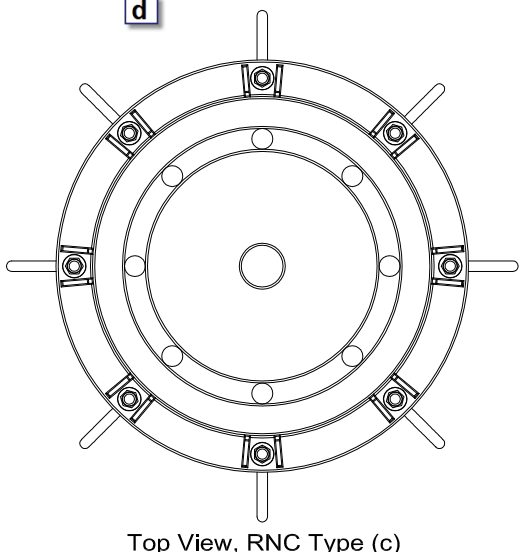

Top View, RNC Type (c)

Figure 3.1: RNC isolator for light to moderate mass systems type c, RNC-c

\subsection{Principles of operation}

From its name, the roll-n-cage isolation bearing adopts the rolling mechanism to cut off the load path between the superstructure and the base. Such rolling 

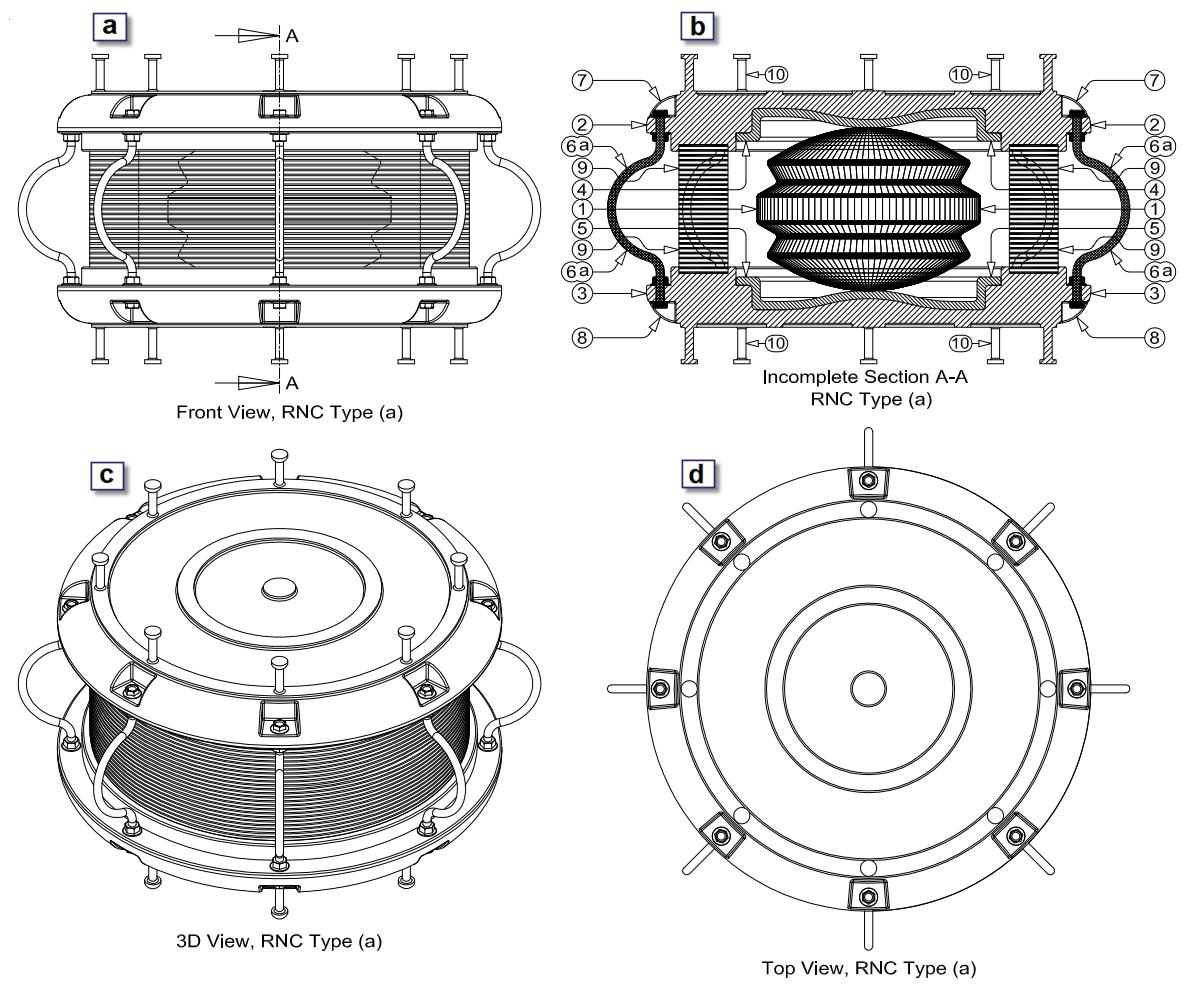

Figure 3.2: RNC isolator for heavy mass systems type a, RNC-a

mechanism offers minimal degree of superstructure-base coupling, as it requires lower force to roll if compared to sliding mechanism, which translates into much reflection of seismic forces. As a consequence, rolling approaches more the ideal isolation concept which requires total horizontal separation. However, a system with minimum resistance to lateral motion is susceptible to shaking under minor vibrations and may end up in a different location after an earthquake and continue to dislocate under aftershocks. To avoid these side effects in the RNC isolator, it is provided with a number of metallic yield dampers (6a) or lead bars (6b), as a cage around the rolling body, to provide suitable elastic stiffness under minor vibrations, in addition to damping to limit the vibrational displacement amplitude. They are shaped and arranged as shown in Figs. 3.1, $3.2,3.4$ to exhibit the same shear strain in any horizontal direction.

The RNC isolator has a buffer mechanism to limit the displacement to a predetermined value under severe earthquakes, as illustrated in Figs. 3.9(a,c), 3.10 , beyond this limit the buffer mechanism stops the isolated structure, or object, with minimal shock.

Practically, and after being dislocated, the isolated superstructure must 

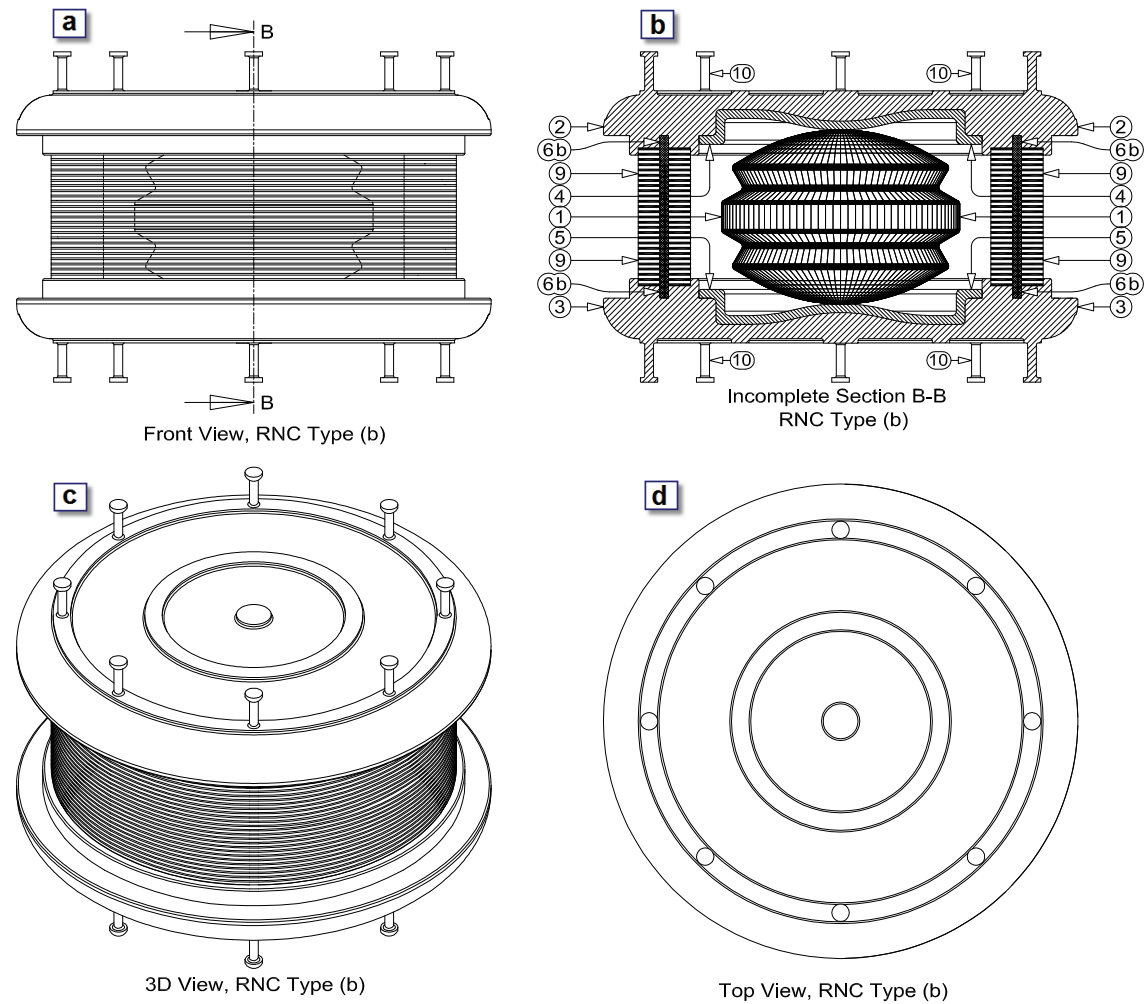

Figure 3.3: RNC isolator for heavy mass systems type b, RNC-b

return back to its original position before excitation. So, the RNC is provided with an efficient gravity-based recentering mechanism through the elliptically shaped rolling body (1), along with the weight of the supported superstructure (or object) to generate a restoring couple $M_{\mathrm{r}}$ opposite to the motion-causing couple as seen in Fig. 3.11.

During the maximum displacement positions, the less stiff plates $(4,5)$ act as shock absorbers as illustrated in Figs. 3.9(a,c), 3.10. A strong synthetic (hyperelastic) rubber material, such as neoprene, is recommended for these plates $(4,5)$. These plates $(4,5)$ increase the sliding friction coefficient with the rolling body (1), which also has regular coarse rolling surfaces in order to force the rolling motion and to avoid any unwanted slip of the rolling body (1). In addition, plates $(4,5)$ eliminate the stiff-to-stiff pointwise contact between the stiff body (1) and the stiff plates $(2,3)$, and therefore prevent flattening at such contact points.

As the elliptical rolling body rolls back and forth, the isolated superstructure is elevated upward. This negative effect is alleviated in the RNC isolator, 
a

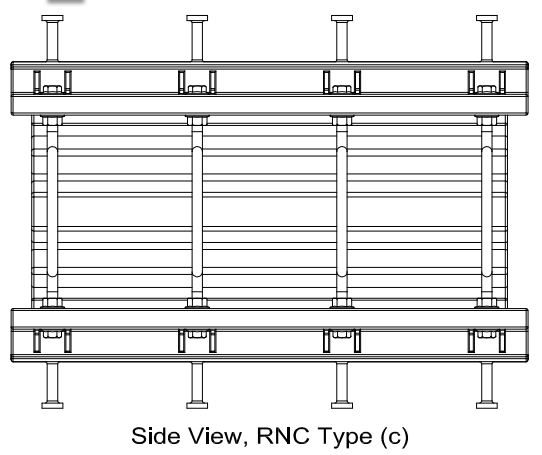

C

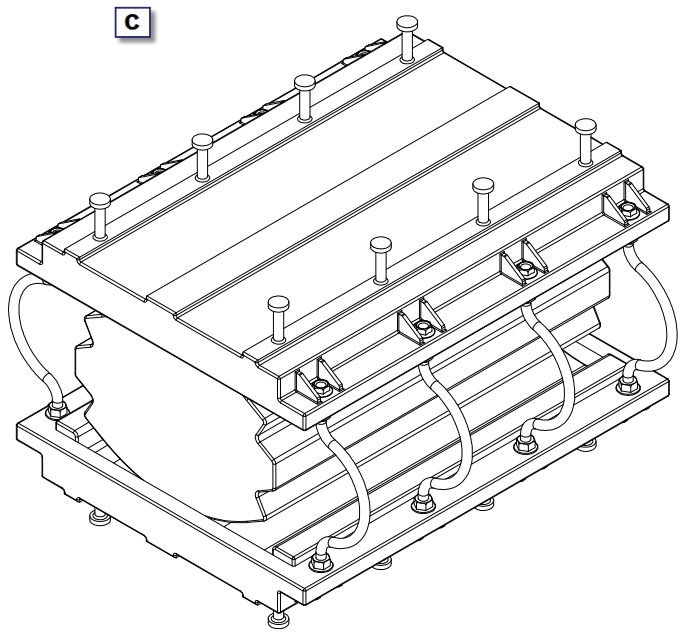

3D View, RNC Type (c)

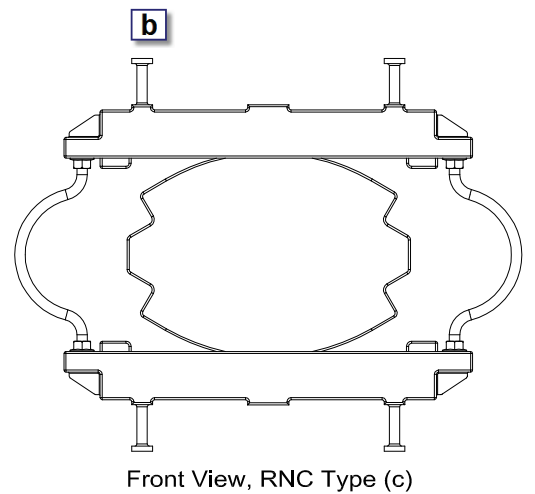

d

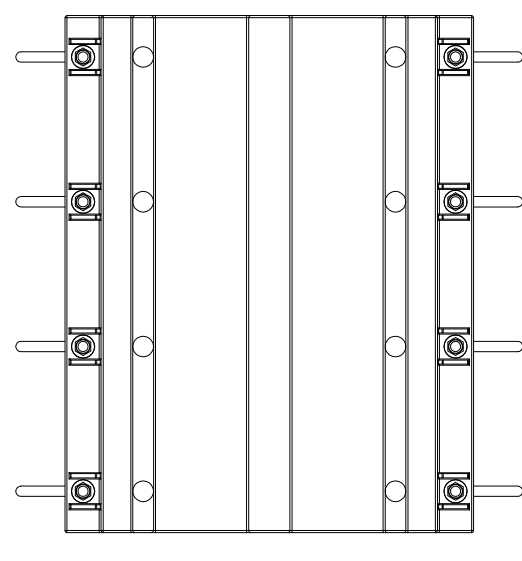

Top View, RNC Type (c)

Figure 3.4: Unidirectional RNC isolator, type c

along the full motion time history, by the properly designed curvatures of the inner faces of the upper and lower stiff plates $(2,3)$, see Fig. $3.5-3.7$ and 3.9(a,b,c). These curvatures prevent the pendulum motion (which results a fixed vibration period) of the RNC-isolated structure. Therefore, the RNC isolator does not have a fixed vibration period. Moreover, such geometry absorbs exactly the vertical elevation of the isolated superstructure (or object), due to rolling of the elliptical body (1), keeping the same vertical offset between the upper and lower plates $(2,3)$ as illustrated in Fig. 3.9(c,d,e) by dashed horizontal lines. This guarantees that the RNC isolator does not modify the vertical component of the acceleration as recommended by international codes.

Since the RNC isolator is rolling-based bearing, the rocking motion of the 

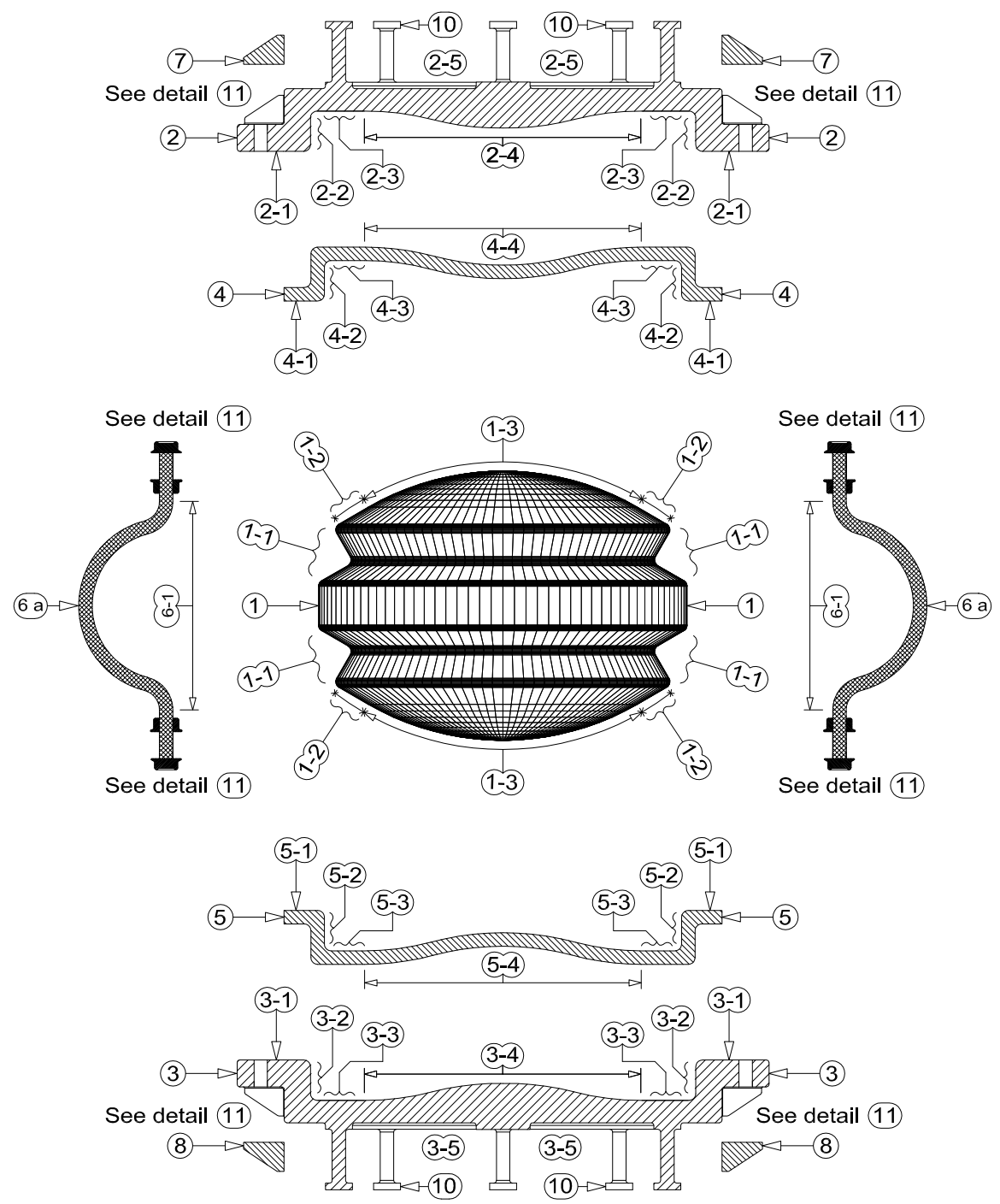

Figure 3.5: Details of the RNC type c, RNC-c

isolated object is not likely to occur during earthquakes. However, if such motion is anticipated, the RNC isolator is capable of supporting reasonable tensile forces, which develop due to rocking of the isolated structure, by means of metallic yield dampers.

A stiff rolling body sandwiched between two horizontal stiff plates has a point contact with each of them, which is not sufficient to support higher 

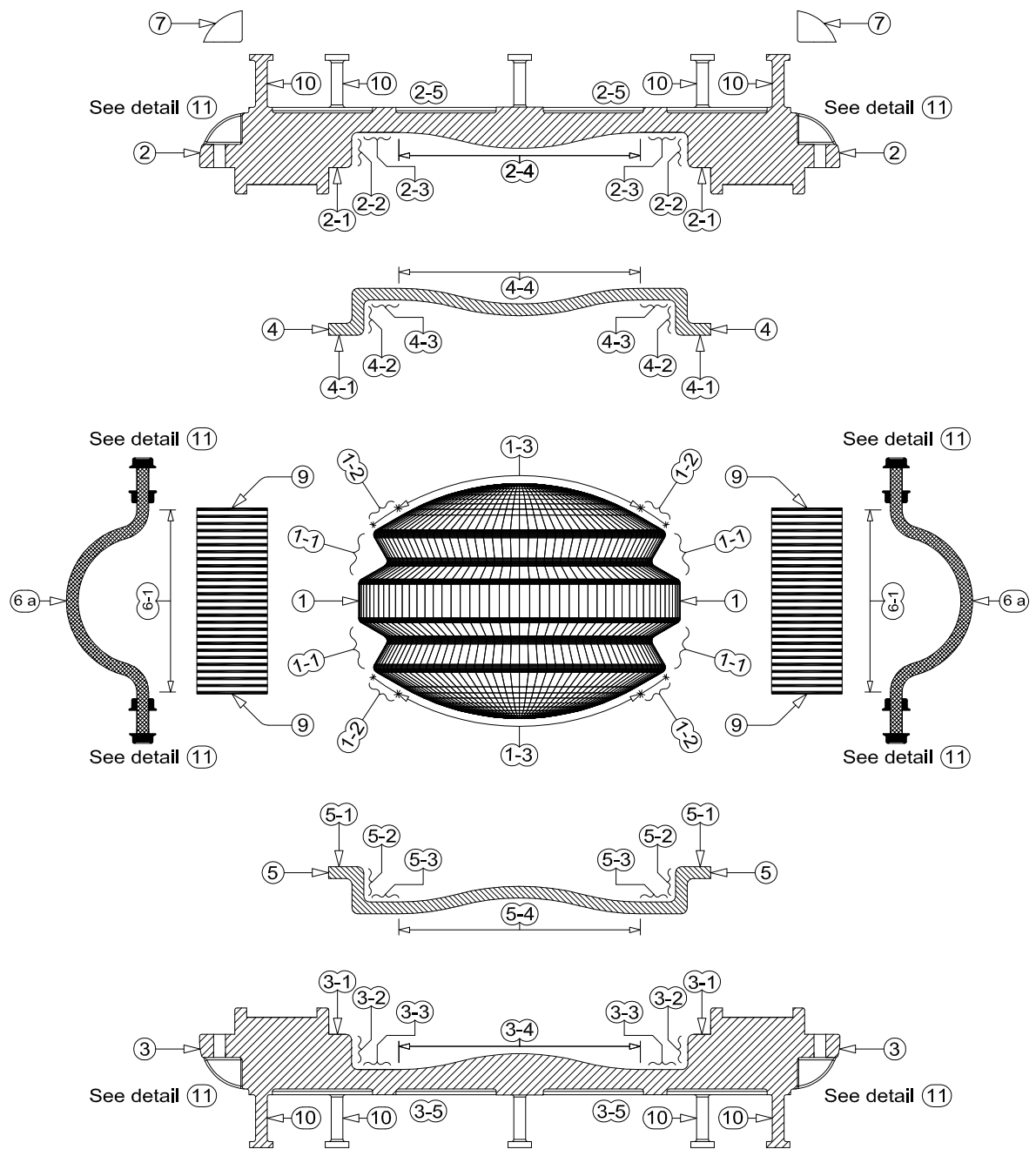

(8) $D$ - 8

Figure 3.6: Details of the RNC type a, RNC-a

vertical loads. In the RNC isolator, the bearing mechanism is enhanced by inserting the less stiff (deformable) plates $(4,5)$ between the rolling body $(1)$ and the upper and lower stiff plates $(2,3)$, respectively. This represents the main bearing mechanism in the case of $\mathrm{RNC}-\mathrm{c}$ (Figs. 3.1, 3.5), while it represents a secondary bearing mechanism in the cases of RNC-a and RNC-b. The proportion of vertical load that is taken by this bearing mechanism is the unique source of the restoring moment $M_{\mathrm{r}}$, along with the eccentricity provided by the 

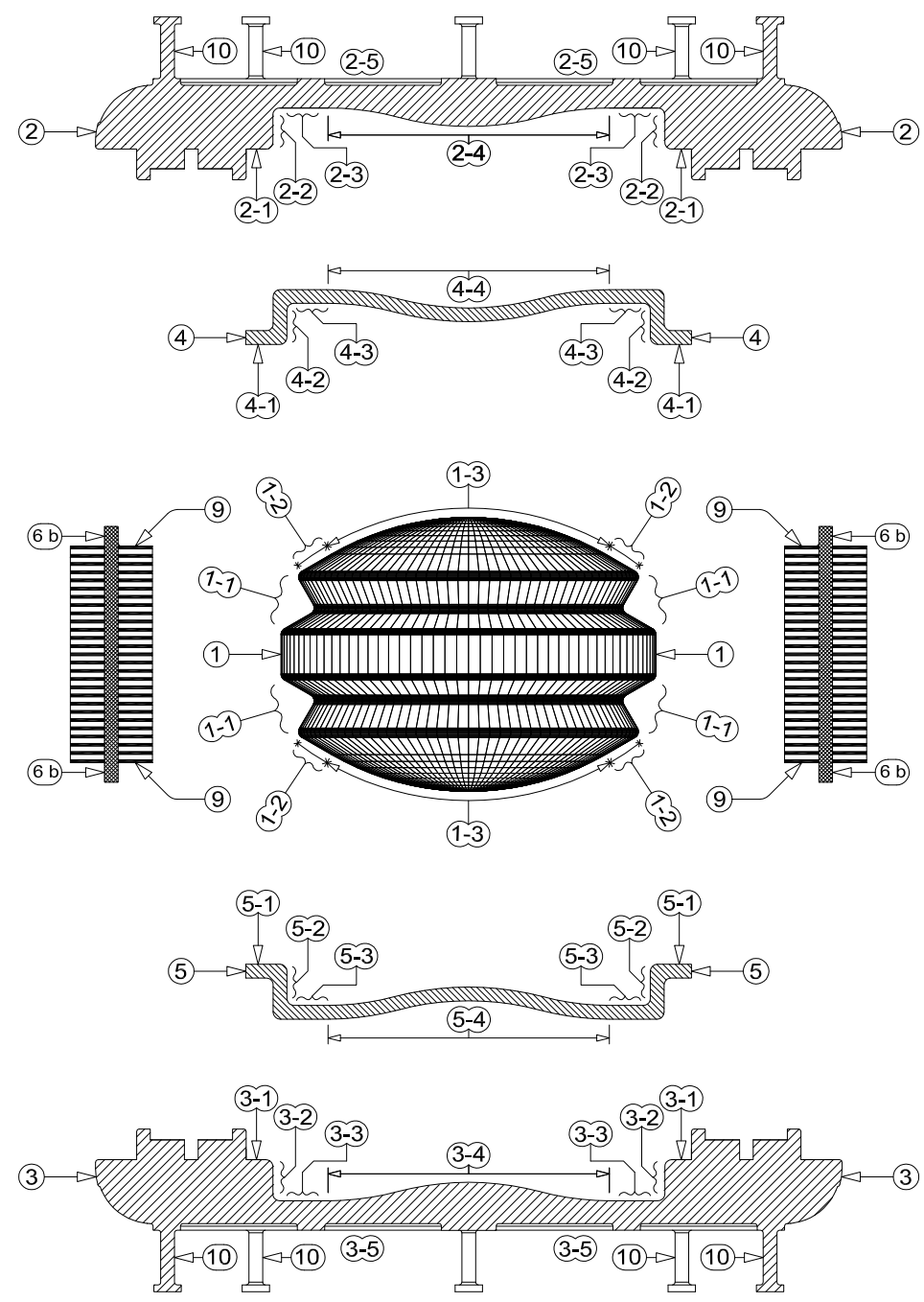

Figure 3.7: Details of the RNC type b, RNC-b

elliptical rolling body (1) as illustrated by Fig. 3.11. Regarding the RNC-a and RNC-b, the main supporting system of vertical loads is the hollow elastomeric cylinder (9) that permits much higher structural weight to be supported, as shown in Fig. 3.2, 3.6, 3.3, 3.7. It is worthwhile to mention that the maximum vertical load capacity of the RNC isolator, types RNC-a and RNC-b, is calculated according to the overlapped areas between the topmost and the lowermost surfaces of the hollow elastomeric cylinder, at the extreme deformed position, as demonstrated by solid hatched areas in Fig. 3.12(a,d and c,f) with 


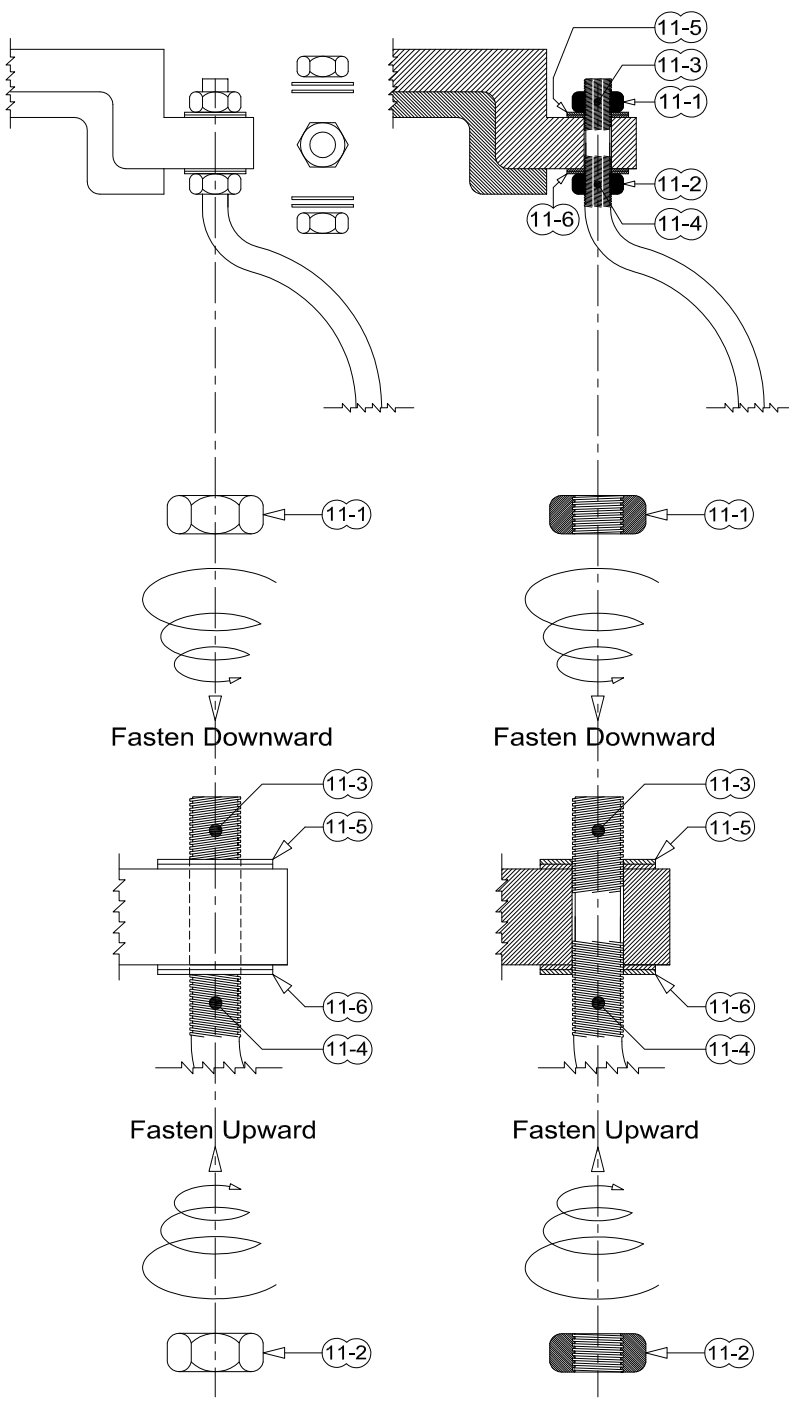

Figure 3.8: A proposed connection between metallic dampers and bearing plates for RNC-a and RNC-c, detail 11

no fear at all of buckling or p- $\Delta$ failure of the bearing.

In brief, the main feature of the RNC isolation system is that it allows great structure-base decoupling during earthquake while keeping enough resistance to minor excitations, has a built-in buffer, exhibits damping and uplift resistance. Then, returns back to its neutral position before excitation. 


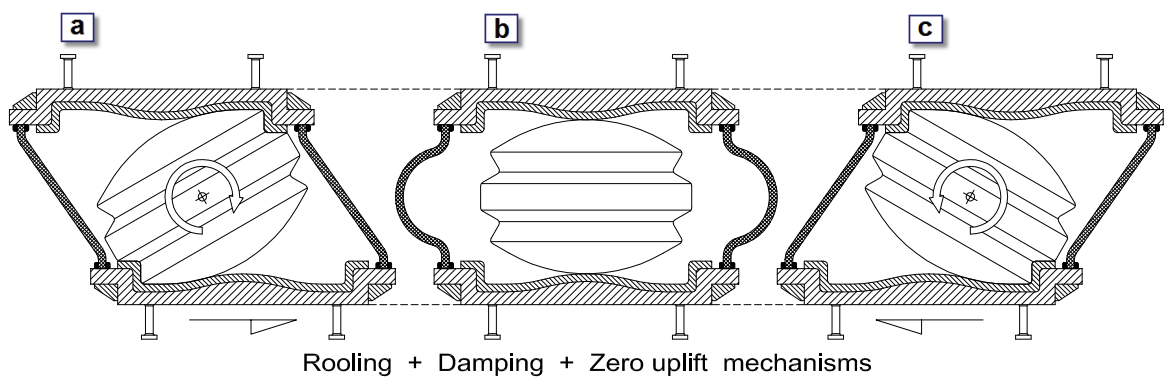

Figure 3.9: Rolling, damping and zero uplift mechanisms

\subsection{Component-mechanism relationship}

The appeal and potential of the RNC bearing are not only being a simple passive device, but also it is based on technologies and principles that are universally accepted in practice, just applied in an innovative manner. This section relates each component of the RNC isolator to its intended function/s in the whole assembled RNC isolation device. It is worthwhile to mention that many individual components are designed to serve for more than one purpose with the aim of simplicity and getting a relatively optimum compact design. In what follows, the components and their functions are categorized according to the main mechanisms included in the RNC isolation device. It is necessary to refer to Figs. 3.5-3.7 together with the reference figures in the next items.

\subsubsection{Rolling mechanism}

Rolling is the motion mechanism that the RNC isolator adopts to decouple the superstructure from its base. This sufficiently lengthens the period of vibration of the total system to reduce the seismic force response. The following components govern the rolling of the RNC isolator:

- The rolling body (1) through regular spherical surfaces (1-3) that are coarse enough to improve the friction and consequently force rolling motion instead of sliding motion.

- The deformable less stiff plates $(4,5)$ help magnifying the sliding friction coefficient with the rolling body and allow for smooth and comfort rolling. Further, these deformable plates $(4,5)$ help alleviating flattening, which increase the required force to initiate rolling, at contact points if the stiff plates $(2,3)$ and the stiff body (1) are in direct contact.

- The upper and lower bearing plates $(2,3)$ provide a suitable platform for rolling. Moreover, the curvatures $(2-4,3-4)$ makes rolling of the elliptical 


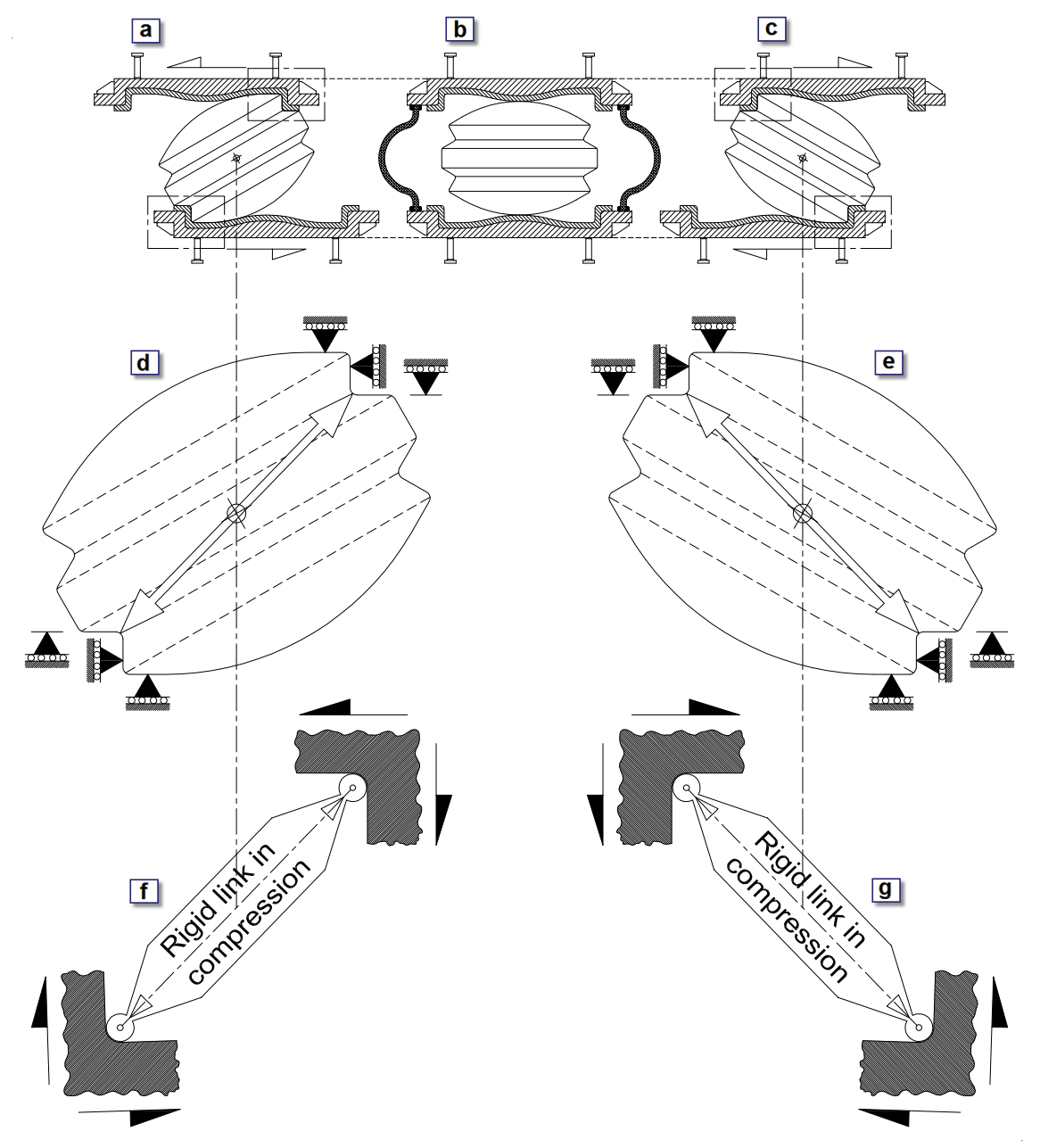

Figure 3.10: Buffer mechanism

roller exactly similar to rolling of the spherical roller, in the sense that elliptical roller consumes some energy to elevate the supported structure or object at the end of stroke. This forces the isolated system to vibrate as a pendulum having a limited vibration period, which represents a severe practical difficulty. Such limitation does not exist at all in spherical rollers and the RNC isolator as well, as shown in Fig. 3.9, thanks to curvatures $(2-4,3-4)$. 

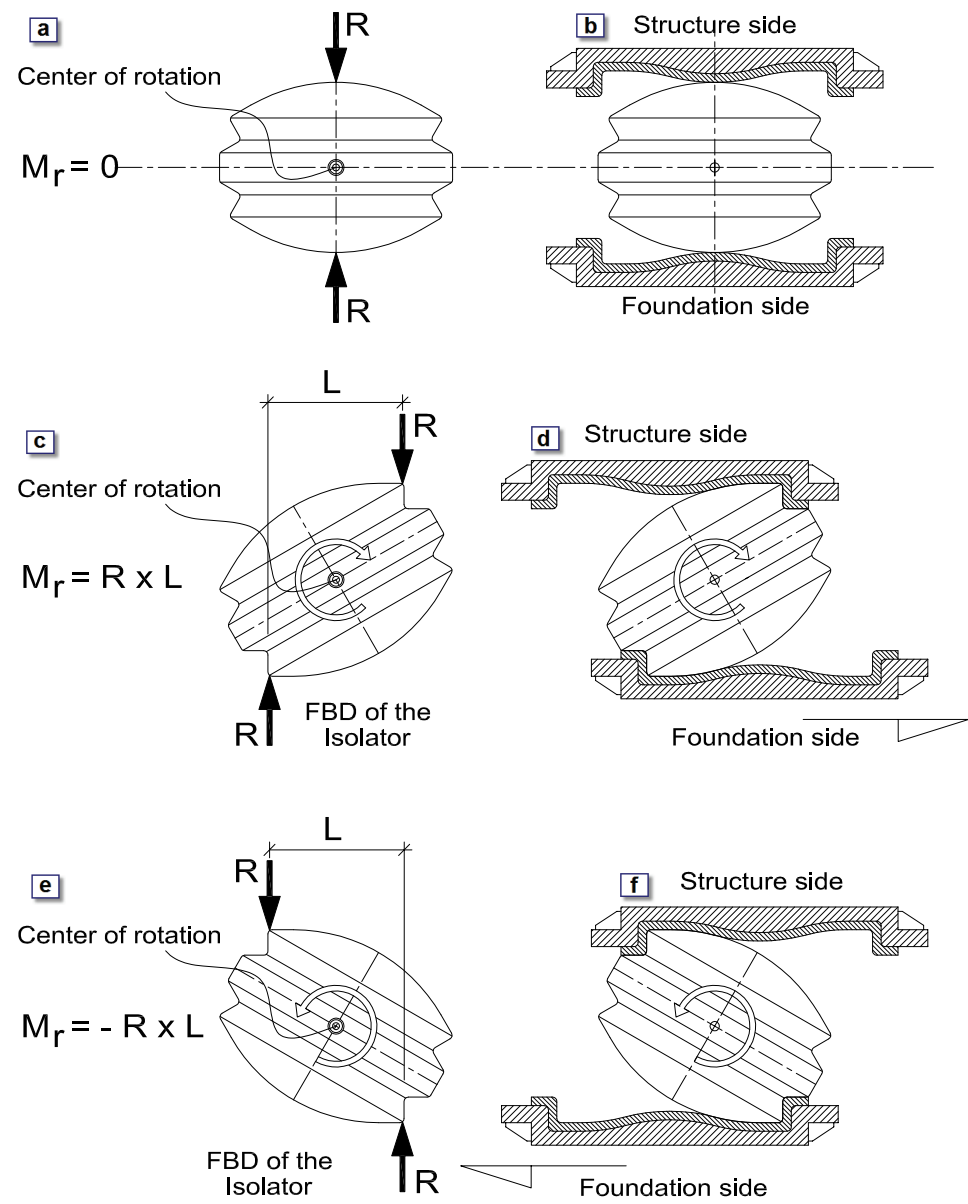

Figure 3.11: Gravity-based recentering mechanism

\subsubsection{Damping mechanism}

Metallic yield dampers are one of the most effective means of providing a substantial level of damping through hysteretic energy dissipation. These dampers are inexpensive and can provide a wide range of damping. Moreover, they are practical and widely available. So, they have been incorporated into the RNC isolator to control the relative deflections between isolated structure and ground to a practical design level. The following issues have been considered during the design of metallic yield dampers of the RNC isolator:

- To yield just after the seismic forces exceed the designed resistance to wind or minor vibrations. 

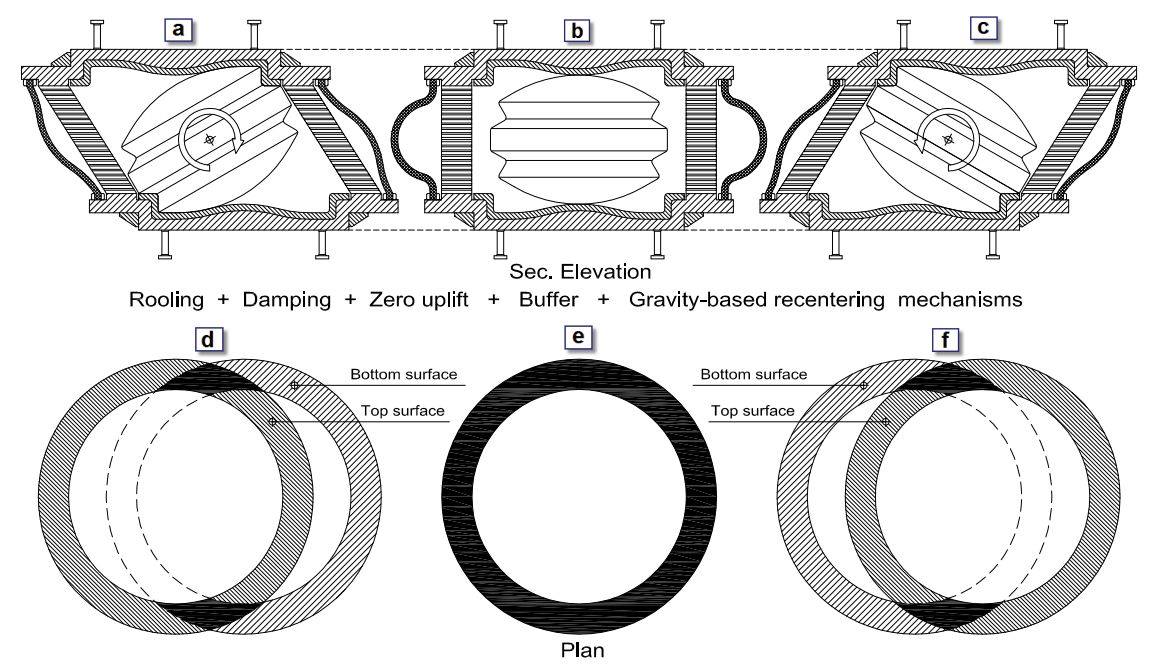

The common bearing area between top and bottom surfaces of the hollow Elastomeric cylinder

Figure 3.12: The main design bearing area of RNC-a and RNC-b

- To be long enough to allow the rolling body (1) to reach the edge buffer.

- To avoid sharp bends to reduce or even prevent stress concentrations at that bends.

- To exhibit the same shear strain in any horizontal direction.

- Never touch the rolling body (1) along the excitation duration.

- At the extreme deformed position, they should still have some outer curvature, i.e. away from the rolling body. This enables the metallic yield dampers to almost restore their initial shapes before deformation to start a new rolling stroke, see Figs. 3.12(a,c).

\subsubsection{Bearing mechanism}

The pointwise contact in the case of roller bearings reduces their ability to support heavier loads without flattening at the contact points. This main drawback has been alleviated in the $\mathrm{RNC}$ isolator whose bearing mechanism is improved by the following:

- Large radius of curvature of the rolling surfaces (1-3).

- Insertion of deformable less stiff (but strong, such as of neoprene material) plates $(4,5)$ between the rolling body (1) and the upper and lower stiff bearing plates $(2,3)$. This ensures getting contact areas instead of contact 
points. In addition, the less stiff plates $(4,5)$ prevent stiff-to-stiff pointwise contact between the stiff rolling body (1) and the stiff bearing plates $(2,3)$. Certainly, this avoids flattening of the stiff materials at the contact points under long-term vertical loading.

- The spherical rolling surfaces (1-3) are surrounded with plane surfaces (12 ) to substitute the reduction in the bearing area at the extreme displaced position, Fig. 3.9(a,c). Further, and with the aim of coincidence, the edge troughs of the upper $(2,4)$ and the lower $(3,5)$ plates are provided with similar plane parts $(2-2,2-3,4-2,4-3)$ and (3-2, 3-3, 5-2, 5-3), respectively.

- For more heavier vertical loads, the RNC isolator is provided with a hollow reinforced elastomeric cylinder (9), around the rolling body (1), of designed in-plan thickness to suit the anticipated vertical weights. This works as a main bearing mechanism in this case, while the rolling body itself acts as a secondary bearing mechanism. The design bearing area, of the elastomeric part, is the overlapped areas between upper and lower surfaces of the elastomeric cylinder at the extreme deformed position of the RNC isolator as shown in Fig. 3.12(a,d and c,f).

- Forming the elastomeric part as a hollow cylinder around the rolling body (1) allows for choosing any in-plan designed thickness without fear at all of buckling failure.

- In the case of elastomeric cylinder, the cumulative thickness of the upper and lower less stiff plates $(4,5)$ are taken equal to the cumulative thickness of rubber layers of the elastomeric cylinder. This insures better distribution of the vertical loads between the main and the secondary bearing mechanisms.

\subsubsection{Recentering mechanism}

Wherever possible, practical isolation systems should be designed to provide a restoring force to return the isolated structure (or object) to its original position after earthquake and to reduce or prevent torsion response. The RNC isolator is provided with an efficient gravity-based recentering mechanism through the elliptically shaped rolling body (1) along with the weight of supported structure or object. Such mechanism works as follows:

- At neutral position, the structural weight has the same line of action of its reaction. Therefore, the developed restoring moment $M_{\mathrm{r}}$ is zero, keeping steady situation.

- As the relative motion between the isolated structure and the ground initiates, an eccentricity or offset develops between the two vertical lines of action of the downward weight and its upward reaction. This eccentricity 
is equal to the developed relative displacement between structure and ground. Therefore, a restoring couple $M_{\mathrm{r}}$ is generated opposite to the motion-causing couple as illustrated by Fig. 3.11, so as to the two lines of actions of the structural weight and its reaction coincide again at zero restoring couple $M_{\mathrm{r}}$.

- The restoring moment $M_{\mathrm{r}}$ is directly proportional to the displacement amplitude, eccentricity of the rolling body and the structural, or object, weight.

\subsubsection{Buffer mechanism}

Isolators are normally designed to accommodate a travel distance greater than that which would occur during design earthquakes. However, during extreme low-probability earthquakes there is a possibility that the base of the structure will arrive at the end of the isolator design displacement when the structure still has considerable kinetic energy. If a stiff structure encounters a rigid base buffer with considerable kinetic energy, the ductility demand on the structure may be high, and may even substantially exceed the structure's design deformation capacity. The use of an energy-absorbing buffer can considerably decrease structure-base impact velocity. The RNC is provided with a rigid buffer covered with a deformable hyperelastic material, such as neoprene material, through the deformable less stiff plates $(4,5)$. In the RNC isolator, the following components collectively constitute the buffer mechanism as shown in Fig. 3.10:

- The two right-angle grooves (1-1) in the stiff rolling body (1).

- The vertical side walls (2-2, 3-2) of the upper and lower stiff plates $(2,3)$, along with the flat horizontal parts $(2-1,2-3,3-1,3-3)$. These side walls along with the grooves mentioned in the previous item in addition to the structural weight make the rolling body works as a rigid link in compression, at the extreme deformed positions, in an opposite direction to motion, as illustrated by Figs. 3.10(f,g), to force the isolated structure to stop at that point.

- The less stiff deformable plates $(4,5)$ through the parts $(4-1,4-2,4-3,5-1$, $5-2,5-3)$ to attenuate the possible severe shocks during extreme earthquakes.

- The metallic yield dampers (6a) help reducing the rolling velocity of the rolling body (1) before reaching the extreme deformed position, where they suffer high tensile forces to stretch their curvatures at that position, see Figs. 3.9(a,c) and 3.12(a,c).

- The plain parts (1-2) of the rolling surfaces of the rolling body (1) also help reducing the rolling body velocity just before hitting the vertical side walls. 
Note: In this dissertation, the maximum rolling displacements of the RNC isolators during earthquakes were determined without using the built-in buffer (stoppers) mechanism, to check weather they are affordable or not. Then, the buffer is designed to allow for rolling displacements a little bit higher. During stronger earthquakes, the rolling displacement limits may be exceeded but the buffer prevents such excessive displacements with minimal shock. This last point has not been studied in this dissertation, because there is no available model, at the moment, for the built-in buffer mechanism of the RNC isolator. Such study is postponed to the near future experimental work.

\subsubsection{Uplift-restraining mechanism}

When seismic isolated structure is subjected to strong ground excitation, including near-fault effects, undesirable uplift (or tension) in the isolation bearings may be induced. In fact, a variety of conditions may contribute to the development of either tensile forces or uplift in isolation bearings. Typical examples include slender structures with large height-to-width aspect ratios, certain types of bridges with large ratios of height of the centroidal axis to the distance between the bearings, and buildings incorporating bearings below braced columns or stiff walls. Owing to its distinct configuration, the RNC isolator possesses a reasonably efficient mechanism to resist uplift or tension. The following components contribute establishing an uplift resistance mechanism in the RNC isolator:

- As the rolling motion necessitates very low force to initiates, the rocking motion and consequently the developed tension in the RNC bearing is unlikely to occur to some extent.

- If the RNC has to support tensile forces under any condition, the metallic yield dampers can support reasonable amount of tension.

- To prevent the vertical elevation of the RNC-supported structure (or object) during rolling, the upper and lower stiff plates $(2,3)$ are provided with triple curvatures $(2-4,3-4)$ that are carefully designed to exactly absorb the expected elevation during rolling of the elliptical rolling body (1), over the full time history of excitation, as seen in Fig. 3.9.

\subsubsection{Initial stiffness mechanism}

Practical isolation systems usually offer adequate resistance to minor excitations, to guarantee structural stability and human comfort under service loads. The RNC isolator is provided with some different mechanisms to achieve sufficient resistance under low-level excitations: 
- Metallic dampers, which provide adequate initial (elastic) stiffness.

- Eccentricity of the elliptical rolling body, as explained in Chapter 4.

- Reasonably high rolling friction coefficient between a coarse-surfaced rolling body (1) and a synthetic rubber material (neoprene).

- Rolling of the stiff body (1) on rubber material slows down the rolling speed, depending on the thickness of the rubber material.

\subsection{Conclusions}

An innovative rolling-based seismic isolation bearing, denoted as RNC isolator, is introduced in this chapter. Owing to its distinct configuration, the $\mathrm{RNC}$ isolator possesses unique properties for a seismic isolator. The properties that distinguish the $\mathrm{RNC}$ isolator from the conventional rolling-based isolators include:

- Energy dissipation.

- Uplift restraint.

- Resistance to wind and minor vibrations.

- Built-in buffer.

- Inherent gravity-based recentering mechanism.

- Enhanced bearing mechanism.

- Resistance to flattening of contact surfaces.

- Wide range of stiffness and damping.

- Independent damping and bearing mechanisms.

- Independent stiffness and bearing mechanisms.

- Non-fixed vibration period.

This chapter has concentrated primarily on introducing the device, its concepts and establishing the underlying principles of operation in a clear detailed manner. 


\section{Modeling and characterization}

\subsection{Introduction}

Mechanical characterization of a system or a device can be performed experimentally or via numerical simulation. The former approach is more costly than the later one, but helps getting physical understanding of the system and therefore it can be employed for final verification. In contrast, numerical simulation uses numerical methods to quantitatively represent the evolution of a physical system. By using accurate models, the result of such simulation can have a good representation of the real environment. This enables safe drawing of proper conclusions and getting a reasonable understanding of the system.

In this dissertation, the numerical simulation is adopted to allow fully identification of the $\mathrm{RNC}^{1}$ isolator mechanical characteristics before its construction. A general scheme is presented in this chapter for that purpose through subjecting the RNC isolator to simultaneous horizontal and vertical loads as in typical practical situations. Further, a mathematical description of the main features associated to rolling of the $\mathrm{RNC}$ isolator is presented, and an input-output mathematical model is obtained to describe in a reasonable and manageable form the force-displacement relationship exhibited by the RNC isolator.

\footnotetext{
${ }^{1}$ The term RNC isolator refers generically to any of the forms described in Section 3.4. When referring to a particular RNC isolator form, the terms $\mathrm{RNC}-\mathrm{a}, \mathrm{RNC}-\mathrm{b}$ or $\mathrm{RNC}-\mathrm{c}$ are used instead.
} 


\subsection{Mechanical characterization}

The general-purpose finite element code ANSYS Multiphysics [4] is used to enable computer-aided design and testing of the RNC isolation system. For a desired configuration of the RNC system, the following steps are followed ${ }^{2}$ :

- Design and modeling of the individual components of the RNC isolation system: rolling body (1), upper and lower stiff bearing plates $(2,3)$ and less stiff plates $(4,5)$, metallic yield dampers (6), elastomeric cylinder (9) and bearing plate stiffeners $(7,8)$.

- Assembly of the individual components to set up the whole isolator and identification of all contact conditions among them.

- Definition and assignment of materials for each individual component.

- Selection of appropriate finite element type and mesh size according to the expected behavior of the component materials.

- Assignment of boundary conditions through nodal constraints and nodal restraints.

- Static application of the vertical structural weight and running full nonlinear analysis to capture all nonlinearities arising from the load application.

- Dynamic application of the horizontal ground motion starting from the last load step in the previous static analysis and running of full nonlinear transient dynamic analysis.

- Analysis of the results.

Through this scheme, a real scale model is designed. An extensive and detailed series of tests is carried out in a machine-like environment, which accurately simulates the response of the device subjected to a real testing machine. This allows fully identification of the RNC mechanical characteristics before its construction. In the machine-like testing, the topmost surface of the modeled RNC unit is allowed to move vertically (without rotation) under the structure own weight but it is always kept fixed in horizontal direction. The lowermost surface of the RNC unit is kept always fixed in the vertical direction. In horizontal direction, it is kept fixed only during the vertical application of the structure own weight. When the ground motion is applied, the lowermost surface is released horizontally without rotation. The less stiff plates $(4,5)$ are completely glued to the inner surfaces of the upper and lower steel bearing plates $(2,3)$, respectively, while they are kept in rolling contact with the rolling body (1).

\footnotetext{
${ }^{2}$ The different elements of the RNC isolator are numbered according to Figs. 3.5-3.8 in Chapter 3.
} 
In this dissertation, the rolling body (1), top stiff plate (2), lower stiff plate (3) and stiffeners $(7,8)$ are made of steel. The less stiff plates $(4,5)$ are made of neoprene material. The hollow elastomeric cylinder is designed according to [3] using method B for steel-reinforced elastomeric bearings. The metallic yield dampers are made of mild steel and are replaced with the embedded lead bars that are used to provide damping in the case od RNC-b.

\subsubsection{Types of tests}

The designed real-scale model of the $\mathrm{RNC}$ bearing is tested in the machine-like environment by subjecting the bearing to simultaneous horizontal and vertical dynamic loading. The maximum mesh element size of the nonlinear parts is $0.005 \mathrm{~m}$ for better accuracy and robust nonlinear analysis.

\section{Basic test}

The basic type of test is a sinusoidal horizontal displacement-controlled loading, conducted at the design vertical load, and for 5 cycles of loading at shear strain amplitudes of $5 \%, 10 \%$, and $20 \%$ that correspond to $25.4 \mathrm{~mm}, 50.8 \mathrm{~mm}$, and $101.6 \mathrm{~mm}$, respectively. The frequency of loading is $1 \mathrm{~Hz}$ for all of these tests.

\section{Variable axial load test}

The basic test is also performed at a number of different axial loads. The load is constant during each test, and tests are performed at loads of $2 P_{\text {des }}, P_{\text {des }}$, and $0.5 P_{\text {des }}$, where $P_{\text {des }}$ represents the design axial load. The rate of loading and the strain increments are the same as for the basic test described above.

\section{Variable frequency test}

The basic test is also performed at loading frequencies of $0.2,0.3,0.5,0.7,1.1$, $1.3,1.7,1.9,2.3$, and $2.9 \mathrm{~Hz}$. The other test variables are the same as for the basic test.

\subsubsection{Nonlinear modeling}

Structural nonlinearities arise from a number of causes, which can be grouped into these principal categories:

- Contact

Contact problems are highly nonlinear since they are status-dependent. 
This type of nonlinearity is encountered in the proposed RNC isolator as a result of steel-neoprene contact.

- Geometric nonlinearity

It is mainly a result of large strain and refers to the nonlinearities in the structure or component due to the changing geometry as it deflects. Due to low stiffness and severe ground shaking, the proposed RNC isolator experiences large horizontal deformation causing it to respond nonlinearly.

- Material nonlinearity

Material nonlinearities are due to the nonlinear relationship between the stress and strain, that is, the stress is a nonlinear function of the strain. The relationship is also path dependent (except for the case of nonlinear elasticity and hyperelasticity), so the stress depends on the strain history as well as the strain itself. This class of nonlinearity exists in the proposed $\mathrm{RNC}$ isolator due to the hyperelastic behavior of neoprene and the plastic behavior of both mild steel and lead dampers.

\section{Hyperelasticity}

Hyperelasticity refers to materials which can experience large elastic strain that is recoverable. Rubber-like and many other polymer materials fall in this category. The constitutive behavior of hyperelastic materials is usually derived from the strain energy potentials. Also, hyperelastic materials generally have very small compressibility. This is often referred to incompressibility. The hyperelastic material models assume that materials response is isotropic and isothermal and also assumed to be nearly or purely incompressible. Material thermal expansion is also assumed to be isotropic.

The hyperelastic behavior of the elastomeric neoprene pads is modeled using the 2-parameter Mooney-Rivlin model [4], which has an applicable strain of about $100 \%$ in tension and $30 \%$ in compression and in which the form of the strain energy potential, $E_{\mathrm{sp}}$, is

$$
E_{\mathrm{sp}}=c_{10}\left(\bar{I}_{1}-3\right)+c_{01}\left(\bar{I}_{2}-3\right)+\frac{1}{d}(J-1)^{2},
$$

where $\bar{I}_{1}$ is the first deviatoric strain invariant; $\bar{I}_{2}$ is the second deviatoric strain invariant; $c_{10}, c_{01}$ are constants characterizing the deviatoric deformation of the material; $d$ is the material incompressibility parameter. The initial shear modulus is defined as $\mu=2\left(c_{10}+c_{01}\right)$, and the initial bulk modulus is defined as $K=\frac{2}{d}$, where $d=(1-2 \nu) /\left(c_{10}+c_{01}\right)$. In this study, the hyperelastic material is the neoprene of the less stiff plates and the hollow elastomeric cylinder, which has the following characteristics: $c_{10}=38.25 \mathrm{t} / \mathrm{m}^{2}, c_{01}=9.56 \mathrm{t} / \mathrm{m}^{2}$, $d=2.09 \times 10^{-5}$ and $\mu=2\left(c_{10}+c_{01}\right)=95.62 \mathrm{t} / \mathrm{m}^{2}$. 


\section{Plasticity}

Plasticity is a nonconservative, path-dependent phenomenon. Plastic behavior, characterized by nonrecoverable strain, begins when stresses exceed the material's yield point. In other words, the sequence in which loads are applied and in which plastic responses occur affects the final solution results. Plastic response is anticipated in the isolator analysis as a consequence of employing the mild steel in RNC-a and $\mathrm{RNC}^{-} \mathrm{c}$ isolators or lead bars in $\mathrm{RNC}-\mathrm{b}$ as energy dissipation mechanisms.

The plastic behavior of the mild steel and lead bars is represented using the Bilinear Kinematic Hardening [4]. This option assumes the total stress range is equal to twice the yield stress, so that the Bauschinger effect is included. The material behavior is described by a bilinear total stress-total strain curve starting at the origin and with positive stress and strain values. The initial slope of the curve is taken as the elastic modulus of the material $E$. At the specified yield stress $C_{1}$, the curve continues along the second slope defined by the tangent modulus $C_{2}$. In this study, the mechanical characteristics of mild steel are: $E=2.1 \times 10^{7} \mathrm{t} / \mathrm{m}^{2}, C_{1}=24000 \mathrm{t} / \mathrm{m}^{2}, C_{2}=1.0353 \times 10^{5} \mathrm{t} / \mathrm{m}^{2}$ and poisson ratio $\nu=0.29$. The lead material has the following characteristics: $E=1.41 \times 10^{6} \mathrm{t} / \mathrm{m}^{2}, C_{1}=703.1 \mathrm{t} / \mathrm{m}^{2}, C_{2}=1138 \mathrm{t} / \mathrm{m}^{2}$ and poisson ratio $\nu=0.42$.

\section{Contact analysis}

Contact problems are highly nonlinear and require significant computer resources to solve. It is important to understand the physics of the problem and take the time to set the model to run as efficiently as possible. Contact problems present two significant difficulties. First, one generally do not know the regions of contact until the problem is run. Depending on the loads, material, boundary conditions, and other factors, surfaces can come into and go out of contact with each other in a largely unpredictable and abrupt manner. Second, most contact problems need to account for friction. There are several friction laws and models to choose from, and all are nonlinear. Frictional response can be chaotic, making solution convergence difficult.

In this study, the inner surfaces of the neoprene plates $(4,5)$ and the outer surfaces of the rolling body (1) are always kept in rolling contact using surfaceto-surface contact during the performed full nonlinear analysis. In the ANSYS model, the contact pair is formed by a target and a contact surfaces. The target (rigid) surface can penetrate the contact (deformable) surface but the later can not penetrate the earlier. So, the outer surfaces of the rolling body (1) are rigid and consequently they are assigned to the target surfaces of contact pairs, while the inner surfaces of the neoprene plates $(4,5)$ are assigned to the contact 
surfaces of the contact pairs as they are deformable in order to approach the realistic behavior of the assembled components.

In the ANSYS model, deformable-deformable contact pairs, between the stiff rolling body and the deformable neoprene plates of the RNC isolator, are identified using the options listed in Table 4.1.

\begin{tabular}{ll}
\hline Contact algorithm & $:$ Augmented Lagrange method \\
Contact detection at & $:$ Gauss integration point \\
Contact stiffness factor FKN & $: 0.11387 \mathrm{E}+08 \mathrm{kN} \mathrm{m}^{-1}$ \\
The resulting contact stiffness & $: 0.10000$ \\
Penetration tolerance factor FTOLN & $: 0.50383 \mathrm{E}-03 \mathrm{~m}$ \\
The resulting penetration tolerance & $: 0.50000$ \\
Max. initial friction coefficient MU & $: 1.0000$ \\
Default tangent stiffness factor FKT & $: 0.10000 \mathrm{E}-01$ \\
Default elastic slip factor SLTOL & $: 0.49371 \mathrm{E}-04$ \\
The resulting elastic slip & $:$ Yes \\
Update contact stiffness at each iteration & $: 0.10000 \mathrm{E}+22 \mathrm{kN} \mathrm{m}{ }^{-2}$ \\
Max. friction stress TAUMAX & $: 0.49371 \mathrm{E}-02 \mathrm{~m}$ \\
Average contact surface length & $: 0.50383 \mathrm{E}-02 \mathrm{~m}$ \\
Average contact pair depth & $: 2.0000$ \\
Default pinball region factor PINB & $: 0.10077 \mathrm{E}-01 \mathrm{~m}$ \\
The resulting pinball region & $: 0.47132 \mathrm{E}-05 \mathrm{~m}$ \\
Auto contact offset used to close gap & $:$ Yes \\
Initial penetration is excluded & \\
\hline
\end{tabular}

Table 4.1: Contact pairs properties of the ANSYS-modeled RNC isolator

The contact surfaces are initially modeled to be in the just-in-touch position, but minimum initial gap of $4.667 \mathrm{E}-06 \mathrm{~m}$ was detected between contact elements due to finite element meshing. However, the gap is closed due to initial adjustment of contact surfaces.

\subsubsection{Mechanical characteristics}

To determine the maximum vertical load capacity of the proposed RNC isolator, a trial and error procedure has been carried out using ANSYS such that the vertical strain in the neoprene plates $(4,5)$ does not exceed $30 \%$ as recommended by [3].

Figs. 4.1, 4.3, 4.5 and 4.2, 4.4, 4.6, respectively, show the overall behavior of the assembled RNC unit before and after the static application of the vertical own weight of the supported structure (or object), respectively. The meshing 


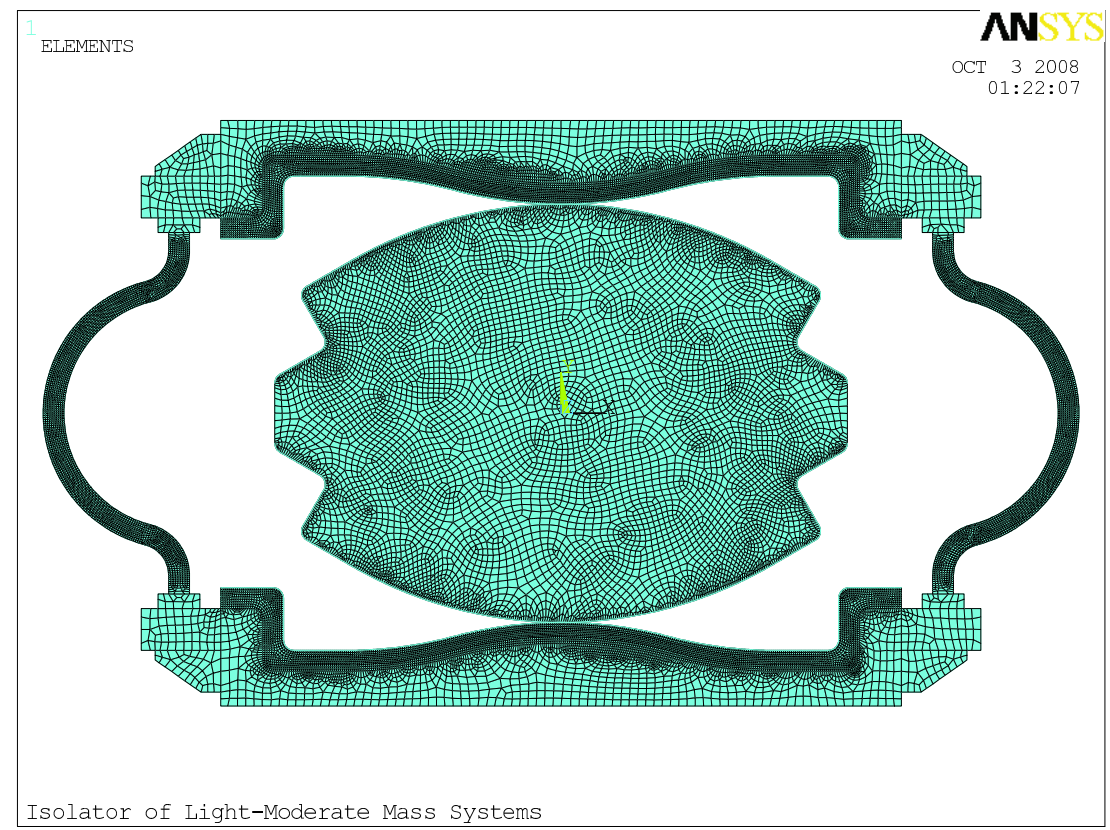

Figure 4.1: ANSYS finite element model, non-deformed shape, RNC-c.

is much more dense in components that exhibit nonlinear behavior (neoprene plates, metallic yield dampers and lead bars, rolling contact surfaces and elastomeric cylinder) if compared to other components that behave linearly (steel bearing plates, stiffeners and rolling body). Also, the behavior of the neoprene plates under loads seems to be realistic. Indeed, Figs. 4.2, 4.4, 4.6 show how these neoprene plates get deformed and how the corresponding contact areas with the rolling body increase, which translates into higher capacity to support vertical loads in addition to the vertical load supported by the hollow elastomeric bearing. Moreover, the RNC unit shows no lateral motion during this loading stage, in which the topmost surface exhibits the maximum vertical displacement (without rotation) contrary to the lowermost surface which is fixed vertically and exhibits no rotation.

Starting from the last load step of the performed static analysis, a sinusoidal horizontal displacement is applied at the lowermost surface of the RNC isolator, and the resulting force at the topmost surface is measured. The measured force-displacement relationship for the a RNC design is plotted using time and displacement scales, in dashed lines, as shown in Figs. 4.7(a,b) respectively. Then, an equivalent bilinear approximation of the measured hysteresis response $[401,333]$ is carried out as shown in solid lines in Figs. 4.7(a,b). From this bilinear force-displacement relationship, the following mechanical characteristics are obtained for the RNC isolator: 


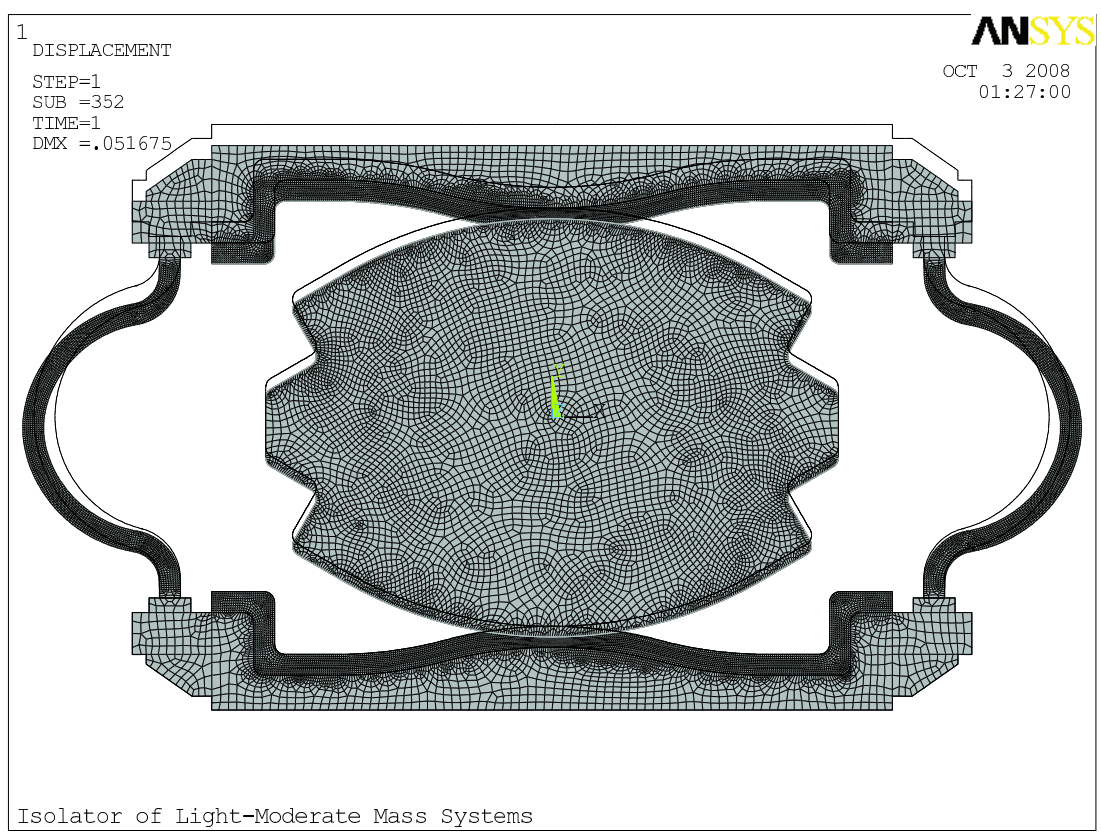

Figure 4.2: ANSYS finite element model, non-deformed + deformed shapes due to vertical structural weight, RNC-c.

1. Pre-yield stiffness.

2. Post-yield stiffness.

3. Yield displacement.

4. Yield force.

5. Characteristic strength.

The actual obtained values of these characteristics, for each RNC isolator type, are given in the corresponding chapters.

\subsection{Mechanics of the elliptical rolling body}

In this section, a mathematical description of the main features associated to rolling of the RNC isolator is presented. Consider the free body diagram of the elliptical rolling body between the base mass and the foundation, as shown in Figs. 4.8, 4.9. The proposed bearing can roll in one or more horizontal directions using (quasi-) elliptical rolling rods or (quasi-) ellipsoidal bearings, respectively, having the same cross-sectional configurations. Further, it is assumed that the rolling friction coefficient $\mu$ between the rolling body, the base 


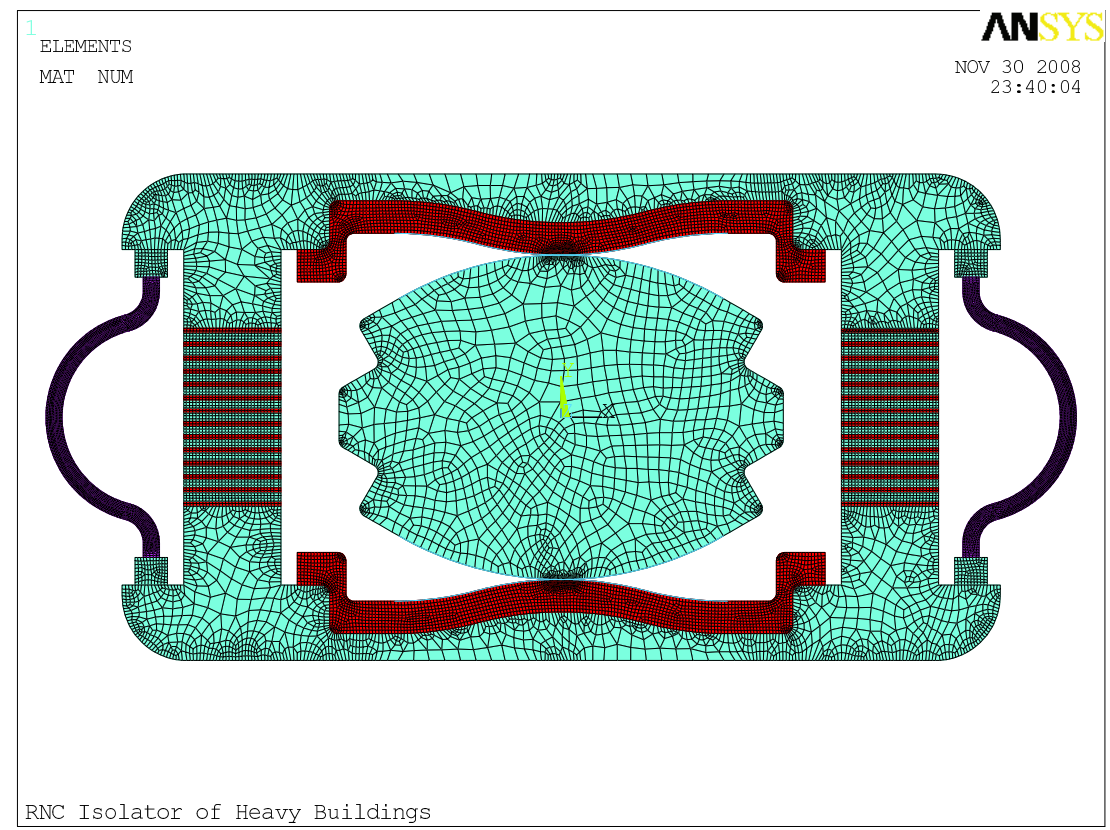

Figure 4.3: ANSYS finite element model, non-deformed shape, RNC-a.

mass and the foundation remains constant throughout the motion of the structure and is big enough to avoid any sliding between the rolling body and the foundation or base mass.

\subsubsection{Motion of the elliptical rolling body}

Let us consider a unidirectional motion of the proposed isolator in the horizontal $x$ axis. Although the rolling body is mainly formed by the intersection of two circles, the rolling surfaces fit perfectly with an ellipse having a major horizontal axis of $2 a$ and a minor vertical axis of length $2 b$ as shown in Fig. 4.8(a). The eccentricity of the elliptical rolling body is expressed as

$$
e=\sqrt{\frac{a^{2}-b^{2}}{a^{2}}} .
$$

The rolling body rolls in horizontal direction only during earthquake excitation, where the vertical uplift is avoided via the configuration of the inner surfaces of the upper and lower bearing plates, Figs. 3.1-3.7 and 3.9, 3.12(a,b,c) Chapter 3. Consider Figs. 4.8(a,b) where the elliptical rolling body is in rolled position between flat base mass and flat foundation. The motion is character- 


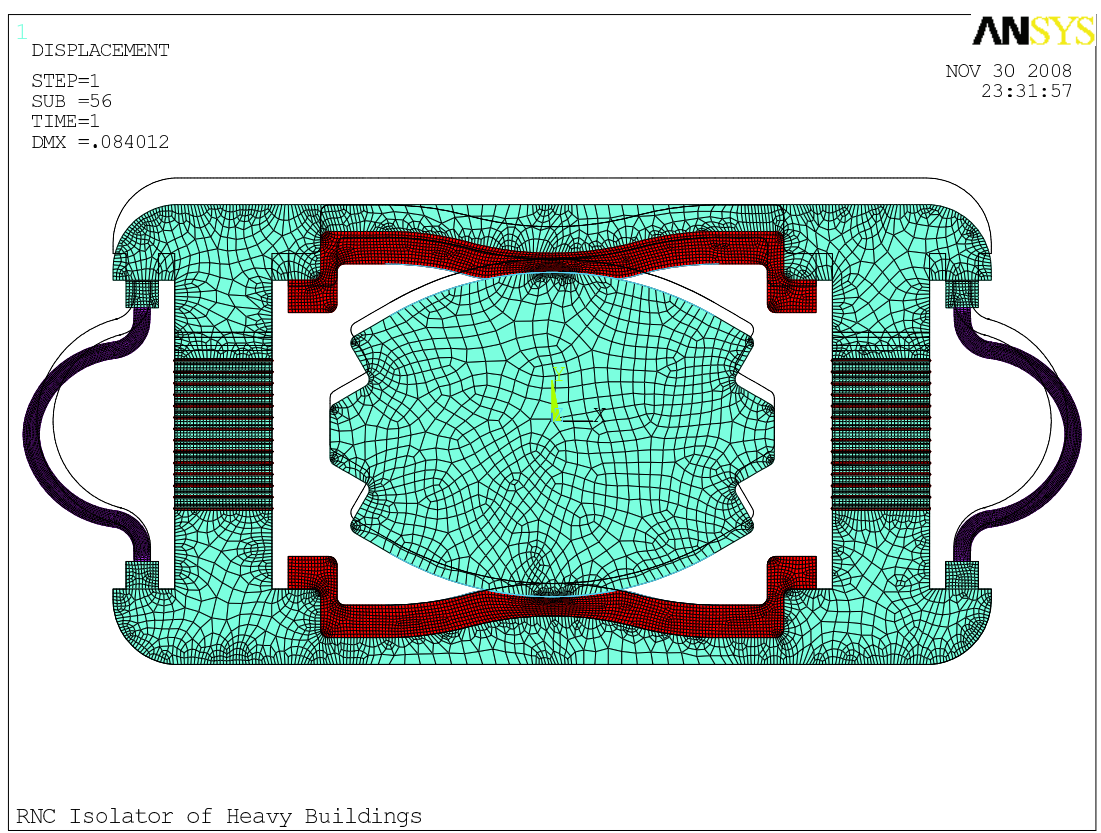

Figure 4.4: ANSYS finite element model, non-deformed + deformed shapes due to vertical structural weight, RNC-a.

ized by the following variables:

$$
\begin{gathered}
x_{r}=\frac{x_{b}}{2}, \\
y_{r}=p-b, \\
\theta_{r}=\tan ^{-1}\left(\frac{b}{a} \tan \theta\right),
\end{gathered}
$$

where $x_{r}$ and $y_{r}$ are the horizontal and vertical displacements of the center of gravity $(\mathrm{CG})$ relative to ground; $\theta_{r}$ is the rotation angle of the rolling body; $x_{b}$ is the horizontal displacement of the base mass relative to the ground; $p=$ $a \sin \theta \sin \theta_{r}+b \cos \theta \cos \theta_{r}$ is half the vertical distance between the lower and upper contact points [points A and B in Figs. 4.8(a)(b)] of the rolling body; $\theta$ is the eccentric angle such that the coordinates of point $\mathrm{A}$ are $(-a \sin \theta, b \cos \theta)$ and hence the distance $O A=\sqrt{a^{2} \sin ^{2} \theta+b^{2} \cos ^{2} \theta}=a \sqrt{1-e^{2} \cos ^{2} \theta}$.

By equating the distances $A A^{\prime}$ and $A A^{\prime \prime}$ in Fig. 4.8(b), a relation between the eccentric angle $\theta$ and the base displacement $x_{b}$ can be expressed as

$$
\frac{x_{b}}{2}+c=a \int_{0}^{\theta} \sqrt{1-e^{2} \cos ^{2} \phi} d \phi,
$$




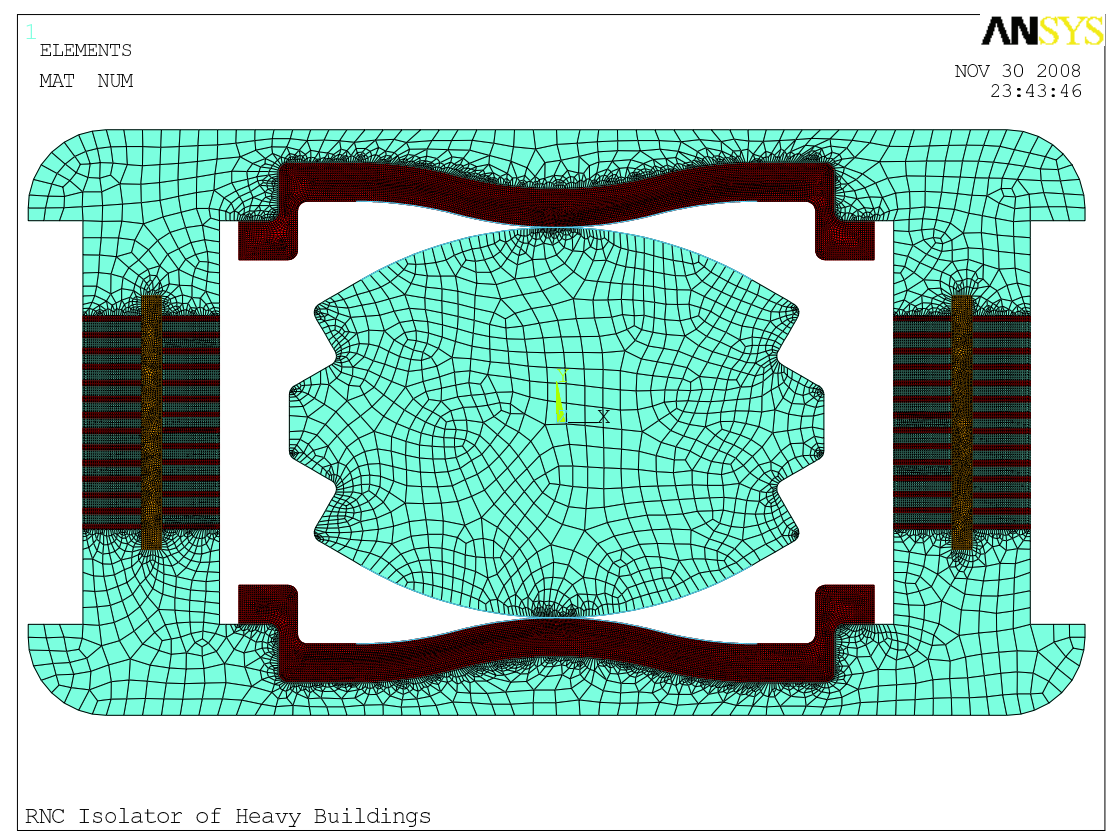

Figure 4.5: ANSYS finite element model, non-deformed shape, RNC-b.

where $c=a \sin \theta \cos \theta_{r}-b \cos \theta \sin \theta_{r}$ is half the horizontal distance between the upper and lower contact points. Note that the right hand side of Eq. (4.6) contains a special function known as an elliptic integral of the second kind. The value of this integral is available in standard charts and tables [521] knowing $e$ and $\theta$. Eq. (4.6) gives a design tool for the rolling body geometry. For a given value of $e$ and $\theta$, Eq. (4.6) allows to calculate the corresponding horizontal base displacement $x_{b}$.

\subsubsection{Restoring mechanism of the elliptical rolling body}

Considering the equilibrium of inertia forces acting on the rolling body, Fig. 4.9 , the horizontal restoring force $F_{\mathrm{b} 1}$ is expressed as

$$
F_{\mathrm{b} 1}=\frac{1}{2 p} J_{r} \ddot{\theta}_{r}+m_{r} \ddot{y}_{r} \frac{c}{p}+\frac{1}{2} m_{r}\left(\ddot{x}_{r}+\ddot{x}_{g}\right),
$$

where $m_{r}$ and $J_{r}$ are the mass and the moment of inertia of the rolling body and $\ddot{x}_{g}$ is the ground acceleration.

In the RNC isolator, as shown by Figs. 3.9, 3.12(a,b,c), the inner faces (facing the rolling body) of the upper and lower bearing plates are configured so that the vertical uplift of the supported object is inhibited along the whole 


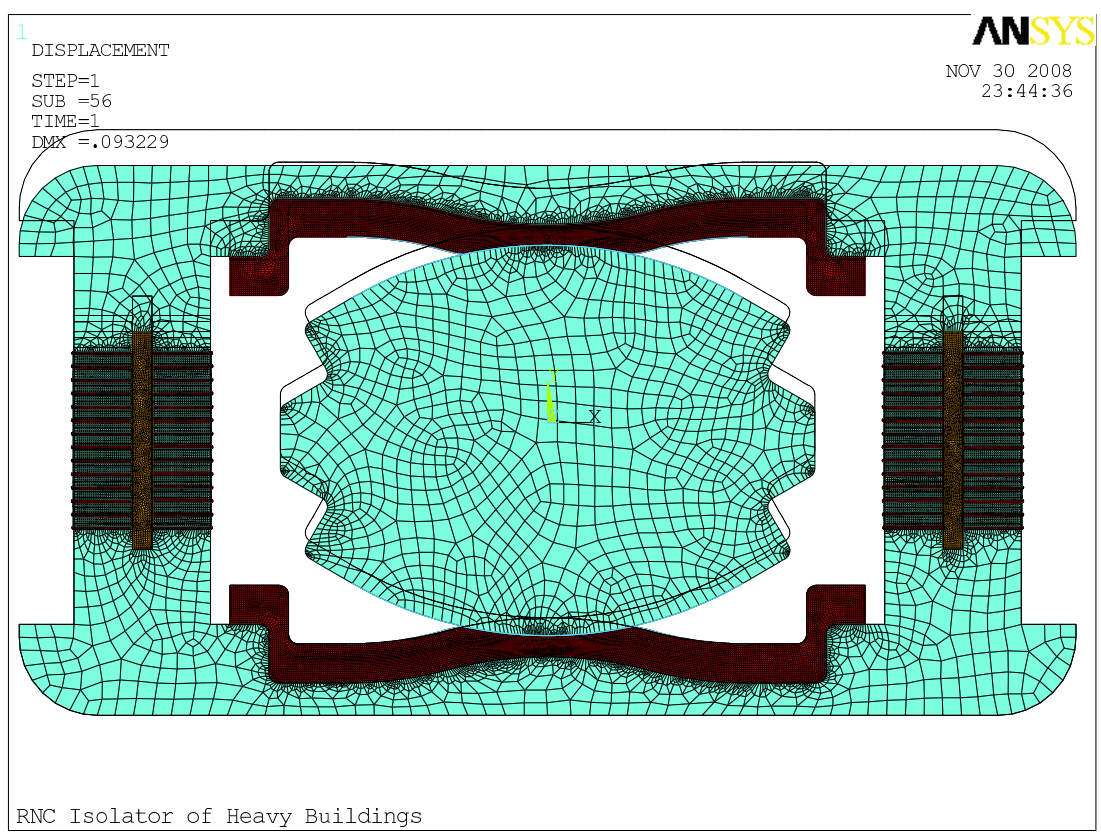

Figure 4.6: ANSYS finite element model, non-deformed + deformed shapes due to vertical structural weight, RNC-b.

time history of the excitation. This anti-uplift configuration is an inherent characteristic of the proposed RNC isolator. Accordingly, Eq. (4.4) becomes $y_{r}=p-b=0$ and Eq. (4.7) reduces to

$$
F_{\mathrm{b} 1}=\frac{1}{2 b} J_{r} \ddot{\theta}_{r}+\frac{1}{2} m_{r}\left(\ddot{x}_{r}+\ddot{x}_{g}\right) .
$$

The eccentricity of the elliptical rolling body $e$ provides a source of a gravity-based recentering mechanism to avoid permanent residual displacements of the superstructure after earthquake excitations. As shown in Figs. $4.8(\mathrm{~b}), 4.9$, the structural weight $W$ along with the eccentricity $e$ generate a recentering couple $M_{\mathrm{r}}$ of the value

$$
M_{\mathrm{r}}=W \times 2 c .
$$

As the eccentricity is always greater than zero for the rolling body, it is then considered an inherent characteristic of the RNC isolator. The recentering moment is maximum at the extreme displaced positions and is zero at the neutral position. 

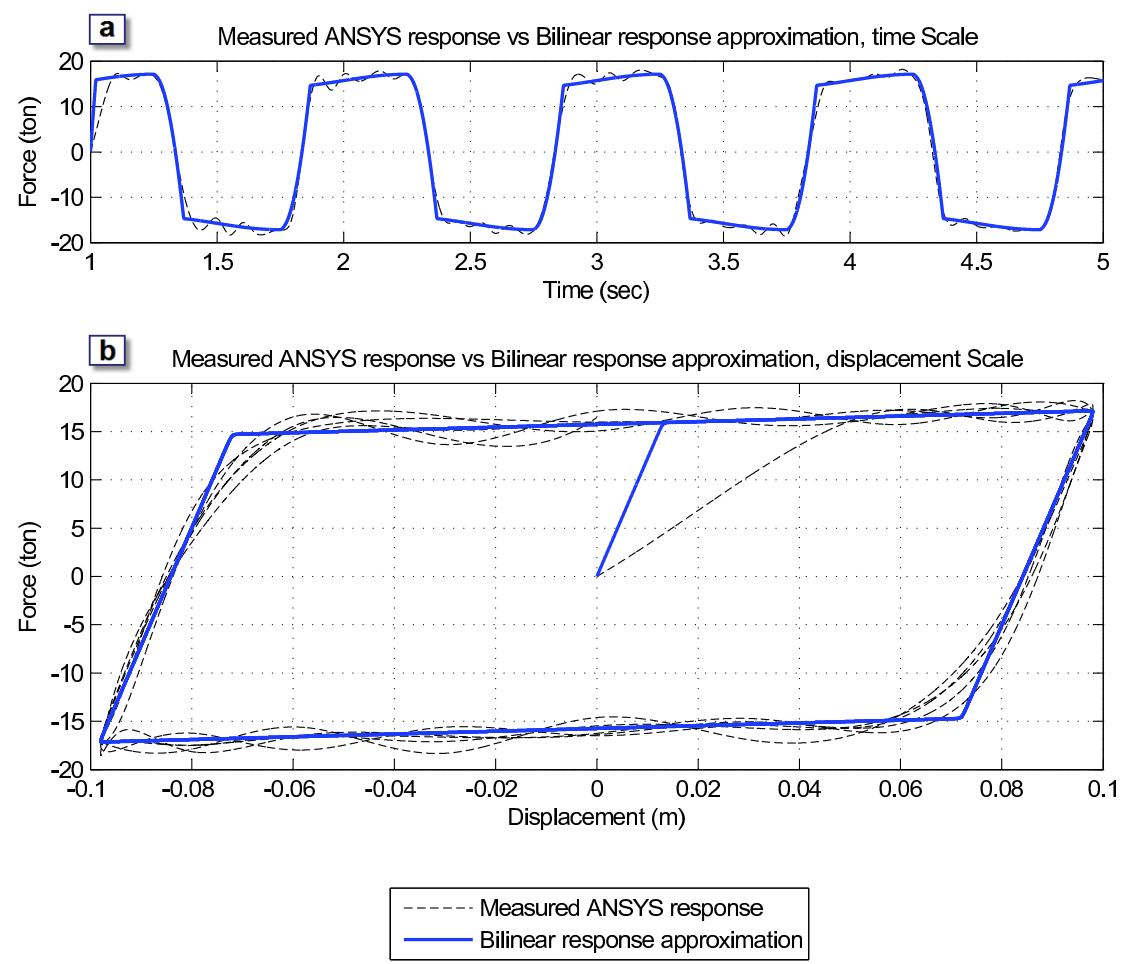

Figure 4.7: ANSYS measured response and its bilinear approximation: (a) Time scale, (b) Displacement scale.

\subsection{Resistance to minor vibrations}

Practical isolation systems usually offer adequate resistance to minor excitations, to guarantee structural stability and human comfort under service loads. The RNC isolator is provided with three different mechanisms to achieve sufficient resistance under low-level excitations:

1. Metallic dampers, which provide adequate initial stiffness.

2. Eccentricity of the elliptical rolling body.

3. Reasonably high rolling friction coefficient between a coarse-surfaced rolling body and a rubber material. The positive effect of rubber material compressibility on resisting rolling motion is neglected for both simplicity and more safety.

The system starts rolling just after the total horizontal force between the base mass and the rolling body exceeds the limiting value, provided by the 


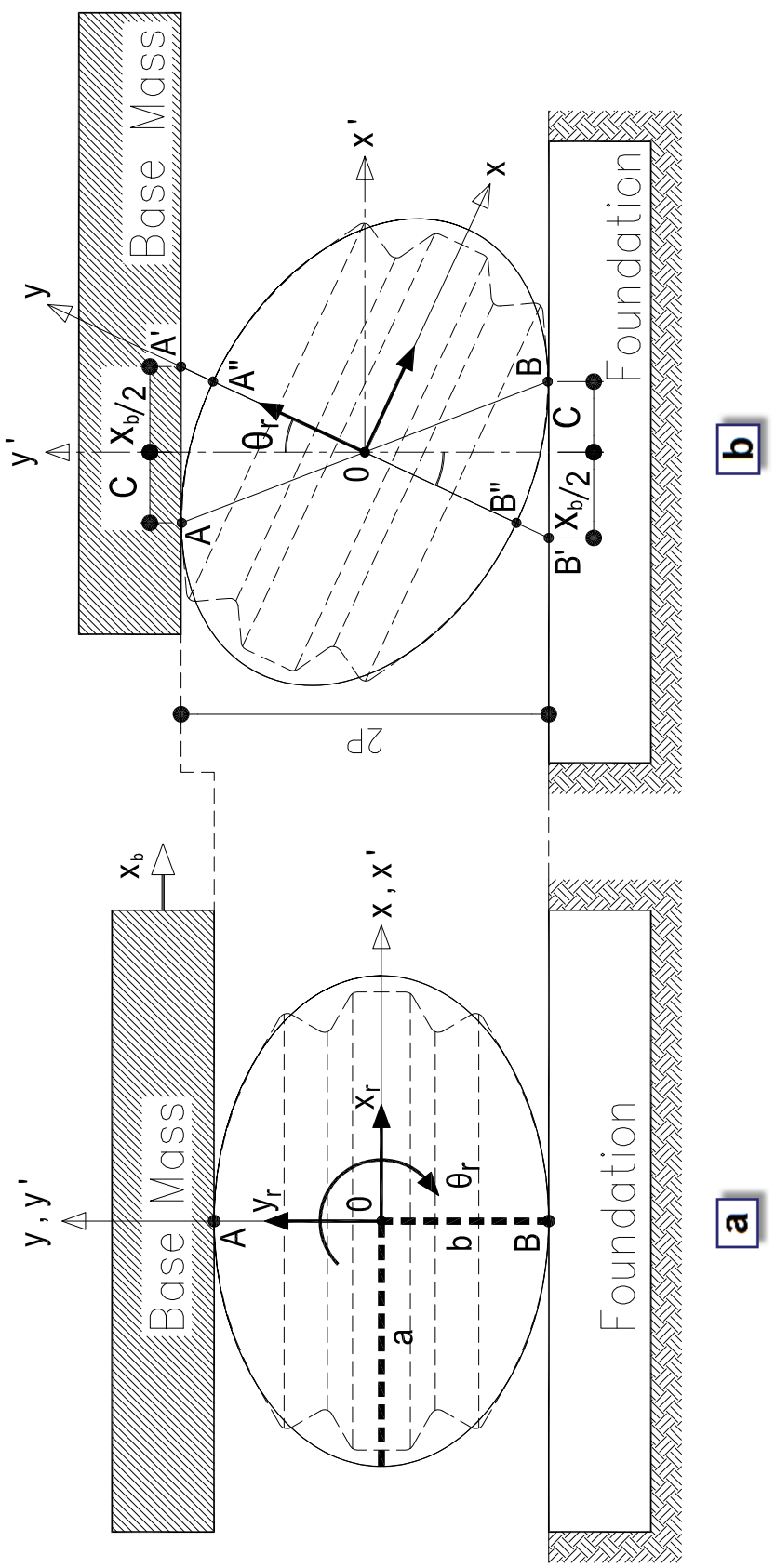

Figure 4.8: (a) The rolling body in neutral position; (b) The rolling body in deformed position. 


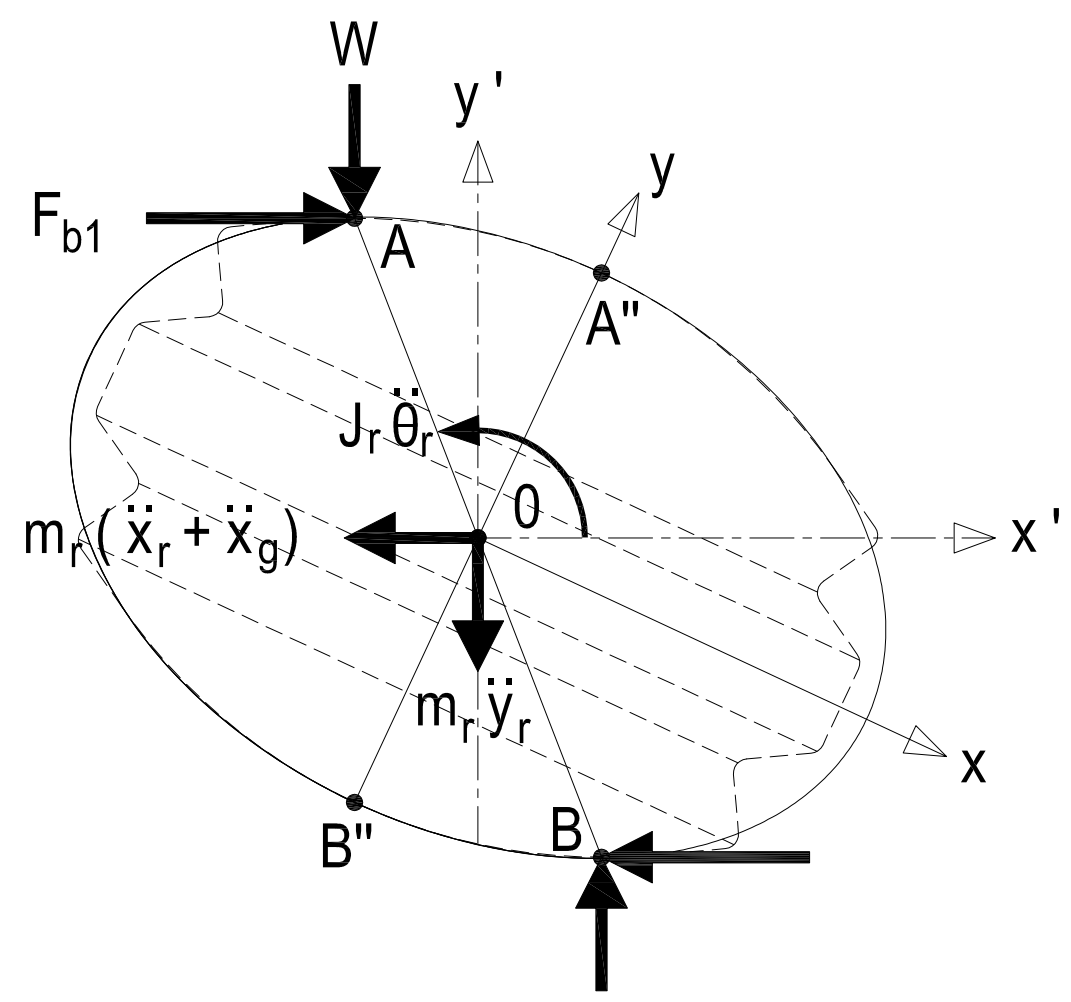

Figure 4.9: Free-body-diagran of the rolling body.

second and the third mechanisms, which is expressed as

$$
F_{\mathrm{LR}}=W\left(\mu \operatorname{sign}\left(\dot{x}_{b}\right)+\frac{c}{p}\right),
$$

where $W$ is the weight of the structure above the rolling body; $\mu$ is the coefficient of rolling friction between the base mass, the rolling body, and foundation; sign denotes the signum function, i.e. $\operatorname{sign}\left(\dot{x}_{b}\right)=\dot{x}_{b} /\left|\dot{x}_{b}\right|$; and $c, p$ have been defined in Section 4.3.1. In the expression of $F_{\mathrm{LR}}$, the first quantity inside the bracket is due to the rolling friction and the second quantity $c / p$ is due to the restoring force provided by the eccentricity of the elliptical rolling rods. Further, for the case of circular rolling body (i.e. $e=0$ and for which $c / p=0$ ), this expression reduces to that given by [291].

Just after rolling starts, lateral displacement gradually increases until the internal shear force exceeds the elastic limit $F_{\mathrm{LY}}$

$$
F_{\mathrm{LY}}=k_{e} d_{y}
$$

where $k_{e}$ is the elastic stiffness provided by metallic yield dampers and $d_{y}$ is the yield displacement (obtained in Section 4.2.3). In other words, the desired 
isolation effects take place when the total horizontal force exceeds the total limiting $F_{\mathrm{L}}$, which represents the maximum value of the $\mathrm{RNC}$ isolator resistance to wind or minor vibrations and is given by

$$
\begin{aligned}
F_{\mathrm{L}} & =F_{\mathrm{LR}}+F_{\mathrm{LY}}, \\
& =W\left(\mu \operatorname{sign}\left(\dot{x}_{b}\right)+\frac{c}{p}\right)+k_{e} d_{y} .
\end{aligned}
$$

\subsection{Hysteretic modeling of the energy dissipa- tion mechanism}

The characterization described in Section 4.2 has shown that the RNC isolator exhibits a hysteretic behavior, as shown in Fig. 4.7(b). The objective of this section is to obtain an input-output mathematical model to describe in a reasonable and manageable form the force-displacement relationship exhibited by the RNC isolator. The Bouc-Wen model of smooth hysteresis is considered.

\subsubsection{The Bouc-Wen model}

The Bouc-Wen model $[455,208]$ has been extensively used to describe nonlinear hysteretic behaviors, particularly, in seismic isolation devices. A thorough survey on that model is given in Appendix A. That survey is the first of its kind on the model since its origination more than 30 years ago. The BoucWen model appears in two forms in this dissertation, the standard and the normalized forms. The former form can be dealt with numerically to identify its parameters and it is overparametrized. The later (normalized) form provide exact analytical treatment for parameters identification and eliminates the overparametrization found in the standard form.

The motivation beyond using the standard form of the Bouc-Wen model is that all the response quantities of interest are obtained by means of a practical computer code SAP2000 advanced [6], which incorporates only the standard form of the Bouc-Wen model. While the normalized form of the model is used herein to capture three main advantages: (i) the warranty of a unique input/output behavior for each set of parameters which is ideal for identification purpose; (ii) the elimination of parameter redundancy or overparametrization; (iii) the availability of a robust parametric nonlinear, nonrecursive identification method of the normalized Bouc-Wen model [201, 194] based on an exact and explicit expression for the hysteretic limit cycle [200]. 


\subsubsection{The standard Bouc-Wen model form}

The standard form of the Bouc-Wen model is expressed as [455]

$$
\begin{aligned}
F_{\mathrm{b} 2}(t) & =\alpha k x(t)+(1-\alpha) D_{\mathrm{y}} k z(t), \\
\dot{z} & =D_{\mathrm{y}}^{-1}\left(A \dot{x}-\beta|\dot{x}||z|^{n-1} z-\gamma \dot{x}|z|^{n}\right),
\end{aligned}
$$

where $x$ is the displacement, $z$ is an auxiliary variable, $F_{\mathrm{b} 2}$ is the isolator restoring force, $\alpha k x$ is the elastic force component, $\dot{z}$ denotes the time derivative, $n>1$ is a parameter that governs the smoothness of the transition from elastic to plastic response, $D_{\mathrm{y}}>0$ is the yield constant displacement, $k>0$ and $0<\alpha<1$ represents the post to pre-yielding stiffness ratio $\left(k_{\mathrm{b}} / k_{\mathrm{e}}\right)$, while $A, \beta$ and $\gamma$ are non-dimensional parameters that govern the shape and size of the hysteresis loop.

The Bouc-Wen model can match a hysteretic behavior by properly tuning its parameters. It is a black-box model, i.e. the model parameters may not have physical meanings. For this reason, the Bouc-Wen model has to fulfill some general physical properties. It was asserted by [199] that, for $n \geq 1$, the Bouc-Wen model is bounded-input bounded-output (BIBO), passive, and consistent with physical asymptotic motion if and only if

$$
A>0, \quad \beta+\gamma>0, \quad \beta-\gamma \geq 0 .
$$

\subsubsection{Parameters estimation of the standard form}

By comparing the Bouc-Wen model output to the experimental (ANSYS) data, for a given periodic input displacement signal, the model parameters are estimated to characterize the RNC isolator. A total of 7 parameters $(A, \alpha, \beta, \gamma$, $D, n$, and $k$ ) are forced to obey the necessary conditions given in Section 4.5.2.

A constrained nonlinear least-squares optimization algorithm, available in MATLAB [5], is used to obtain the 7 model parameters in Eqs. (4.14) and (4.15). The optimized parameters are then determined to fit the model to the ANSYS output data.

\subsubsection{The normalized Bouc-Wen model form}

The normalized form of the Bouc-Wen model was proposed by [200] where the hysteretic restoring force is expresses as

$$
\begin{aligned}
F_{\mathrm{b} 2}(t) & =\kappa_{x} x(t)+\kappa_{w} w(t), \\
\dot{w}(t) & =\rho\left(\dot{x}-\sigma|\dot{x}||w|^{n-1} w-(\sigma-1) \dot{x}|w|^{n}\right),
\end{aligned}
$$


where $\kappa_{x}, \kappa_{w}, \rho, \sigma$ and $n$ are the shape controlling parameters of the hysteresis loop; and $w(t)$ is an auxiliary variable which is not accessible to measurement. Furthermore, and to guarantee BIBO stability, passivity, and consistency with physical asymptotic motion, the lower value of the parameter $\sigma$ is limited to $\frac{1}{2}$.

\subsubsection{Parameters estimation of the normalized form}

The normalized Bouc-Wen form provides an exact and explicit expression for the hysteretic limit cycle, [200]. Therefore, by using an input signal $x(t)$ as a periodic $T$-wave (see Fig. A.3) along with analytic description of limit cycle, a robust parametric nonlinear, nonrecursive identification method or the normalized Bouc-Wen model was presented by [201, 194]. This method provides exact values of the model parameters in the absence of disturbances, and gives a guaranteed relative error between the estimated parameters and the true ones in the presence of the perturbations. A detailed description of such method is given at the end of Section A.6.6, Appendix A. The relation between the normalized and the standard model parameters are given by Eq. (A.39).

\subsubsection{Verification of the Bouc-Wen model}

To check the validity of the identified parameters, an actual random seismic displacement is input into the ANSYS and the Bouc-Wen models. The discrepancy between the measured $F_{\mathrm{m}}$ (ANSYS) and the predicted $F_{\mathrm{b}}$ (Bouc-Wen model) outputs is quantified using the $L_{1}$ and $L_{\infty}$-norms and the corresponding relative errors $\varepsilon$ :

$$
\|f\|_{1}=\int_{0}^{T_{e}}|f(t)| \mathrm{d} t ; \quad\|f\|_{\infty}=\max _{t \in\left[0, T_{e}\right]}|f(t)| ; \quad \varepsilon_{1, \infty}=\frac{\left\|F_{\mathrm{m}}-F_{\mathrm{b}}\right\|_{1, \infty}}{\left\|F_{\mathrm{m}}\right\|_{1, \infty}} .
$$

The relative error $\varepsilon_{1}$ quantifies the ratio of the bounded area between the output curves to the area of the measured force along the excitation duration $T_{e}$, while $\varepsilon_{\infty}$ measures the relative deviation of the peak force.

The above mentioned models along with their parameter estimation and verification techniques are applied to different case studies, considering each $\mathrm{RNC}$ isolator type, in the in the next chapters.

\subsection{Conclusions}

In this chapter, a general scheme is presented to assess the feasibility of the $\mathrm{RNC}$ isolator its behavior under simultaneous horizontal and vertical loads in 
typical practical situations. Further, a mathematical description of the main features associated to rolling of the RNC isolator is presented. At the end, an input-output mathematical model is obtained to describe in a reasonable and manageable form the force-displacement relationship exhibited by the RNC isolator. 
This page intentionally left blank. 


\section{Isolation of heavy mass buildings}

\subsection{Introduction}

As effective isolation needs to achieve a long period response, most practical isolation systems work best with heavy masses. The period is proportional to the square root of mass $M$ and inversely proportional to the square root of the stiffness $K$ :

$$
T=2 \pi \sqrt{\frac{M}{K}}
$$

To achieve a given isolated period, a low mass must be associated with a low stiffness. The present day isolation devices do not have an infinite range of stiffness. Thus, heavy buildings isolation can achieve the required long period with no need to very low isolator stiffness.

The aim of this chapter is to perform an extensive numerical assessment of the RNC isolator performance considering this class of buildings. In order to do this, a heavy mass building structure supported by the RNC isolators is designed and simulated as a case study. Several designs of the RNC isolator are considered along with a wide range of structural fundamental periods and earthquake ground motions.

\subsection{The used RNC isolator type}

The RNC-a and RNC-b isolators, that are more suitable for heavy mass structures, are designed and used in this chapter. These RNC types are shown in Figs. 3.2 and 3.3, respectively. For the designed RNC-a isolator, the horizontal 
and vertical distances between the furthermost points are $3.177 \mathrm{~m}$ and $1.50 \mathrm{~m}$, respectively, whereas the horizontal and vertical distances between the furthermost points are $2.707 \mathrm{~m}$ and $1.50 \mathrm{~m}$, respectively, for the RNC- $\mathrm{b}$. The isolators dimensions are chosen to allow for $53 \mathrm{~cm}$ as a maximum rolling displacement, beyond which the buffer mechanism stops the isolated structure with minimal shock.

\subsection{Mechanical characteristics}

Following the mechanical characterization scheme described in Section 4.2, the designed RNC-a isolator used in this chapter has the following mechanical characteristics:

- Maximum vertical load capacity $=4000 \mathrm{kN}$.

- Pre-yield stiffness $=23508.6 \mathrm{kN} \mathrm{m}^{-1}$.

- Post-yield stiffness $=926.9 \mathrm{kN} \mathrm{m}^{-1}$.

- Yield displacement $=1.3 \mathrm{~cm}$.

- Yield force $=316.8 \mathrm{kN}$.

- Characteristic strength $=307.8 \mathrm{kN}$.

Similarly, the designed RNC-b isolator has the following mechanical characteristics:

- Maximum vertical load capacity $=4000 \mathrm{kN}$.

- Pre-yield stiffness $=95052.6 \mathrm{kN} \mathrm{m}^{-1}$.

- Post-yield stiffness $=8672.3 \mathrm{kN} \mathrm{m}^{-1}$.

- Yield displacement $=0.30 \mathrm{~cm}$.

- Yield force $=316.7 \mathrm{kN}$.

- Characteristic strength $=291.5 \mathrm{kN}$.

Further, and to draw relatively general conclusions about the performance of the RNC isolator under a variety of structural and ground motion properties, four different designs of each RNC isolator ranging from very stiff (sets I) to very flexible (sets IV) designs, are evaluated in this study. The maximum vertical load capacity per bearing is $4000 \mathrm{kN}$. Table 5.1 gives the basic mechanical characteristics of such designs. These characteristics have been also obtained following the scheme given in Section 4.2. 


\begin{tabular}{cccccc}
\hline $\begin{array}{c}\text { Isolator } \\
\text { Set }\end{array}$ & $\begin{array}{c}\text { Isolator } \\
\text { Period }\left(T_{\mathrm{b}}\right)\end{array}$ & $\begin{array}{c}\text { Elastic } \\
\text { Stiffness }\left(k_{\mathrm{e}}\right)\end{array}$ & $\begin{array}{c}\text { Post-yield } \\
\text { Stiffness }\left(k_{\mathrm{b}}\right)\end{array}$ & $\begin{array}{c}\text { Yield } \\
\text { Strength }\left(f_{\mathrm{y}}\right)\end{array}$ & $\begin{array}{c}\text { Characteristic } \\
\text { Strength }(Q)\end{array}$ \\
\hline \multicolumn{2}{c}{ RNC isolator type $\mathbf{a}$} & & & & \\
I & $4.04 \mathrm{sec}$ & 22261.6 & 877.8 & 300.0 & 291.6 \\
II & $5.70 \mathrm{sec}$ & 11130.8 & 438.9 & 150.0 & 145.8 \\
III & $8.05 \mathrm{sec}$ & 5565.4 & 219.5 & 75.0 & 72.9 \\
IV & $11.37 \mathrm{sec}$ & 2782.7 & 109.7 & 37.5 & 36.5 \\
\hline \multicolumn{2}{l}{ RNC isolator type $\boldsymbol{b}$} & & & & \\
I & $1.39 \mathrm{sec}$ & 90012.0 & 8212.4 & 300.0 & 276.0 \\
II & $1.91 \mathrm{sec}$ & 45006.0 & 4106.2 & 150.0 & 138.0 \\
III & $2.66 \mathrm{sec}$ & 22503.0 & 2053.1 & 75.0 & 69.0 \\
IV & $3.74 \mathrm{sec}$ & 11251.5 & 1026.6 & 37.5 & 34.5 \\
\hline
\end{tabular}

Table 5.1: Characteristics of different RNC isolator sets used in this study, kN-m units.

\subsection{Hysteretic modeling}

The characterization described in Chapter 4 has shown that the RNC isolator exhibits a hysteretic behavior, as shown in Fig. 4.7. The objective of this section is to obtain an input-output mathematical model to describe in a reasonable and manageable form the force-displacement relationship exhibited by the RNC-a and RNC-b isolators using the Bouc-Wen model of smooth hysteresis, which is described in Section 4.5. The standard Bouc-Wen model form is used in this chapter. Such form of the model is defined in Section 4.5.2.

\subsubsection{Bouc-Wen model parameters estimation}

By comparing the Bouc-Wen model output to the experimental (ANSYS) data, for a given periodic input displacement signal, the model parameters are estimated to characterize the RNC-a and RNC-b isolators. A total of 7 parameters $(A, \alpha, \beta, \gamma, D, n$, and $k$ ) are forced to obey the necessary conditions in Eq. (4.16).

A constrained nonlinear least-squares optimization algorithm, available in MATLAB [5], is used to obtain the 7 model parameters in Eqs. (4.14) and (4.14). The optimized parameters were determined to fit the model to the ANSYS output data. The resulting parameters are given in Table 5.2 for the $\mathrm{RNC}$ isolators types a and $\mathrm{b}$.

Fig. 5.1 shows the time history and the force-displacement relationship for the RNC-a isolator, considering both the steady-state actual response (ANSYS) and the steady-state predicted response (identified Bouc-Wen model). Fig. 5.1 clearly shows irregularities and randomness in the actual response. These 


\begin{tabular}{crllcc}
\hline Parameter & & Range & & Value $(\mathrm{RNC}-\mathrm{a})$ & Value $(\mathrm{RNC}-\mathrm{b})$ \\
\hline$A$ & 0 & $\leq A<$ & $\infty$ & 1.696 & 4.582 \\
$\alpha$ & 0 & $\leq \alpha \leq$ & 1 & 0.068 & 0.369 \\
$\beta$ & $\gamma$ & $\leq \beta<$ & $\infty$ & $8.212 \mathrm{e}-04$ & 0.022 \\
$\gamma$ & $-\beta$ & $\leq \gamma \leq$ & $\beta$ & $1.000 \mathrm{e}-05$ & $1.000 \mathrm{e}-05$ \\
$D$ & 0 & $\leq D<$ & $\infty$ & 0.004 & 0.001 \\
$n$ & 1 & $\leq n<$ & $\infty$ & 4.392 & 1.515 \\
$k$ & 0 & $\leq k<$ & $\infty$ & 602.98 & 1177.70 \\
\hline
\end{tabular}

Table 5.2: Identified parameters of the Bouc-Wen model to characterize the RNC-a and RNC-b isolators.

irregularities are expected to be due to the rolling of the stiff rolling body between upper and lower plates of the hyperelastic neoprene material. To check the validity of this assumption, the ANSYS model is modified in such a way that the horizontal force between the rolling body and the hyperelastic plates is canceled, while keeping the influence of the rolling body in the vertical direction. Under this condition, the experiment of Fig. 5.1 is repeated and the simplified ANSYS results are shown in Fig. 5.2 in thin-dashed line. For comparison purposes, Fig. 5.2 also plots the same Bouc-Wen model output as in Fig. 5.1, and the same bilinear approximation output as in Fig. 5.3. From Fig. 5.2, it can be seen that the resulting simplified ANSYS output is smooth. This means that the measured response irregularities in Fig. 5.1 can be attributed to the stiff rolling body contact with the hyperelastic neoprene material.

It is interesting to calculate the areas of the different hysteretic cycles plotted in Figs. 5.1 and 5.2 for comparison purposes. These areas are:

- Area of the actual ANSYS output $=10.34$ (average of three cycles)

- Area of the simplified ANSYS output $=10.14$

- Area of the Bouc-Wen model output $=10.38$

- Area of the Bilinear model output $=10.49$

This means that the simplified ANSYS, the Bouc-Wen and the bilinear outputs, all of them, are very close in shapes and areas as the actual ANSYS output. This implies that the mechanical characteristics obtained in Section 5.3 ( with the aid of bilinear approximation shown in Fig. 5.3) from the actual ANSYS output can be almost the same if obtained from the simplified ANSYS output shown in Fig. 5.2. Moreover, the energy dissipation per cycle by means of the Bouc-Wen model and the simplified ANSYS model are almost the same as that of the actual ANSYS model. Two main conclusions can be drawn: (1) The Bouc-Wen model is a good candidate for representing the energy dissipation mechanism of the RNC isolator; (2) The simplified ANSYS model is as accurate as the full ANSYS model in predicting the mechanical characteristics and the energy dissipation of the RNC isolator. 
$\mathbf{a}$

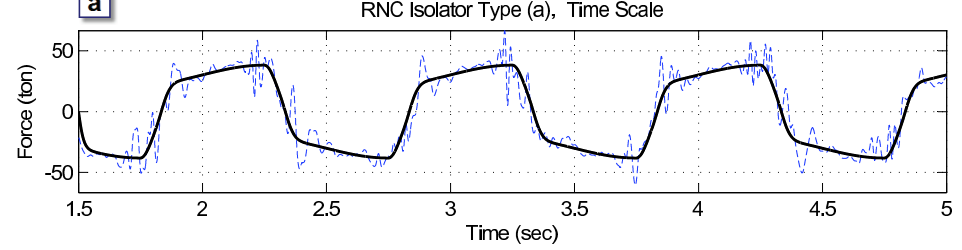

b

RNC Isolator Type (a), Displacement Scale

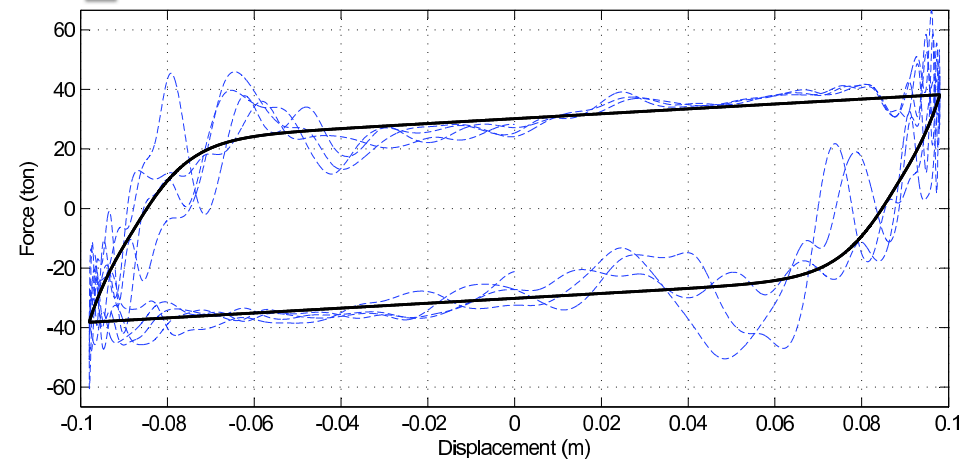

$---\cdot$ Actual ANSYS output, — Bouc-Wen model output

Figure 5.1: Actual ANSYS steady-state response versus predicted (Bouc-Wen model) steady-state response under harmonic input for the RNC-a isolator: (a) Time scale, (b) Displacement scale.

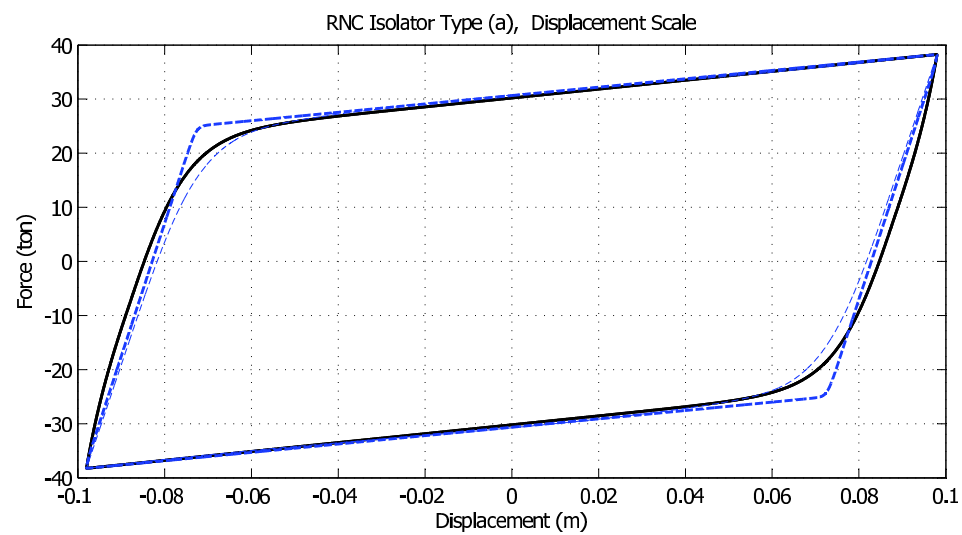

- Bouc-Wen model output, ----- Equivalent bilinear approximation, ----- Simplified ANSYS output.

Figure 5.2: Comparison between the simplified ANSYS output, the bilinear approximation and the identified Bouc-Wen model using the actual ANSYS output. 


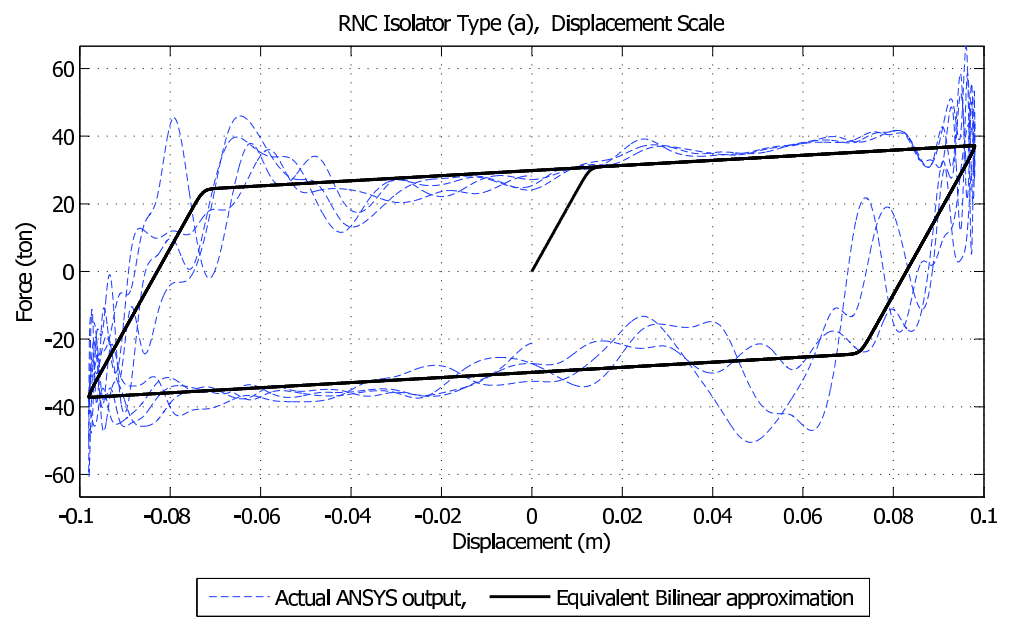

Figure 5.3: Actual ANSYS output force and its equivalent bilinear approximation, $\mathrm{RNC}-\mathrm{a}$ isolator.

Running the simulation of the full ANSYS model requires huge computational efforts and computer resources and long time for each run. The main benefit of the assumed simplification of the ANSYS model is to get the same mechanical characteristics of the RNC isolator and almost the same energy dissipation per cycle, but using much lower computer resources and incomparable short run time. As a result, more extensive case studies and design improvement of the RNC isolator can be performed with much less cost and reasonable accuracy. Therefore, the simplified ANSYS output is considered for the rest of this study and is referred to as the measured response.

\subsubsection{Seismic verification of the Bouc-Wen model}

To check the validity of the identified parameters, an actual random seismic displacement (Kern Country earthquake, Taft Lincoln school tunnel, July 21, 1952 ) is input into the ANSYS and the Bouc-Wen models. The input and the outputs are plotted in Fig. 5.4 for RNC-a and RNC-b isolators. The discrepancy between the measured and predicted outputs, $F_{\mathrm{m}}$ and $F_{\mathrm{b}}$, is quantified using the $L_{1}$ and $L_{\infty}$-norms and the corresponding relative errors $\varepsilon$ as expressed in (4.19).

As shown in Figs. 5.4(b,c) and with the small relative errors $\varepsilon_{1}$ and $\varepsilon_{\infty}$ in Table 5.3, the hysteretic Bouc-Wen model can be seen as a very powerful representation of the $\mathrm{RNC}$ isolator for further studies. 

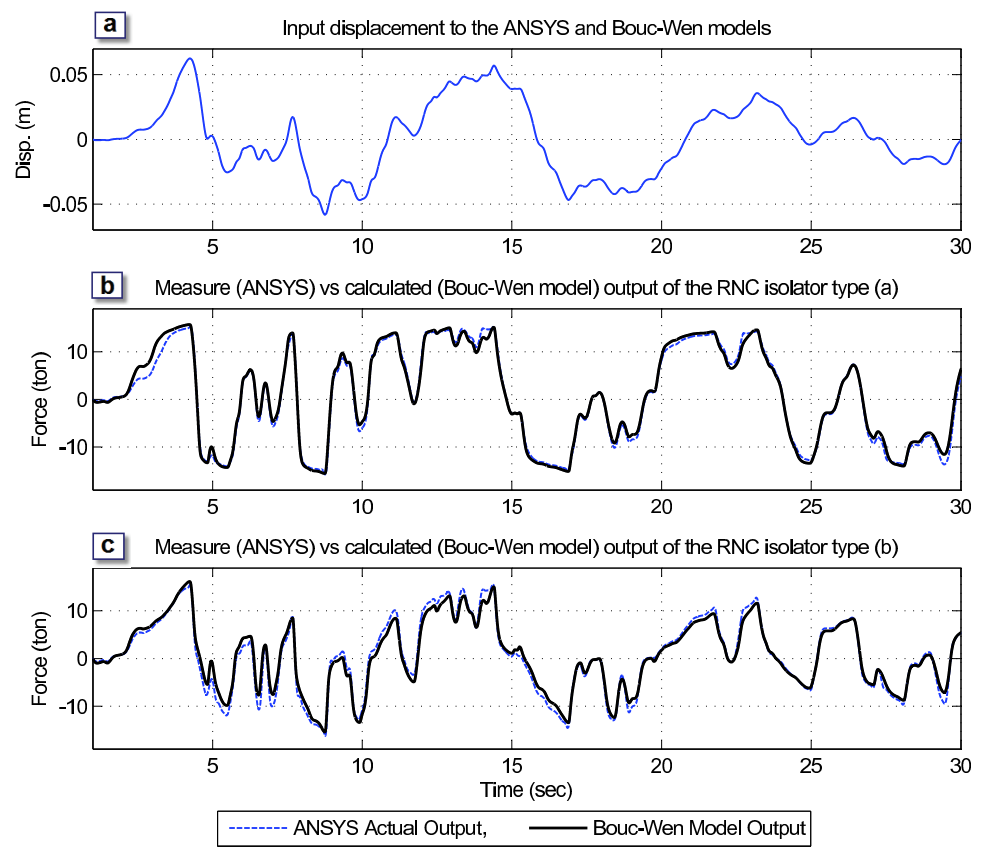

Figure 5.4: Bouc-Wen model validation: (a) Input seismic displacement, (b) Measured vs predicted restoring forces for RNC-a isolator, (c) Measured vs predicted restoring forces for RNC-b isolator.

\subsection{Implementation}

An idealized six bays, fifteen story (16-DOFs including the suspended base) base-isolated concrete moment-resisting frame is considered in the present study as a case-study structure. It is modeled as a shear type structure mounted on the proposed isolation bearing, Fig. 5.5, with one lateral degree-of-freedom DOF at each floor where all the vibrational modes are included in the analysis. The following assumptions are made for the structural system under consideration:

1. The superstructure remains within the elastic limit during the earthquake excitation.

2. The floors are assumed rigid in its own plane and the mass is lumped at each floor level.

3. The columns are inextensible and weightless providing the lateral stiffness.

4. The system is subjected to single horizontal component of the earthquake ground motion. 


\begin{tabular}{ccc}
\hline Discrepancy & \multicolumn{2}{c}{ Bouc-Wen model } \\
\cline { 2 - 3 } Measure & RNC type a & RNC type b \\
\hline$\varepsilon_{1}$ & 0.108 & 3.616 \\
$\varepsilon_{\infty}$ & 0.205 & 0.632 \\
\hline
\end{tabular}

Table 5.3: Discrepancy between measured and predicted outputs under seismic input, for both RNC-a and RNC-b isolators.

5. The effects of soil-structure interaction are not taken into consideration.

The cross sectional dimensions of the frame columns and beams are $1.2 \times$ $0.30 \mathrm{~m}$ and $0.80 \times 0.30 \mathrm{~m}$, respectively. All the stories are $3.0 \mathrm{~m}$ height and the frame span is $6.0 \mathrm{~m}$. The frame material is normal-weight concrete with a total material volume per frame of $255 \mathrm{~m}^{3}$. This concrete material has the following isotropic properties:

- Weight per unit volume $=23563.12 \mathrm{~N} \mathrm{~m}^{-3}$

- Mass per unit volume $=2402.77 \mathrm{Kg} \mathrm{m}^{-3}$

- Modulus of elasticity $=2.486 \mathrm{E}+10 \mathrm{~N} \mathrm{~m}^{-2}$

- Poisson's ratio $=0.20$

- Shear modulus $=1.036 \mathrm{E}+10 \mathrm{~N} \mathrm{~m}^{-2}$

- Specified concrete compressive strength $=27579032 \mathrm{~N} \mathrm{~m}^{-2}$

The superstructure is idealized as a linear flexible building. The modal frequencies, periods, and the modal mass participation factor of the designed structure are listed in Table 5.4 where the damping ratio for all modes is kept fixed to $2 \%$ of the critical damping. All vibration modes are considered, while only the first relevant modes are listed in Table 5.4. The total weight of the example frame structure (including the isolation bearings) is $25000 \mathrm{kN}$ supported by $7 \mathrm{RNC}$ isolators, one under each column.

\subsubsection{Equations of motion}

The equations of motion of the $N$-story linear shear type superstructure subjected to earthquake excitation is written in the matrix form

$$
\mathbf{M}_{\mathrm{s}} \ddot{\mathbf{x}}_{\mathrm{s}}+\mathbf{C}_{\mathrm{s}} \dot{\mathbf{x}}_{\mathrm{s}}+\mathbf{K}_{\mathrm{s}} \mathbf{x}_{\mathrm{s}}=-\mathbf{M}_{\mathrm{s}}\{\mathbf{1}\}\left(\ddot{x}_{\mathrm{b}}+\ddot{x}_{\mathrm{g}}\right),
$$

where $\mathbf{M}_{\mathrm{s}}, \mathbf{K}_{\mathrm{s}}$, and $\mathbf{C}_{\mathrm{s}}$ are the $N \times N$ mass, stiffness and damping matrices of the superstructure, respectively; $\mathbf{x}_{\mathrm{s}}=\left\{x_{1}, x_{2}, \ldots, x_{N}\right\}^{T}$ is the relative displacement vector of the superstructure; $\dot{\mathbf{x}}_{\mathrm{s}}$ and $\ddot{\mathbf{x}}_{\mathrm{S}}$ are the relative velocity and 


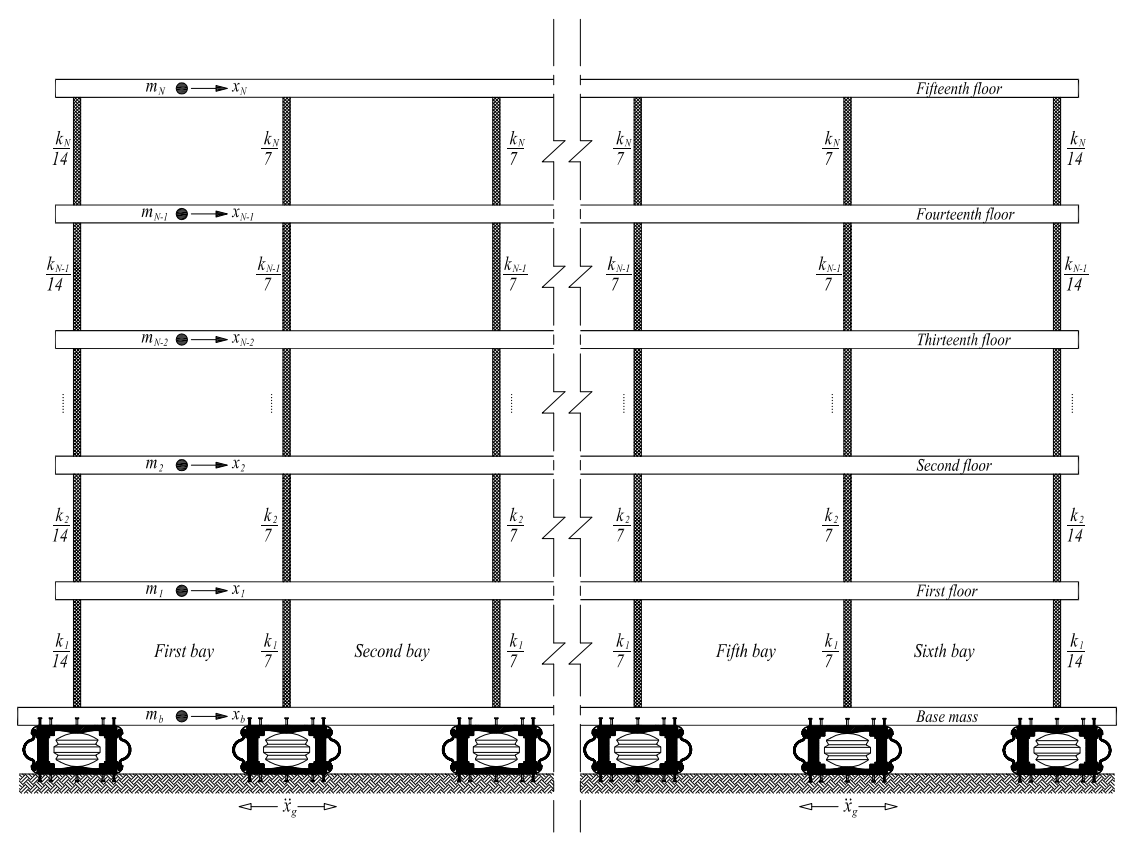

Figure 5.5: Case-study structure isolated by the RNC isolator

acceleration vectors, respectively; $x_{j}(j=1,2, \ldots, N)$ is the lateral displacement of the $j$ th floor relative to the base mass; $\{\mathbf{1}\}=\{1,1,1, \ldots, 1\}^{T}$ is the influence coefficient vector; $\ddot{x}_{\mathrm{b}}$ is the relative acceleration of the base mass; and $\ddot{x}_{\mathrm{g}}$ is the earthquake ground acceleration.

The governing equation of motion for the base mass is given by

$$
m_{\mathrm{b}} \ddot{x}_{\mathrm{b}}+\eta F_{\mathrm{b}}-c_{1} \dot{x}_{1}-k_{1} x_{1}=-m_{\mathrm{b}} \ddot{x}_{\mathrm{g}},
$$

where $m_{\mathrm{b}}$ is the mass of the base raft; $c_{1}$ and $k_{1}$ are the damping and stiffness of the first story, respectively; $\eta$ is the total number of isolators and $F_{\mathrm{b}}$ is the restoring force transmitted to the base mass by a single RNC isolator. This force is expressed by the Bouc-Wen model as

$$
\begin{aligned}
F_{\mathrm{b}}(t) & =\alpha k x_{\mathrm{b}}(t)+(1-\alpha) D_{\mathrm{y}} k z(t), \\
\dot{z} & =D_{\mathrm{y}}^{-1}\left(A \dot{x}_{\mathrm{b}}-\beta\left|\dot{x}_{\mathrm{b}}\right||z|^{n-1} z-\gamma \dot{x}_{\mathrm{b}}|z|^{n}\right),
\end{aligned}
$$

where $x_{\mathrm{b}}$ is the relative base displacement, $\alpha k x$ is the elastic force component, $z$ is an auxiliary variable, $\dot{z}$ denotes the time derivative, $n>1$ is a parameter governs the smoothness of the transition from elastic to plastic response, $D_{\mathrm{y}}>0$ is the yield constant displacement, $k>0$ and $0<\alpha<1$ represents the post to pre-yielding stiffness ratio $\left(k_{\mathrm{b}} / k_{\mathrm{e}}\right)$, while $A, \beta$ and $\gamma$ are non-dimensional parameters that govern the shape and size of the hysteresis loop. 


\subsubsection{Performance measures}

The response quantities of interest are the top floor absolute acceleration, building drift, base shear and the relative-to-ground base displacement. These response quantities are of importance because floor accelerations developed in the superstructure are a measure of human comfort and are the main source of damaging housed sensitive equipment. The building drift is the main cause of structural and nonstructural damage. The base shear and base moment govern the cross sectional dimensions of the lateral force supporting systems in structures, while the bearing (base) displacements are crucial in the design of isolation systems.

\subsubsection{Simulation tool}

Having the input data and the assumptions given in Section 5.5, the case study structure shown in Fig. 5.5 is modeled using the Structural Analysis Program SAP2000 advanced [6]. The proposed RNC isolator is modeled as a nonlinear support, whose dynamic behavior is governed by the hysteretic Bouc-Wen model, (5.4)-(5.5), where the rest of the structure is assumed to behave linearly. The parameters of the Bouc-Wen model have been identified, in Section 5.4.1 and the mechanical characteristics of the RNC-a and RNC$\mathrm{b}$ isolators are obtained in Section 5.3 and incorporated into the simulation code along with the isolator mass. Then the structural mass, stiffness and damping matrices are formed and related to other variables as shown explicitly by Eqs. (5.2)-(5.3). A modal analysis is performed first to determine the dynamic properties, of the modeled system, that are listed in Table 5.4. Then, a linear and nonlinear dynamic analysis are carried out in cases of fixed-base and isolated base structures, respectively, under a variety of ground motion excitations to determine the response quantities given in Section 5.5.2.

The dynamic response is calculated in SAP2000 using the fast nonlinear analysis FNA [462], which is more suitable for structures having limited number of points or members in which nonlinear behavior takes place when subjected to static or dynamic loading. This FNA makes the nonlinear analysis almost as fast as a linear analysis keeping the accuracy of accurate direct integration methods. The FNA method is applied to both the static and dynamic analysis of linear or nonlinear structural systems. A limited number of predefined nonlinear elements are assumed to exist. Stiffness and mass orthogonal Load Dependent Ritz Vectors of the elastic structural system are used to reduce the size of the nonlinear system to be solved. The forces in the nonlinear elements are calculated by iteration at the end of each time or load step. The uncoupled modal equations are solved exactly for each time increment. 


\subsubsection{Numerical study}

In the following numerical study, thorough investigation of the RNC isolator has been carried out to assess its effectiveness considering two main possible designs RNC-a and RNC-b. In Section 5.6, the behavior of the RNC isolator is investigated under harmonic ground motions, using frequency response analysis, considering different RNC characteristics. Section 5.7 briefly investigates some characteristics of the RNC isolator, and presents a comparison between fixed-base and base-isolated structural responses using time history analysis. The influence of the superstructural flexibility is studied in Section 5.8. The effect of long period earthquakes is considered in Section 5.9. Finally, the influence of different earthquake characteristics is examined in Section 5.10.

\subsection{Effect of isolator characteristics}

The four different designs of each RNC isolator types a and b are studied under harmonic ground motion, which is defined as a sinusoidal ground acceleration

$$
\ddot{x}_{\mathrm{g}}(t)=0.5 g \sin \left(2 \pi f_{\mathrm{g}} t\right)
$$

where $f_{\mathrm{g}}$ denotes the excitation frequency and $g$ is the gravitational acceleration.

Fig. 5.6(a) shows the peak structural absolute acceleration as a function of the excitation frequency in $\mathrm{Hz}$, using the four RNC-a isolator designs. The structural acceleration in the fixed-base structure is included for comparison. Fig. 5.6(b) shows the corresponding peak base floor displacement. By comparing the frequency response curves in Fig. 5.6(a), one observes that except for very low excitation frequencies, which are unlikely to occur, the structural absolute acceleration is significantly reduced. This response reduction grows as we move from the very stiff design ( $\mathrm{RNC}-$ a set $\mathrm{I}$ ) toward the very flexible one (RNC-a set IV). There is a single resonant peak at the structural fundamental frequency $(1.99 \mathrm{~Hz})$ in the fixed-base case. For isolator sets I and II, two resonant peaks are clear at low frequencies of $0.25 \mathrm{~Hz}$ and $0.18 \mathrm{~Hz}$, respectively. Moreover, some secondary peaks occur around the resonant frequency of the fixed-base case. These secondary peaks become clearer as the isolator flexibility increases (sets III and IV). The corresponding base displacement shown in Fig. 5.6(b) seem to be within reasonable ranges, except those at very low excitation frequencies. These base displacements become larger as the isolator flexibility increases. Further, resonant peaks occur for the stiffer isolator sets I and II.

Fig. 5.7(a) shows the peak structural accelerations against the excitation frequency in $\mathrm{Hz}$, using the four $\mathrm{RNC}-\mathrm{b}$ isolator designs. The main differences in behavior, relative to that of the $\mathrm{RNC}-\mathrm{a}$, arise from the relatively stiff nature of the RNC-b isolators. Such nature limits the isolator efficiency, as can 

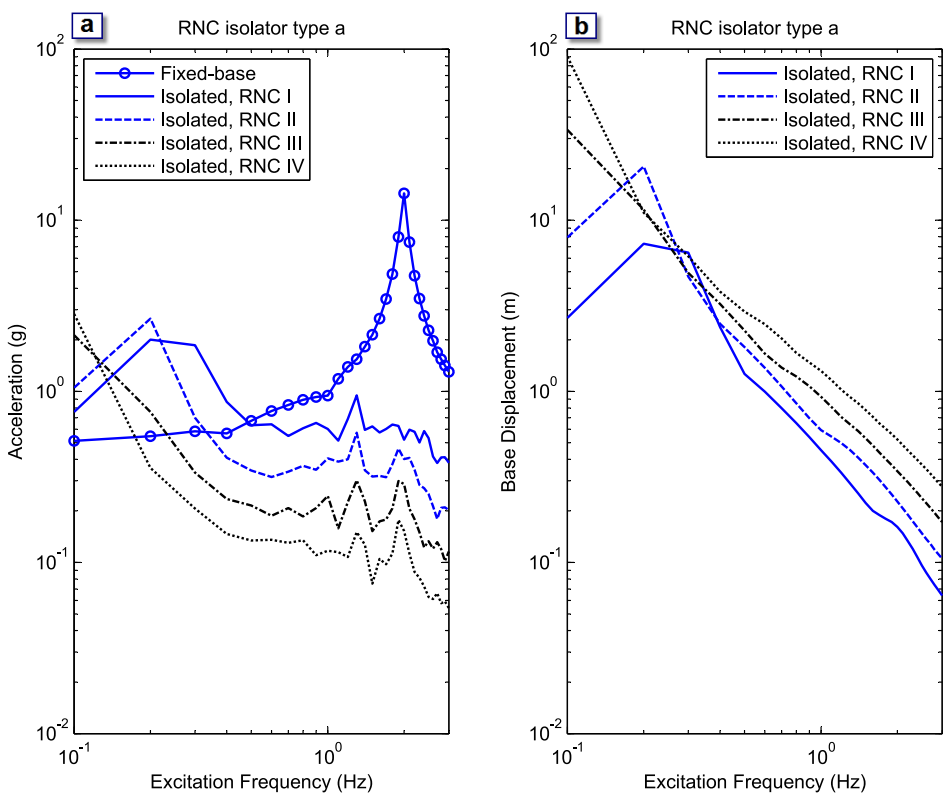

Figure 5.6: Frequency response of different designs of RNC-a isolator: (a) Absolute acceleration at the topmost point, (b) Relative-to-ground base displacement.

be seen when we compare Figs 5.6(a) and 5.7(a), where the isolated acceleration response is lower using $\mathrm{RNC}-\mathrm{a}$, around the fixed-base resonant frequency. Moreover, this higher stiffness shifts the resonant peaks in the base-isolated cases toward higher frequencies, which increases the probability of their occurrence. These resonant peaks are $0.72 \mathrm{~Hz}, 0.52 \mathrm{~Hz}, 0.38 \mathrm{~Hz}$, and $0.27 \mathrm{~Hz}$ for isolator sets I, II, III, and IV, respectively. In Fig. 5.7(b), the corresponding base displacements are plotted versus ground motion frequency. In this figure, four resonant peaks are evident when compared to Fig. 5.6(b). However, in Fig. 5.7(b) the frequency response curves has steeper slopes from the peaks on. This means that, due to the relatively higher stiffness of the RNC-b isolator sets, they exhibit higher rate of displacement reduction, specially as we move from the resonant peaks toward higher frequencies.

As can be seen, the acceleration response reduction is maximum in the cases of very flexible designs (sets IV) for RNC-a and RNC-b. However, the associated base displacements are the highest, but are still affordable. Moreover, the efficiency of the RNC-a is significantly higher than the RNC-b in reducing accelerations due to its higher flexibility. Further, the probability of resonance using the $\mathrm{RNC}-\mathrm{a}$ is far from occurrence if compared to the $\mathrm{RNC}-\mathrm{b}$, that might be more likely to occur.

Practically, seismic isolators have to resist minor vibrations. Lower pre- 

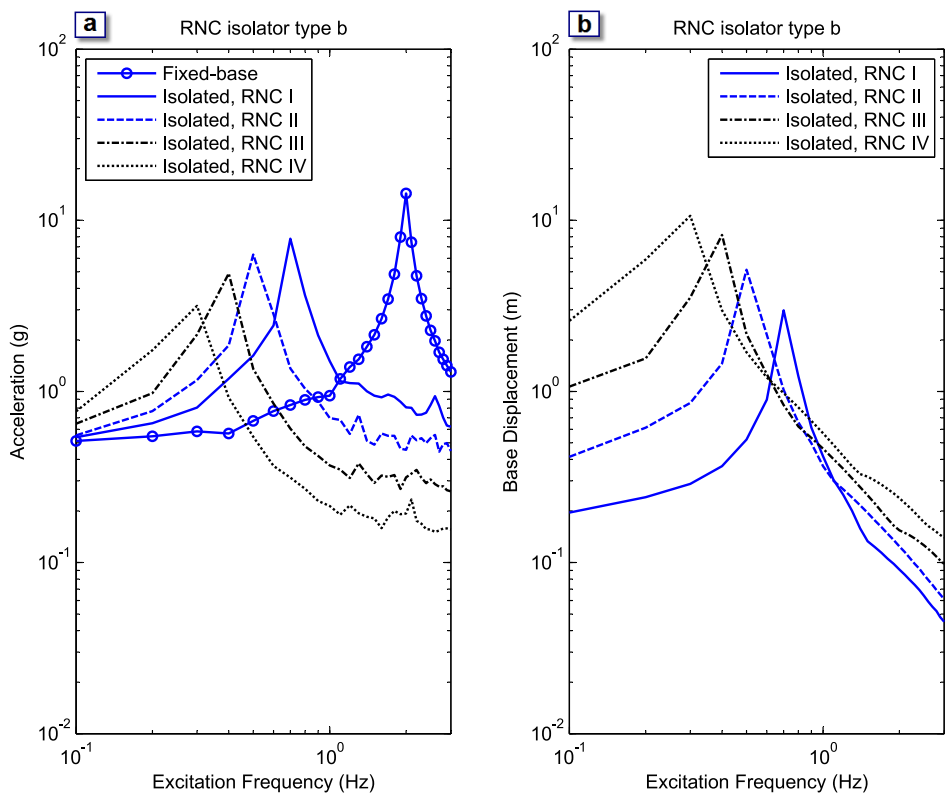

Figure 5.7: Frequency response of different designs of RNC-b isolator: (a) Absolute acceleration at the topmost point, (b) Relative-to-ground base displacement

yield stiffness renders the isolator highly sensitive to minor vibrations. Considering that the wind loads represent the source of minor vibrations, the structure in Fig. 5.5 has been subjected to a wind pressure of $2 \mathrm{kN} \mathrm{m}^{-2}$ including suction. Although the details are omitted here, it has been found that the RNC isolator sets II for RNC-a and RNC-b (see Table 5.1) offer adequate initial stiffness to resist the presumed wind pressure on the structure. Sets I are also practical with respect to wind resistance, but their higher stiffness render them less efficient for seismic response attenuation. On the other hand, design sets III and IV are softer than sets II, and therefore are impractical with respect to wind resistance. Therefore, only the RNC design sets II are considered in the rest of this study.

\subsection{Recentering, damping and time response}

In this section, the inherent gravity-based recentering mechanism of the RNC isolator is verified and the damping added to the structure by the RNC isolator is estimated. Moreover, a preliminary impression about the efficiency of the $\mathrm{RNC}$ isolator is briefly sought under earthquake ground motion.

The structure shown in Fig. 5.5, isolated by the RNC-b isolator set II, 
is subjected to a harmonic base excitation of $0.40 \mathrm{~Hz}$ for $50 \mathrm{sec}$, and then the amplitude of the excitation is set to zero to allow the system to go through a free vibration. Fig. 5.8(a) displays the absolute structural acceleration time history at a topmost point. It is clear that the system reaches the steady state within the first few cycles, and the influence of higher modal frequencies is small. Fig. 5.8(b) displays the base displacement time history. It can be seen that there is no residual displacement. Certainly, this highlights the efficiency of the gravity-based restoring mechanism, which is an inherent characteristic of the proposed RNC isolator.
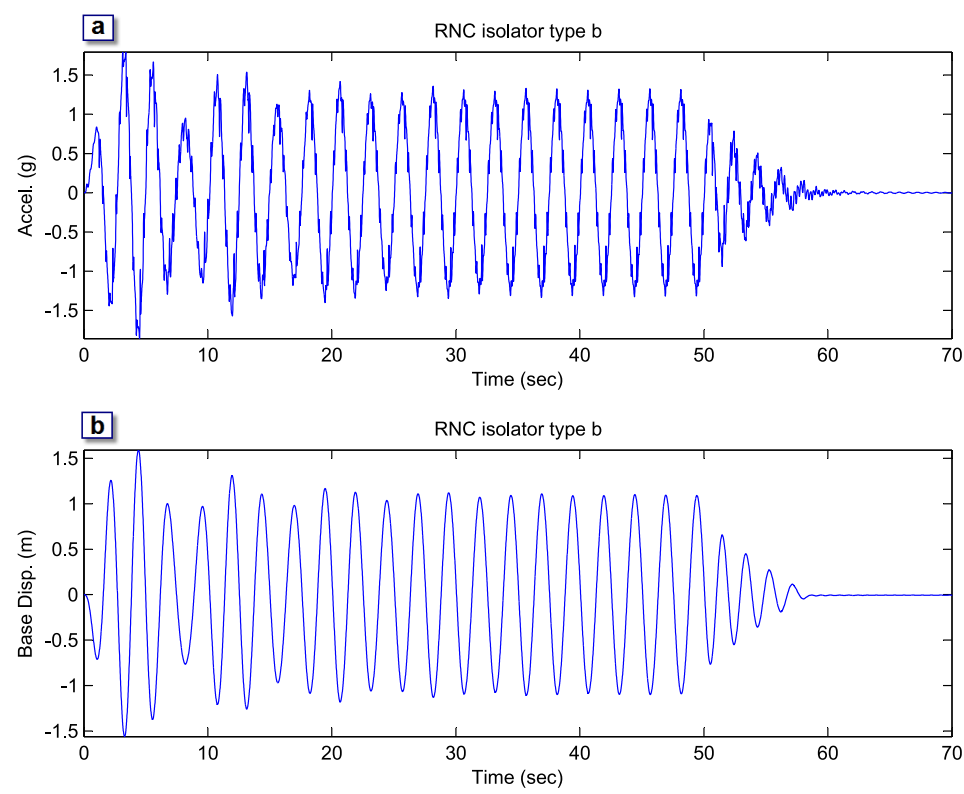

Figure 5.8: Harmonic response time history, RNC type b, set II: (a) Absolute acceleration at the topmost point, (b) Relative displacement at the base mass

The base motion decay during the free vibration mode in Fig. 5.8(b) represents a useful tool for estimating the damping provided by the $\mathrm{RNC}-\mathrm{b}$ isolator. The relation between the ratio of two consecutive peaks displacements $x_{\mathrm{i}}$ and $x_{\mathrm{i}+1}$ of damped free vibration and the damping ratio $\xi$ is [99]:

$$
\ln \frac{x_{\mathrm{i}}}{x_{\mathrm{i}+1}}=\frac{2 \pi \xi}{\sqrt{1-\xi^{2}}}
$$

As a result, the damping ratio $\xi$ using the RNC-b is $9 \%$. Similarly, the damping ratio $\xi$ using the $\mathrm{RNC}-\mathrm{a}$ is $13 \%$.

The time history of the system response under the strong Kobe earthquake excitation is presented in Figs. 5.9 and 5.10. Figs. 5.9(a,b) show the fixedbase and base-isolated absolute acceleration of the topmost floor using RNC-a 
and RNC-b, respectively. The figures show the ability of the RNC isolator to reduce the structural responses. This reduction is more significant using the RNC-a. This is mainly due to the lower post-yield stiffness in the case of $\mathrm{RNC}-\mathrm{a}$, as it was already pointed out in Section 5.6.
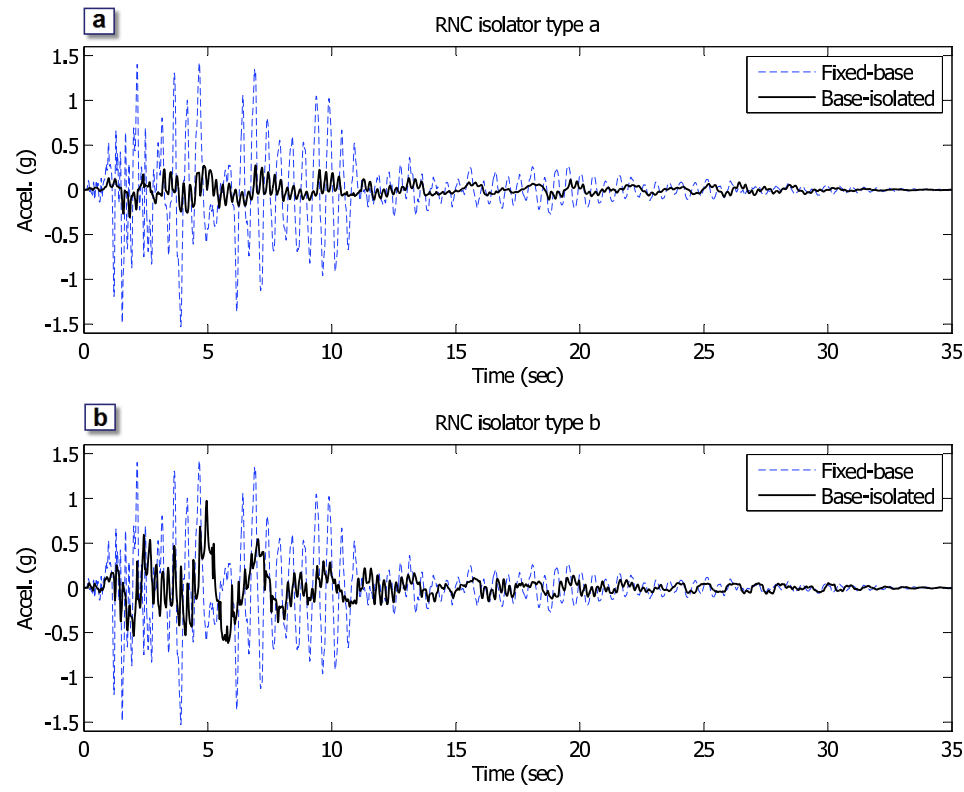

Figure 5.9: Time history of top floor acceleration response under Kobe earthquake, using sets II: (a) Fixed-base vs base-isolated structure by RNC-a, (b) Fixed-base vs baseisolated structure by RNC-b.

The peak accelerations at the top floor of the base-isolated structure are 0.307 and $0.973 \mathrm{~g}$ for the $\mathrm{RNC}-\mathrm{a}$ and $\mathrm{RNC}-\mathrm{b}$, respectively, while the peak acceleration at the same point of the fixed-base structure is $1.529 \mathrm{~g}$.

In a similar manner, the corresponding relative base displacements under the same excitation are plotted in Fig. 5.10. Figs. 5.10(a,b) show the relative bearing displacement using $\mathrm{RNC}-\mathrm{a}$ and $\mathrm{RNC}-\mathrm{b}$, respectively. The peak bearing displacements for the base-isolated structure are 0.349 and $0.514 \mathrm{~m}$ for the RNC-a and RNC-b, respectively. This indicates that the bearing displacements can be reasonable and affordable, especially for RNC-a, under such strong motion Kobe earthquake.

\subsection{Influence of superstructure flexibility}

The isolation systems are robust and practically useful when they are effective for different structures with wide range of properties. The fundamental time 

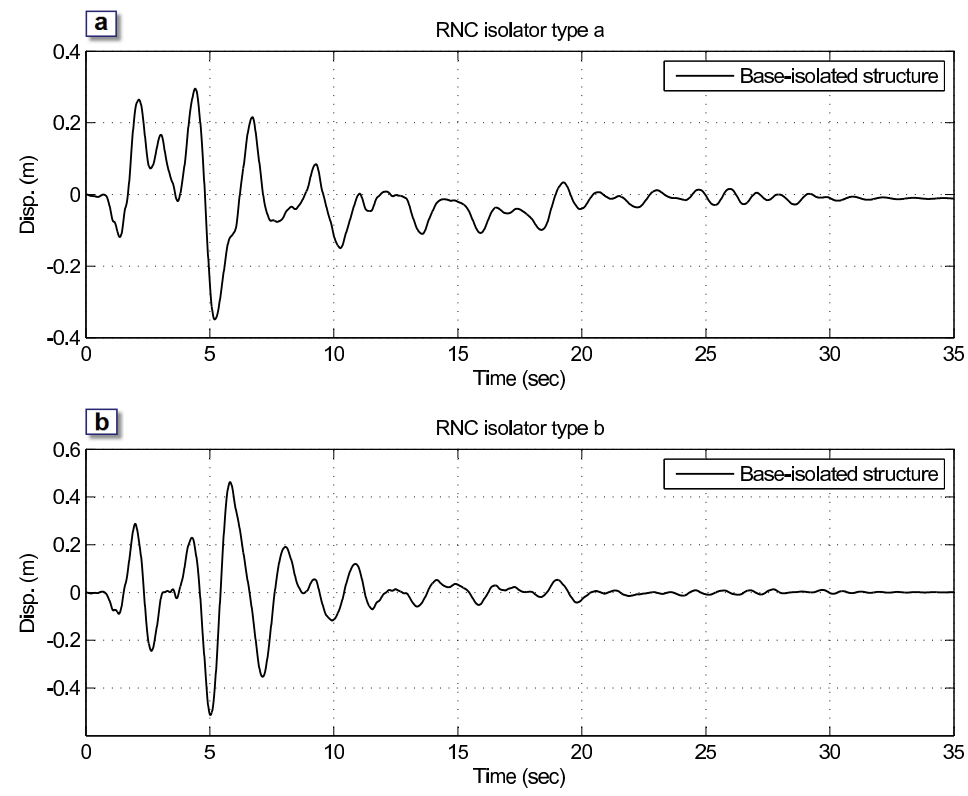

Figure 5.10: Time history of bearing relative displacement response under Kobe earthquake, using sets II: (a) Base-isolated structures by RNC-a, (b) Base-isolated structures by RNC-b.

period of a structure is the most important property from the dynamic response point of view. For most typical building structures, the fundamental time period varies between 0.1 to $0.5 \mathrm{~s}$. Tall buildings and flexible structures have periods of up to $2 \mathrm{~s}$ or longer. A base isolator derives its effectiveness by increasing the time period of the structure. It is therefore essential that this period be shorter than the time period of the isolator. In this investigation, two RNC isolator sets II (one for each type a and b) are considered to isolate case-study structures with different fundamental periods within a wide range from 0.1 to $2.0 \mathrm{sec}$. This choice corresponds to the most likely practical values of these parameters.

The response spectra for the structural absolute acceleration, at the topmost point, and the relative base displacement are shown in Fig. 5.11 for three earthquakes of different intensities, ranging from low to severe peak ground accelerations (PGA). The three earthquakes are Kern (low intensity, PGA = $0.18 g$ ), El-Centro (moderate intensity, PGA $=0.35 g$ ) and San-Fernando (severe intensity, $\mathrm{PGA}=1.17 \mathrm{~g}$ ) earthquakes. The following conclusions can be drawn drawn after analyzing the results in Fig. 5.11:

- The isolation efficiency increases as the earthquake intensity increases.

- The RNC-a isolator offers better isolation performance than the RNC-b 

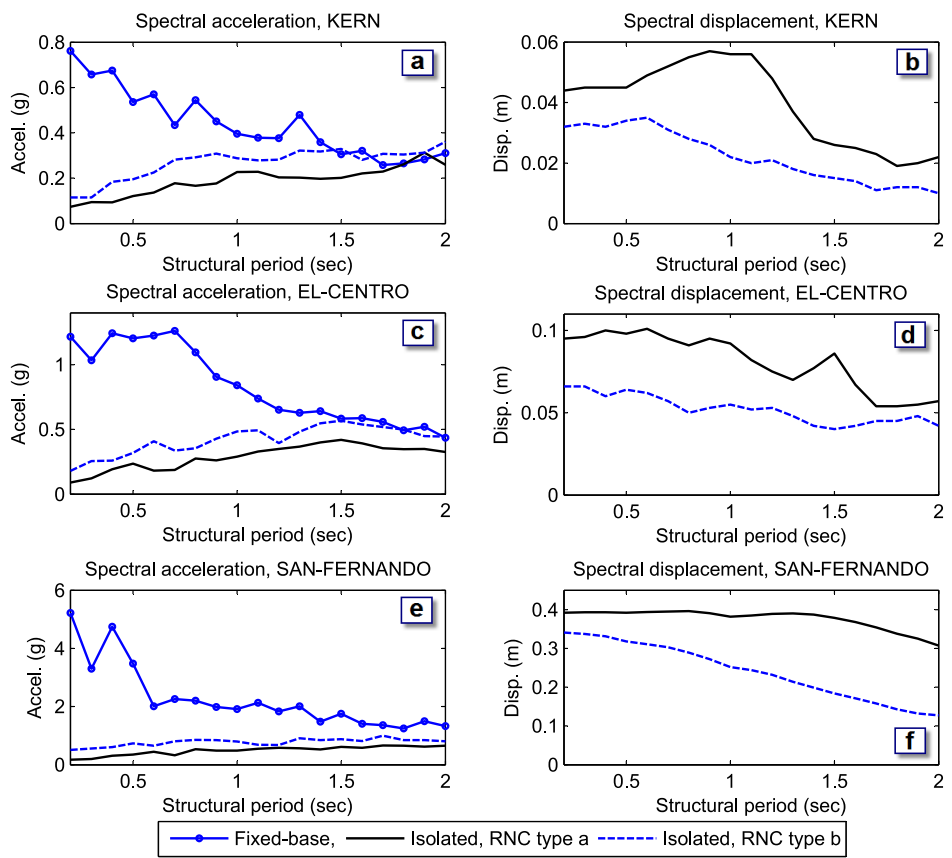

Figure 5.11: Acceleration and displacement response spectra under three earthquakes: (a) Acceleration of the top floor under Kern earthquake, (b) Base displacement under Kern earthquake, (c) Acceleration of the top floor under El Centro earthquake, (d) Base displacement under El Centro earthquake, (e) Acceleration of the top floor under San Fernando earthquake, (f) Base displacement under San Fernando earthquake.

isolator, but it is accompanied with larger base displacement, which is still within reasonable ranges.

- Although the isolation efficiency decreases as the structural fundamental period increases, the RNC isolator is still valid for very long fundamental periods, specially under severe intensity earthquakes.

- Under all intensities, the maximum acceleration is almost independent of the structure time period, particularly under the highest earthquake intensity (San Fernando).

Therefore, the RNC isolator is a robust isolation device, as it exhibits a relatively similar behavior for a very wide range of structural time periods, under the same earthquake intensity. 


\subsection{Behavior under long-period earthquakes}

This section investigates the usefulness of the proposed RNC isolator under long-period seismic excitations. The Mexico City 1985 earthquake has been used as an excitation for fixed-base and isolated-base cases. The whole building drift (difference between the topmost and base displacements), absolute acceleration of top floor, and base shear are chosen as performance measures, and they are plotted against the structural fundamental period in Fig. 5.12. From this figure, the following notes are drawn:

- The RNC-a isolator reduces the building drift significantly for all structural periods, specially at long structural periods, contrary to RNC-b.

- The RNC-a is reasonably effective in reducing the acceleration response at short periods (up to $0.60 \mathrm{sec}$ ), whereas it offers a relatively neutral behavior for higher periods.

- Considering the base shear, the RNC-a exhibits a robust behavior for all the structural periods considered.

- For all the performance measures, the RNC-b isolator appears to be ineffective or at maximum neutral for periods up to almost $1.60 \mathrm{sec}$, but it shows some efficiency at longer periods. This emphasizes that the $\mathrm{RNC}-\mathrm{a}$ is much superior to the RNC-b isolator under such long-period earthquake.

\subsection{Influence of earthquake characteristics}

Robust performance of the RNC isolator against changes of earthquake characteristics is investigated in this Section. This investigation is carried out following three steps:

1. In the first step, the RNC-a is considered as the isolation device in the case-study structure of Fig. 5.5 under 3 scaled intensities (PGA $=50 \%$, $100 \%$ and $200 \%$ ) of El Centro earthquake, in order to investigate the influence of earthquake amplitude on the RNC behavior. The results of this step are listed in Table 5.5.

2. In the second step, the RNC-a is used along with three other earthquakes (Kobe, Northridge and Parkfield) of equally scaled amplitudes to $0.5 \mathrm{~g}$ as the excitation source. In this step, a relation between the RNC-a isolator performance and the frequency content is sought. The results of this step are listed in Table 5.6. In both first and second steps, a wide range of structural fundamental periods $\left(T_{\mathrm{s}}=0.20-2.0 \mathrm{sec}\right)$ is considered. 

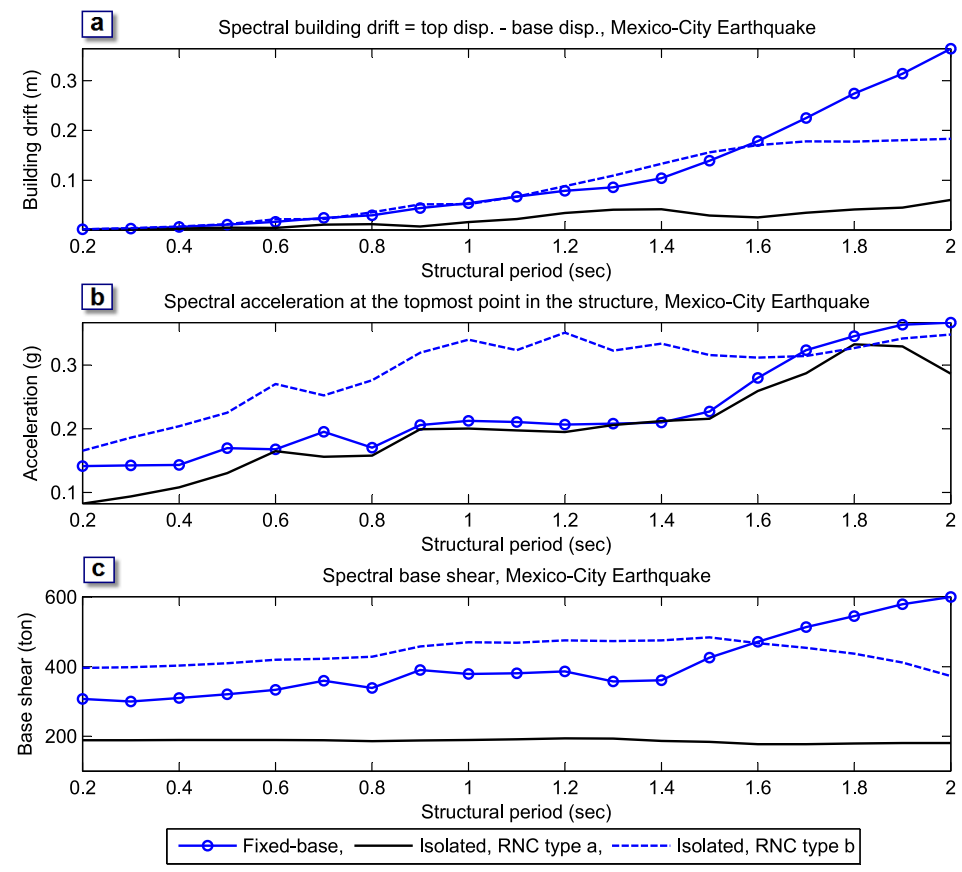

Figure 5.12: Response spectra under long-period Mexico City earthquake: (a) Building drift, (b) Acceleration at top floor, (c) Base shear.

3. Finally, and to confirm the results obtained from the first two steps, a set of 36 earthquakes is used to excite the structure shown in Fig. 5.5 with a fundamental period of $0.50 \mathrm{sec}$. In this step, both RNC types a and b are examined, and the results are listed in Table 5.7.

From Table 5.5, it can be observed that the fixed base responses are doubled as the excitation amplitudes are doubled, which is not always the case for the RNC-isolated structural acceleration response. In all cases, the attenuation of the structural absolute acceleration (at top floor) by means of the RNC-a isolator is evident, especially under higher intensities and short structural periods. This response attenuation is accompanied with affordable base displacements, under all conditions. This may clearly highlight the independence of the RNC isolator efficiency on the excitation amplitude.

Fig. 5.13 shows the normalized Fourier amplitude of the three used earthquakes in the second step. Comparing the structural response in Table 5.6 and 

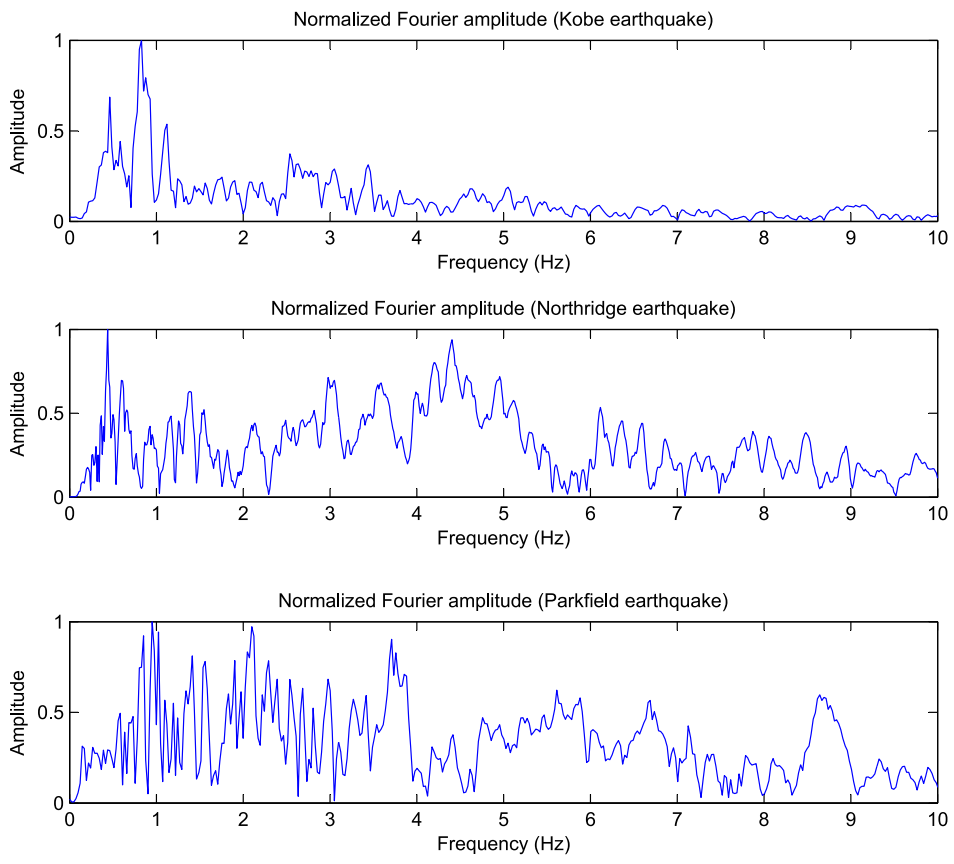

Figure 5.13: Normalized Fourier amplitude of: (a) Kobe earthquake, (b) Northridge earthquake, (c)Parkfield earthquake.

the Fourier amplitudes in Fig. 5.13 for each earthquake, it is obvious that there is no clear relation between the RNC-a isolator efficiency and the frequency content. Moreover, significant acceleration response attenuation is apparent with reasonable base displacement.

Table 5.7 presents the results of the extensive study under a wide set of distinct earthquake ground motions (third step). The building drift, the top floor absolute acceleration and the base shear are the three used performance measures. From this table, the following main observations can be emphasized:

- The RNC-a always mitigates very substantially the building drift, the absolute acceleration at the topmost floor and the base shear, on the account of reasonable bearing displacement.

- Except for Mexico City earthquake, the RNC-b is also efficient in mitigating the same seismic responses, although it is not as effective as the RNC-a.

In summary, the RNC isolator may be seen as a robust isolation device under variations in earthquake characteristics. 


\subsection{Conclusions}

Chapters 3 and 4 presented a detailed description of the main principles of operation, modeling and characterization of the RNC-a and RNC-b isolators. In this chapter, an extensive numerical assessment of the performance of such RNC isolators has been presented. The numerical investigations affirmed the effectiveness of the device by attaining significant reduction of the building accelerations, drifts and base shears, while keeping reasonable base displacement. Even when very flexible structures (time period exceeding $1.0 \mathrm{sec}$ ) are isolated and long time-period excitations are considered, the proposed system is found to be highly effective. Further, the proposed RNC isolation bearing is found to exhibit a robust performance for a wide range of structures, isolators and ground motion characteristics. 


\begin{tabular}{|c|c|c|c|c|c|c|c|c|c|}
\hline \multirow{3}{*}{$T_{\mathrm{s}}$} & \multicolumn{3}{|c|}{ El-Centro $50 \%$} & \multicolumn{3}{|c|}{ El-Centro $100 \%$} & \multicolumn{3}{|c|}{ El-Centro $200 \%$} \\
\hline & \multirow{2}{*}{$\begin{array}{r}\text { Fixed } \\
\text { Top } \\
\text { Accel. }\end{array}$} & \multicolumn{2}{|c|}{ Isolated } & \multirow{2}{*}{$\begin{array}{r}\text { Fixed } \\
\text { Top } \\
\text { Accel. }\end{array}$} & \multicolumn{2}{|c|}{ Isolated } & \multirow{2}{*}{$\begin{array}{r}\text { Fixed } \\
\text { Top } \\
\text { Accel. }\end{array}$} & \multicolumn{2}{|c|}{ Isolated } \\
\hline & & $\begin{array}{l}\text { Base } \\
\text { Disp. }\end{array}$ & $\begin{array}{r}\text { Top } \\
\text { Accel. }\end{array}$ & & $\begin{array}{l}\text { Base } \\
\text { Disp. }\end{array}$ & $\begin{array}{r}\text { Top } \\
\text { Accel. }\end{array}$ & & $\begin{array}{l}\text { Base } \\
\text { Disp. }\end{array}$ & $\begin{array}{r}\text { Top } \\
\text { Accel. }\end{array}$ \\
\hline 0.20 & 5.944 & 0.066 & 0.533 & 11.888 & 0.138 & 0.659 & 23.777 & 0.230 & 1.209 \\
\hline 0.30 & 5.412 & 0.066 & 0.643 & 10.824 & 0.139 & 0.919 & 21.649 & 0.231 & 1.214 \\
\hline 0.40 & 6.248 & 0.067 & 0.827 & 12.497 & 0.143 & 1.534 & 24.994 & 0.230 & 2.829 \\
\hline 0.50 & 6.359 & 0.066 & 1.124 & 12.717 & 0.141 & 2.133 & 25.434 & 0.235 & 2.630 \\
\hline 0.60 & 6.820 & 0.069 & 1.103 & 13.640 & 0.133 & 1.881 & 27.280 & 0.235 & 2.406 \\
\hline 0.70 & 6.916 & 0.074 & 1.110 & 13.832 & 0.132 & 1.860 & 27.665 & 0.246 & 3.926 \\
\hline 0.80 & 5.770 & 0.068 & 1.230 & 11.539 & 0.112 & 2.734 & 23.079 & 0.244 & 4.674 \\
\hline 0.90 & 6.212 & 0.063 & 1.413 & 12.425 & 0.114 & 2.467 & 24.850 & 0.235 & 4.631 \\
\hline 1.00 & 4.959 & 0.055 & 1.996 & 9.917 & 0.094 & 2.689 & 19.835 & 0.240 & 5.294 \\
\hline 1.10 & 4.791 & 0.036 & 1.793 & 9.581 & 0.098 & 3.375 & 19.163 & 0.240 & 6.131 \\
\hline 1.20 & 3.921 & 0.041 & 1.891 & 7.843 & 0.086 & 3.844 & 15.685 & 0.229 & 6.047 \\
\hline 1.30 & 3.360 & 0.032 & 1.993 & 6.720 & 0.095 & 3.943 & 13.440 & 0.231 & 5.926 \\
\hline 1.40 & 3.543 & 0.031 & 2.209 & 7.086 & 0.087 & 4.094 & 14.172 & 0.218 & 5.861 \\
\hline 1.50 & 3.013 & 0.051 & 2.290 & 6.026 & 0.098 & 4.423 & 12.051 & 0.216 & 6.114 \\
\hline 1.60 & 3.604 & 0.049 & 2.125 & 7.208 & 0.094 & 4.218 & 14.416 & 0.210 & 6.320 \\
\hline 1.70 & 3.110 & 0.057 & 1.908 & 6.220 & 0.091 & 3.757 & 12.439 & 0.204 & 6.177 \\
\hline 1.80 & 2.742 & 0.049 & 2.201 & 5.484 & 0.086 & 3.842 & 10.968 & 0.192 & 6.625 \\
\hline 1.90 & 3.181 & 0.048 & 2.302 & 6.363 & 0.078 & 4.099 & 12.726 & 0.186 & 6.471 \\
\hline 2.00 & 3.164 & 0.050 & 2.260 & 6.329 & 0.090 & 3.535 & 12.658 & 0.176 & 5.495 \\
\hline
\end{tabular}

Table 5.5: Influence of earthquake intensity on RNC-a isolator performance, $\mathrm{m}$-sec units.

\begin{tabular}{|c|c|c|c|c|c|c|c|c|c|}
\hline \multirow{3}{*}{$T_{\mathrm{S}}$} & \multicolumn{3}{|c|}{ Kobe $0.5 g$} & \multicolumn{3}{|c|}{ Northridge $0.5 g$} & \multicolumn{3}{|c|}{ Parkfield $0.5 g$} \\
\hline & \multirow{2}{*}{$\begin{array}{r}\text { Fixed } \\
\text { Top } \\
\text { Accel. }\end{array}$} & \multicolumn{2}{|c|}{ Isolated } & \multirow{2}{*}{$\begin{array}{r}\text { Fixed } \\
\text { Top } \\
\text { Accel. }\end{array}$} & \multicolumn{2}{|c|}{ Isolated } & \multirow{2}{*}{$\begin{array}{r}\text { Fixed } \\
\text { Top } \\
\text { Accel. }\end{array}$} & \multicolumn{2}{|c|}{ Isolated } \\
\hline & & $\begin{array}{l}\text { Base } \\
\text { Disp. }\end{array}$ & $\begin{array}{r}\text { Top } \\
\text { Accel. }\end{array}$ & & $\begin{array}{c}\text { Base } \\
\text { Disp. }\end{array}$ & $\begin{array}{r}\text { Top } \\
\text { Accel. }\end{array}$ & & $\begin{array}{l}\text { Base } \\
\text { Disp. }\end{array}$ & $\begin{array}{r}\text { Top } \\
\text { Accel. }\end{array}$ \\
\hline 0.20 & 21.986 & 0.346 & 0.952 & 23.260 & 0.071 & 0.517 & 16.689 & 0.082 & 0.699 \\
\hline 0.30 & 24.225 & 0.346 & 1.231 & 12.698 & 0.072 & 0.722 & 12.220 & 0.082 & 0.971 \\
\hline 0.40 & 21.758 & 0.347 & 1.848 & 11.150 & 0.074 & 1.374 & 10.809 & 0.084 & 1.202 \\
\hline 0.50 & 11.834 & 0.341 & 2.097 & 8.426 & 0.075 & 1.685 & 11.060 & 0.087 & 1.490 \\
\hline 0.60 & 14.015 & 0.344 & 2.459 & 9.696 & 0.079 & 1.747 & 9.074 & 0.085 & 1.750 \\
\hline 0.70 & 14.224 & 0.353 & 3.113 & 11.928 & 0.080 & 1.918 & 8.973 & 0.077 & 1.928 \\
\hline 0.80 & 13.975 & 0.335 & 3.085 & 11.711 & 0.075 & 2.453 & 10.963 & 0.079 & 2.594 \\
\hline 0.90 & 20.544 & 0.322 & 4.390 & 7.233 & 0.087 & 2.431 & 9.434 & 0.072 & 2.383 \\
\hline 1.00 & 18.153 & 0.323 & 3.517 & 9.225 & 0.098 & 1.977 & 8.291 & 0.075 & 2.403 \\
\hline 1.10 & 24.315 & 0.336 & 3.759 & 9.602 & 0.093 & 2.647 & 10.912 & 0.057 & 2.336 \\
\hline 1.20 & 24.545 & 0.348 & 3.652 & 8.705 & 0.097 & 2.550 & 7.992 & 0.049 & 2.364 \\
\hline 1.30 & 20.874 & 0.358 & 5.298 & 6.725 & 0.106 & 3.571 & 6.905 & 0.062 & 2.131 \\
\hline 1.40 & 14.557 & 0.353 & 4.688 & 8.828 & 0.089 & 3.005 & 6.914 & 0.065 & 2.642 \\
\hline 1.50 & 11.423 & 0.302 & 5.092 & 5.899 & 0.094 & 3.707 & 6.423 & 0.066 & 2.572 \\
\hline 1.60 & 11.315 & 0.278 & 4.857 & 7.071 & 0.101 & 3.625 & 6.532 & 0.053 & 2.669 \\
\hline 1.70 & 11.120 & 0.278 & 4.466 & 7.482 & 0.096 & 4.268 & 6.197 & 0.064 & 3.133 \\
\hline 1.80 & 11.206 & 0.277 & 4.882 & 7.270 & 0.103 & 4.035 & 6.432 & 0.072 & 3.499 \\
\hline 1.90 & 13.795 & 0.273 & 6.555 & 8.319 & 0.106 & 5.203 & 5.114 & 0.083 & 3.486 \\
\hline 2.00 & 12.485 & 0.240 & 5.665 & 6.763 & 0.124 & 5.143 & 4.959 & 0.059 & 3.266 \\
\hline
\end{tabular}

Table 5.6: Influence of earthquake frequency contents on RNC-a isolator performance, m-sec units. 


\begin{tabular}{|c|c|c|c|c|c|c|c|c|c|c|c|c|c|c|c|c|}
\hline \multirow[b]{2}{*}{ No. } & \multirow[b]{2}{*}{ Earthquake Record } & \multicolumn{3}{|c|}{ Fixed-base } & \multicolumn{6}{|c|}{ Isolated by RNC type (a), set II } & \multicolumn{6}{|c|}{ Isolated by RNC type (b), set II } \\
\hline & & $\begin{array}{r}\text { Building } \\
\text { Drift } \\
\end{array}$ & $\begin{array}{r}\text { Top } \\
\text { Accel. }\end{array}$ & $\begin{array}{r}\text { Base } \\
\text { Shear }\end{array}$ & $\begin{array}{l}\text { Base } \\
\text { Disp. }\end{array}$ & $\begin{array}{r}\text { Building } \\
\text { Drift }\end{array}$ & $\begin{array}{r}\text { Top } \\
\text { Accel. }\end{array}$ & $\begin{array}{r}\text { Red. }^{a} \\
(\%)\end{array}$ & $\begin{array}{r}\text { Base } \\
\text { Shear }\end{array}$ & $\begin{array}{r}\text { Red. } \\
(\%)\end{array}$ & $\begin{array}{l}\text { Base } \\
\text { Disp. }\end{array}$ & $\begin{array}{r}\text { Building } \\
\text { Drift }\end{array}$ & $\begin{array}{r}\text { Top } \\
\text { Accel. }\end{array}$ & $\begin{array}{l}\text { Red. } \\
(\%)\end{array}$ & $\begin{array}{r}\text { Base } \\
\text { Shear }\end{array}$ & $\begin{array}{r}\text { Red. } \\
(\%)\end{array}$ \\
\hline 1 & ALTADENA $0^{\circ}$ & 0.087 & 17.36 & 1837.13 & 0.03 & 0.003 & 2.03 & $88 \%$ & 122.35 & $93 \%$ & 0.05 & 0.006 & 3.32 & $81 \%$ & 249.25 & $86 \%$ \\
\hline 2 & ALTADENA $90^{\circ}$ & 0.046 & 8.95 & 1061.07 & 0.01 & 0.003 & 0.67 & $93 \%$ & 102.76 & $90 \%$ & 0.01 & 0.004 & 1.47 & $84 \%$ & 123.03 & $88 \%$ \\
\hline 3 & ARRAY06 $0^{\circ}$ & 0.052 & 9.26 & 1299.02 & 0.34 & 0.006 & 1.69 & $82 \%$ & 263.46 & $80 \%$ & 0.23 & 0.023 & 3.99 & $57 \%$ & 769.67 & $41 \%$ \\
\hline 4 & ARRAY06 $90^{\circ}$ & 0.073 & 12.08 & 1853.28 & 0.99 & 0.014 & 2.23 & $82 \%$ & 409.70 & $78 \%$ & 0.30 & 0.027 & 5.10 & $58 \%$ & 966.25 & $48 \%$ \\
\hline 5 & CORRALIT $0^{\circ}$ & 0.125 & 21.35 & 2809.14 & 0.11 & 0.006 & 2.06 & $90 \%$ & 141.78 & $95 \%$ & 0.09 & 0.011 & 3.93 & $82 \%$ & 374.00 & $87 \%$ \\
\hline 6 & CORRALIT $90^{\circ}$ & 0.093 & 16.37 & 2512.62 & 0.14 & 0.006 & 2.27 & $86 \%$ & 148.12 & $94 \%$ & 0.06 & 0.010 & 3.42 & $79 \%$ & 421.89 & $83 \%$ \\
\hline 7 & HOLLISTE $0^{\circ}$ & 0.127 & 19.59 & 3159.75 & 0.29 & 0.004 & 1.96 & $90 \%$ & 199.81 & $94 \%$ & 0.26 & 0.026 & 5.23 & $73 \%$ & 841.45 & $73 \%$ \\
\hline 8 & HOLLISTE $90^{\circ}$ & 0.072 & 12.06 & 1721.99 & 0.11 & 0.004 & 1.32 & $89 \%$ & 137.28 & $92 \%$ & 0.05 & 0.007 & 2.37 & $80 \%$ & 282.42 & $84 \%$ \\
\hline 9 & LACC-NOR $0^{\circ}$ & 0.055 & 9.62 & 1263.41 & 0.07 & 0.004 & 1.82 & $81 \%$ & 124.81 & $90 \%$ & 0.06 & 0.012 & 2.53 & $74 \%$ & 274.41 & $78 \%$ \\
\hline 10 & LACC-NOR $90^{\circ}$ & 0.041 & 7.40 & 1087.29 & 0.10 & 0.005 & 1.28 & $83 \%$ & 134.00 & $88 \%$ & 0.04 & 0.007 & 2.53 & $66 \%$ & 227.48 & $79 \%$ \\
\hline 11 & LEXINGT $0^{\circ}$ & 0.075 & 12.21 & 1893.83 & 0.22 & 0.005 & 2.04 & $83 \%$ & 178.85 & $91 \%$ & 0.26 & 0.029 & 5.17 & $58 \%$ & 839.16 & $56 \%$ \\
\hline 12 & LEXINGT $90^{\circ}$ & 0.071 & 11.64 & 1719.58 & 0.38 & 0.007 & 1.46 & $87 \%$ & 226.87 & $87 \%$ & 0.30 & 0.033 & 5.81 & $50 \%$ & 964.05 & $44 \%$ \\
\hline 13 & LUCERNE $0^{\circ}$ & 0.034 & 13.99 & 1108.82 & 0.13 & 0.005 & 0.94 & $93 \%$ & 151.56 & $86 \%$ & 0.11 & 0.012 & 2.69 & $81 \%$ & 411.93 & $63 \%$ \\
\hline 14 & LUCERNE $90^{\circ}$ & 0.050 & 17.62 & 1395.45 & 0.06 & 0.003 & 1.90 & $89 \%$ & 130.45 & $91 \%$ & 0.03 & 0.008 & 2.95 & $83 \%$ & 233.30 & $83 \%$ \\
\hline 15 & NEW-HALL $0^{\circ}$ & 0.179 & 27.49 & 4400.92 & 0.47 & 0.009 & 2.66 & $90 \%$ & 256.62 & $94 \%$ & 0.34 & 0.033 & 5.25 & $81 \%$ & 1087.43 & $75 \%$ \\
\hline 16 & NEW-HALL $90^{\circ}$ & 0.118 & 20.74 & 3124.83 & 0.19 & 0.009 & 3.03 & $85 \%$ & 164.57 & $95 \%$ & 0.17 & 0.016 & 4.15 & $80 \%$ & 596.30 & $81 \%$ \\
\hline 17 & OAK-WHAF $0^{\circ}$ & 0.057 & 8.84 & 1465.21 & 0.18 & 0.004 & 1.67 & $81 \%$ & 161.49 & $89 \%$ & 0.12 & 0.018 & 3.53 & $60 \%$ & 454.82 & $69 \%$ \\
\hline 18 & OAK-WHAF $90^{\circ}$ & 0.052 & 7.44 & 1458.03 & 0.08 & 0.004 & 1.37 & $82 \%$ & 133.08 & $91 \%$ & 0.14 & 0.018 & 4.11 & $45 \%$ & 507.79 & $65 \%$ \\
\hline 19 & PETROLIA $0^{\circ}$ & 0.124 & 20.01 & 2934.93 & 0.05 & 0.006 & 2.84 & $86 \%$ & 156.74 & $95 \%$ & 0.07 & 0.018 & 4.50 & $78 \%$ & 440.22 & $85 \%$ \\
\hline 20 & PETROLIA $90^{\circ}$ & 0.125 & 21.10 & 3379.69 & 0.53 & 0.008 & 2.60 & $88 \%$ & 268.81 & $92 \%$ & 0.28 & 0.029 & 5.01 & $76 \%$ & 921.44 & $73 \%$ \\
\hline 21 & POMONA $0^{\circ}$ & 0.019 & 4.57 & 445.67 & 0.03 & 0.004 & 0.83 & $82 \%$ & 112.72 & $75 \%$ & 0.02 & 0.006 & 2.49 & $46 \%$ & 146.52 & $67 \%$ \\
\hline 22 & POMONA $90^{\circ}$ & 0.014 & 4.70 & 343.51 & 0.02 & 0.004 & 0.70 & $85 \%$ & 110.44 & $68 \%$ & 0.01 & 0.004 & 2.14 & $54 \%$ & 153.10 & $55 \%$ \\
\hline 23 & SANTA-MONICA $0^{\circ}$ & 0.051 & 9.70 & 1100.61 & 0.04 & 0.005 & 0.90 & $91 \%$ & 130.95 & $88 \%$ & 0.05 & 0.008 & 2.74 & $72 \%$ & 238.84 & $78 \%$ \\
\hline 24 & SANTA-MONICA $90^{\circ}$ & 0.071 & 14.88 & 1616.43 & 0.11 & 0.006 & 2.99 & $80 \%$ & 165.01 & $90 \%$ & 0.14 & 0.014 & 6.22 & $58 \%$ & 500.77 & $69 \%$ \\
\hline 25 & SYLMAR $0^{\circ}$ & 0.185 & 29.60 & 4216.62 & 0.48 & 0.006 & 2.63 & $91 \%$ & 265.88 & $94 \%$ & 0.53 & 0.055 & 8.32 & $72 \%$ & 1603.14 & $62 \%$ \\
\hline 26 & SYLMAR $90^{\circ}$ & 0.144 & 22.05 & 3739.71 & 0.26 & 0.008 & 2.30 & $90 \%$ & 192.69 & $95 \%$ & 0.29 & 0.033 & 6.08 & $72 \%$ & 929.76 & $75 \%$ \\
\hline 27 & YERMO $0^{\circ}$ & 0.056 & 8.92 & 1365.22 & 0.08 & 0.004 & 1.01 & $89 \%$ & 142.86 & $90 \%$ & 0.06 & 0.010 & 2.31 & $74 \%$ & 315.81 & $77 \%$ \\
\hline 28 & YERMO $90^{\circ}$ & 0.060 & 10.10 & 1518.83 & 0.20 & 0.003 & 1.64 & $84 \%$ & 162.34 & $89 \%$ & 0.17 & 0.020 & 3.62 & $64 \%$ & 579.72 & $62 \%$ \\
\hline 29 & EL-CENTRO & 0.080 & 12.72 & 2054.51 & 0.14 & 0.004 & 2.13 & $83 \%$ & 148.88 & $93 \%$ & 0.06 & 0.011 & 3.09 & $76 \%$ & 305.50 & $85 \%$ \\
\hline 30 & KERN & 0.029 & 5.17 & 809.78 & 0.04 & 0.005 & 1.06 & $80 \%$ & 116.31 & $86 \%$ & 0.04 & 0.009 & 1.81 & $65 \%$ & 216.14 & $73 \%$ \\
\hline 31 & KOBE & 0.101 & 16.07 & 2640.23 & 0.32 & 0.008 & 2.82 & $82 \%$ & 252.72 & $90 \%$ & 0.70 & 0.067 & 9.55 & $41 \%$ & 2118.55 & $20 \%$ \\
\hline 32 & LOMA-PRIETA & 0.053 & 8.35 & 1367.53 & 0.08 & 0.005 & 1.51 & $82 \%$ & 130.73 & $90 \%$ & 0.10 & 0.014 & 4.03 & $52 \%$ & 382.35 & $72 \%$ \\
\hline 33 & MEXICO & 0.013 & 1.88 & 355.02 & 0.20 & 0.006 & 1.20 & $36 \%$ & 165.35 & $53 \%$ & 0.20 & 0.022 & 3.48 & $-85 \%$ & 680.11 & $-92 \%$ \\
\hline 34 & NORTHRIDGE & 0.071 & 14.88 & 1616.55 & 0.11 & 0.006 & 2.99 & $80 \%$ & 165.02 & $90 \%$ & 0.14 & 0.014 & 6.22 & $58 \%$ & 500.84 & $69 \%$ \\
\hline 35 & PARKFIELD & 0.027 & 5.24 & 625.93 & 0.05 & 0.003 & 0.95 & $82 \%$ & 116.20 & $81 \%$ & 0.02 & 0.006 & 1.31 & $75 \%$ & 149.42 & $76 \%$ \\
\hline 36 & SAN-FERNANDO & 0.169 & 37.48 & 3772.02 & 0.51 & 0.007 & 2.18 & $94 \%$ & 271.16 & $93 \%$ & 0.44 & 0.044 & 8.50 & $77 \%$ & 1348.62 & $64 \%$ \\
\hline
\end{tabular}

Table 5.7: Results summary using 36 earthquakes and two RNC types: $a$ and b, ton-m-sec units.

${ }^{a}$ Reduction as a percentage of the corresponding fixed-base response 